\title{
Iron-catalyzed synthesis of sulfur-containing heterocycles
}

Cyril Bosset, ${ }^{\mathrm{a}}$ Gauthier Lefebvre, ${ }^{\mathrm{a}}$ Patrick Angibaud, ${ }^{\mathrm{b}}$ Ian Stansfield, ${ }^{\mathrm{b}}$ Lieven Meerpoel, ${ }^{\mathrm{c}}$ Didier Berthelot, ${ }^{\mathrm{b}}$ Amandine Guérinot ${ }^{* \mathrm{a}}$ and Janine Cossy* ${ }^{\mathrm{a}}$.

${ }^{a}$ Laboratoire de Chimie Organique, Institute of Chemistry, Biology and Innovation (CBI)-

UMR 8231, ESPCI Paris, CNRS, PSL Research University,10 rue Vauquelin 75231 Paris

Cedex 05, France.

${ }^{\mathrm{b} J a n s s e n ~ R e s e a r c h ~ \& ~ D e v e l o p m e n t, ~ O n c o l o g y ~ D i s c o v e r y ~ C h e m i s t r y, ~ C a m p u s ~ d e ~ M a i g r e m o n t ~}$ CS 10615, 27106, Val de Reuil Cedex, France.

${ }^{\mathrm{c} J a n s s e n ~ R e s e a r c h ~ \& ~ D e v e l o p m e n t, ~ J a n s s e n ~ P h a r m a c e u t i c a ~ N V ~ T u r n h o u t s e w e g ~ 30, ~} 2340$ Beerse, Belgium.

janine.cossy@espci.fr; amandine.guerinot@espci.fr

tel: 33140794429 

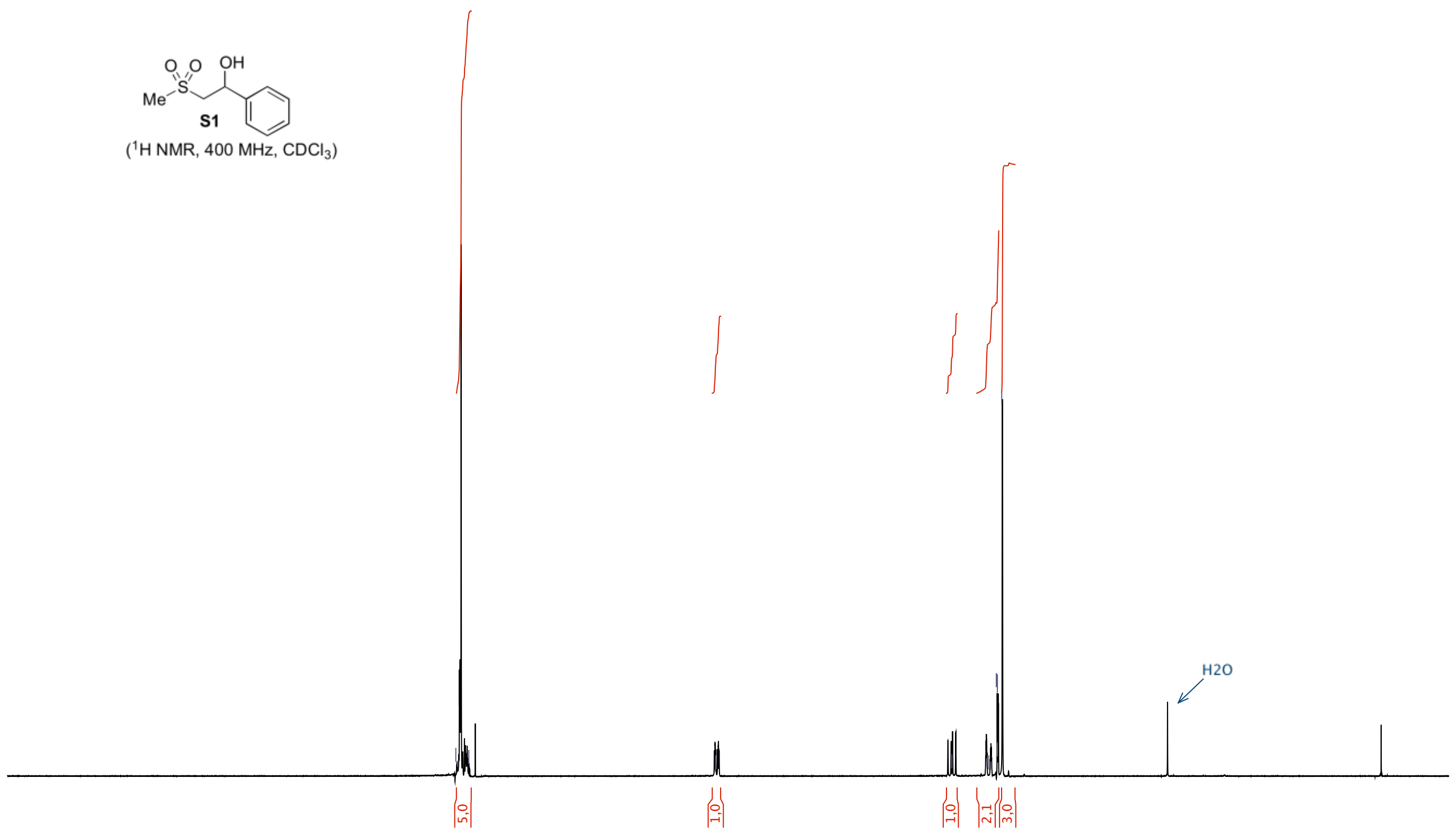
$\frac{\varepsilon}{2}$

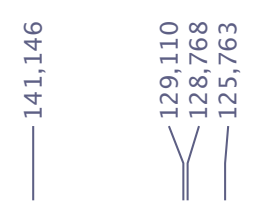
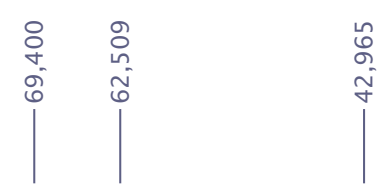<smiles>CS(=O)(=O)CC(O)c1ccccc1</smiles>

$\left({ }^{13} \mathrm{C} \mathrm{NMR}, 100 \mathrm{MHz}, \mathrm{CDCl}_{3}\right)$
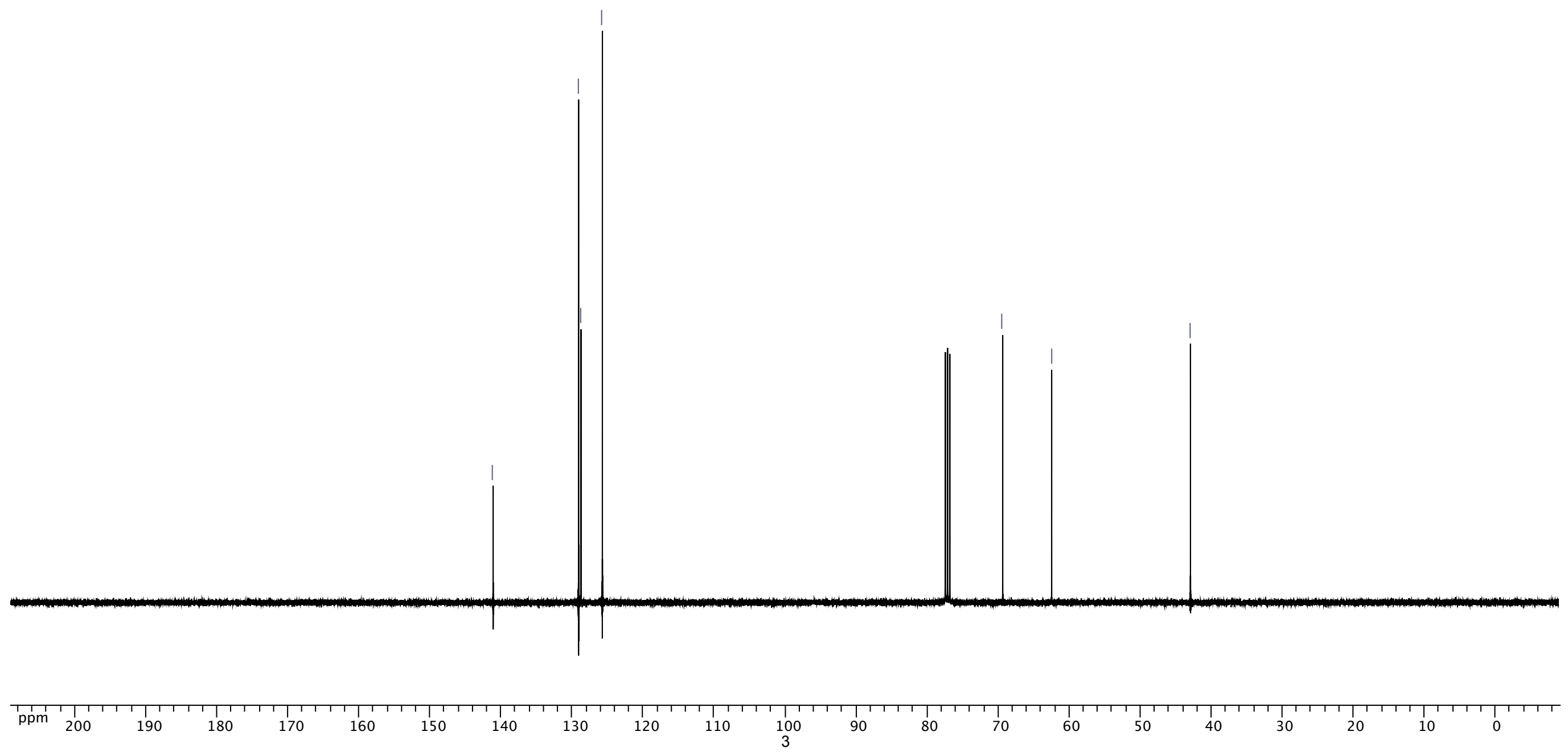


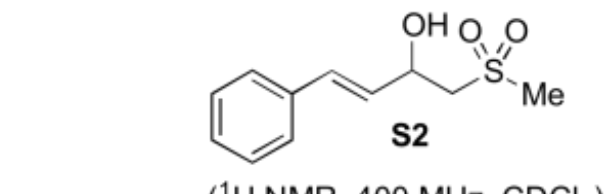

( ${ }^{1} \mathrm{H} \mathrm{NMR}, 400 \mathrm{MHz}, \mathrm{CDCl}_{3}$ )

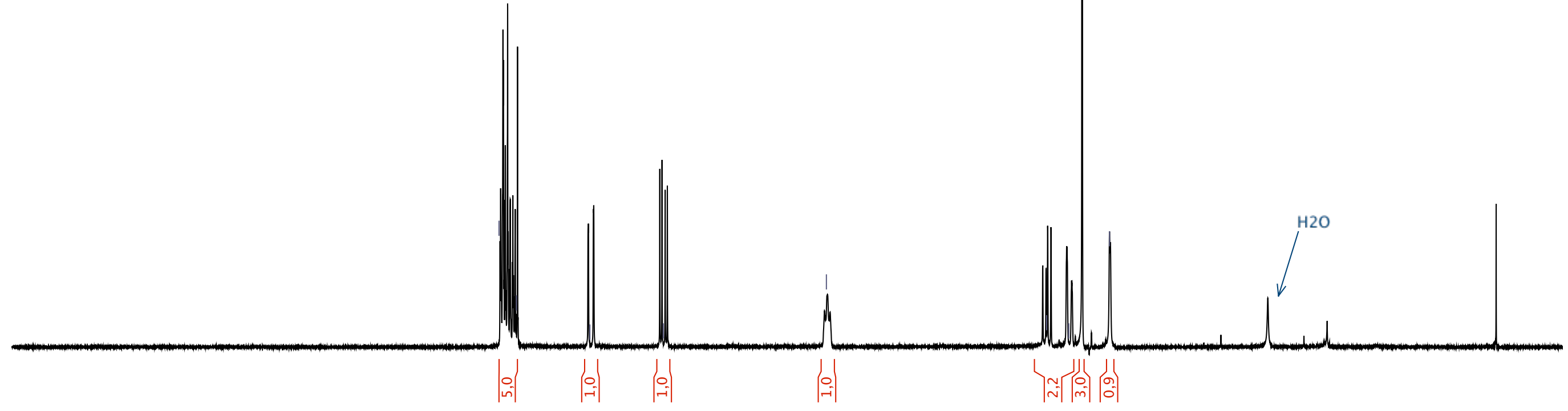


$\frac{\varepsilon}{2}$
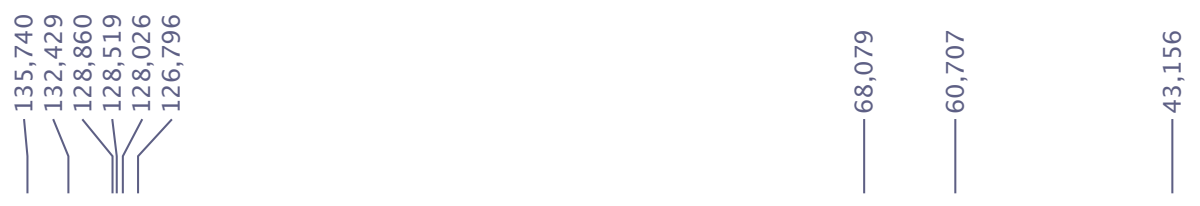<smiles>CS(=O)(=O)CC(O)/C=C/c1ccccc1</smiles>

$\left({ }^{13} \mathrm{C} \mathrm{NMR}, 100 \mathrm{MHz}, \mathrm{CDCl}_{3}\right)$
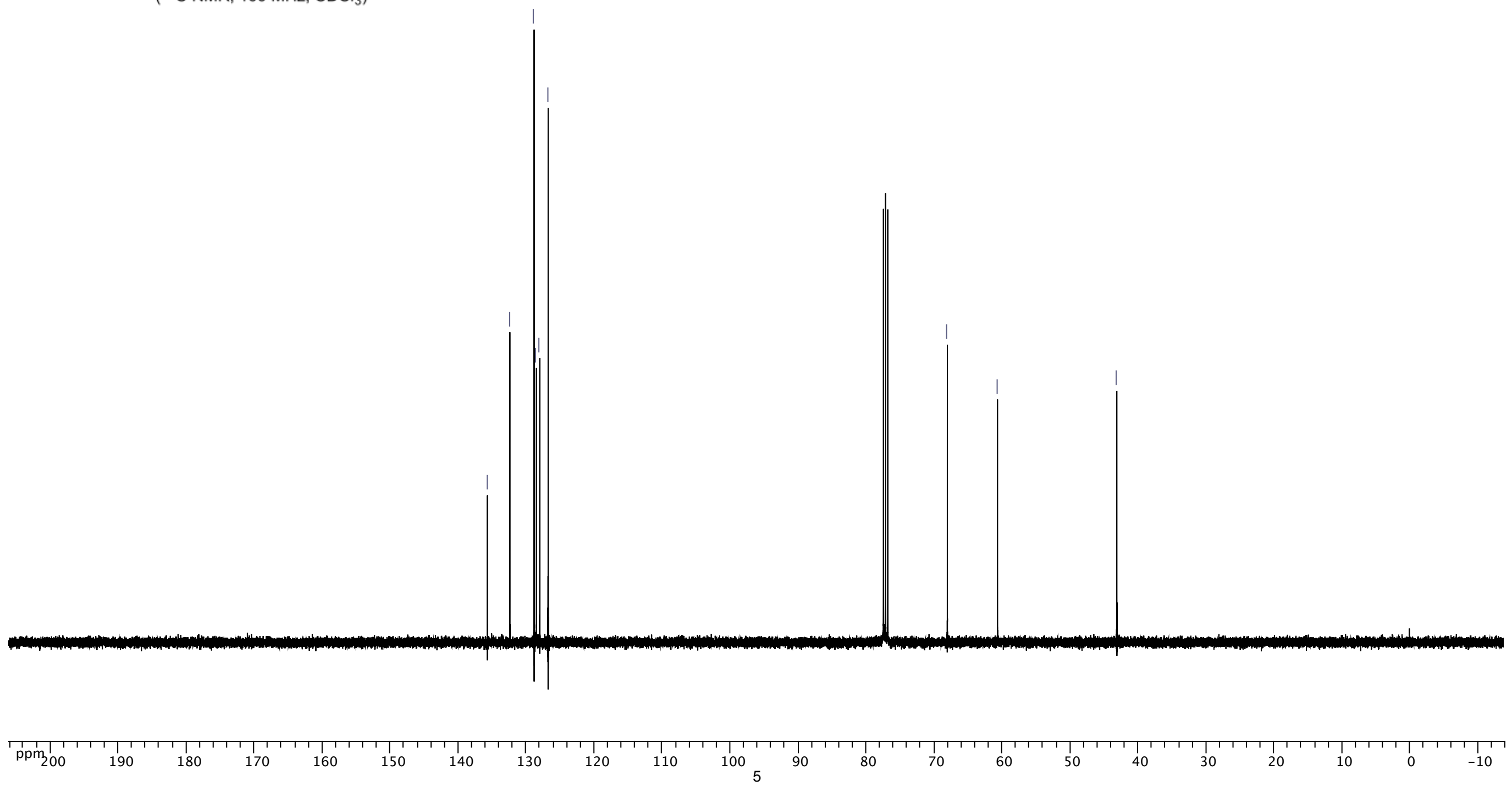


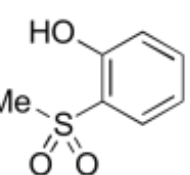

S3

$\left({ }^{1} \mathrm{H} \mathrm{NMR}, 400 \mathrm{MHz}, \mathrm{CDCl}_{3}\right.$ )
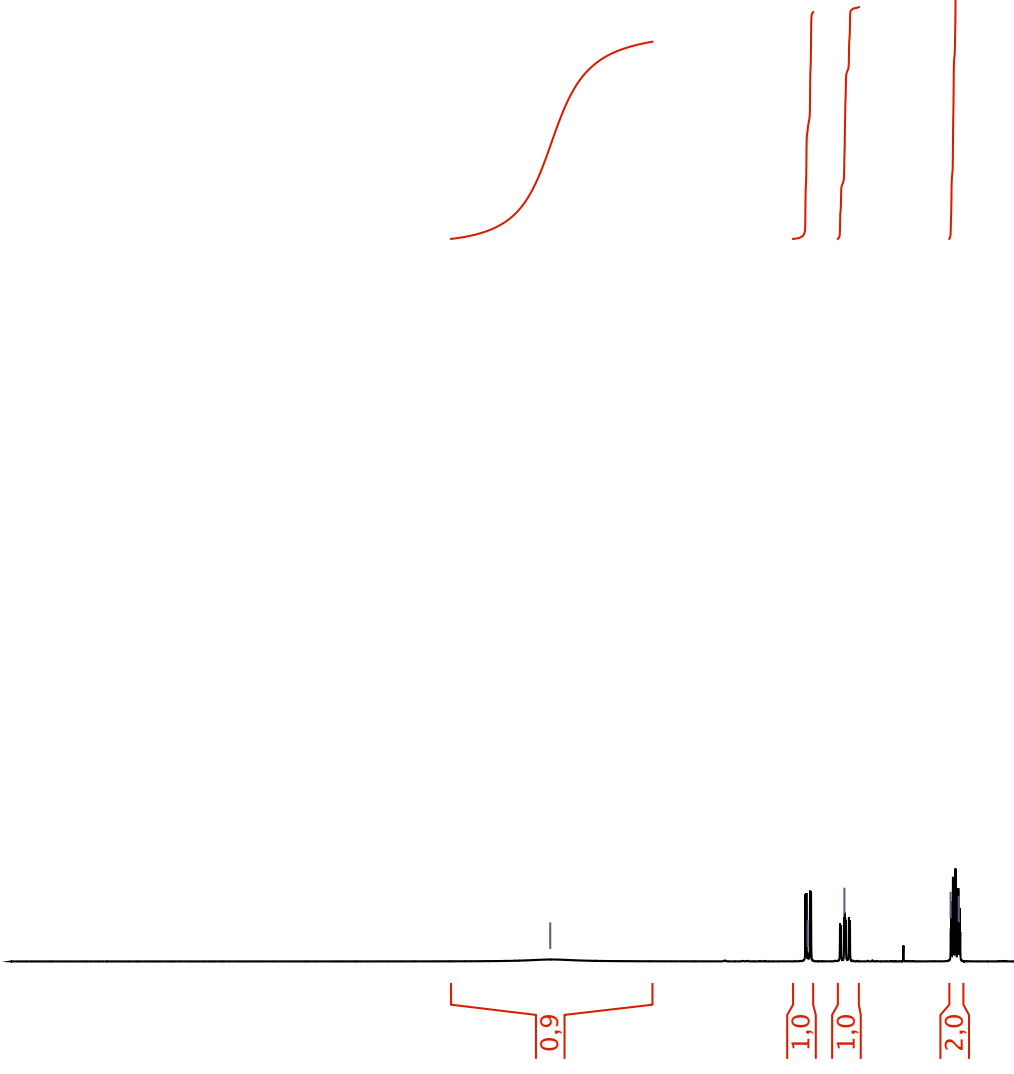


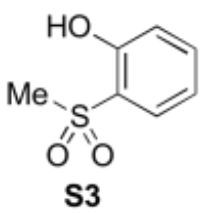

$\left({ }^{13} \mathrm{C}\right.$ NMR, $\left.100 \mathrm{MHz}, \mathrm{CDCl}_{3}\right)$

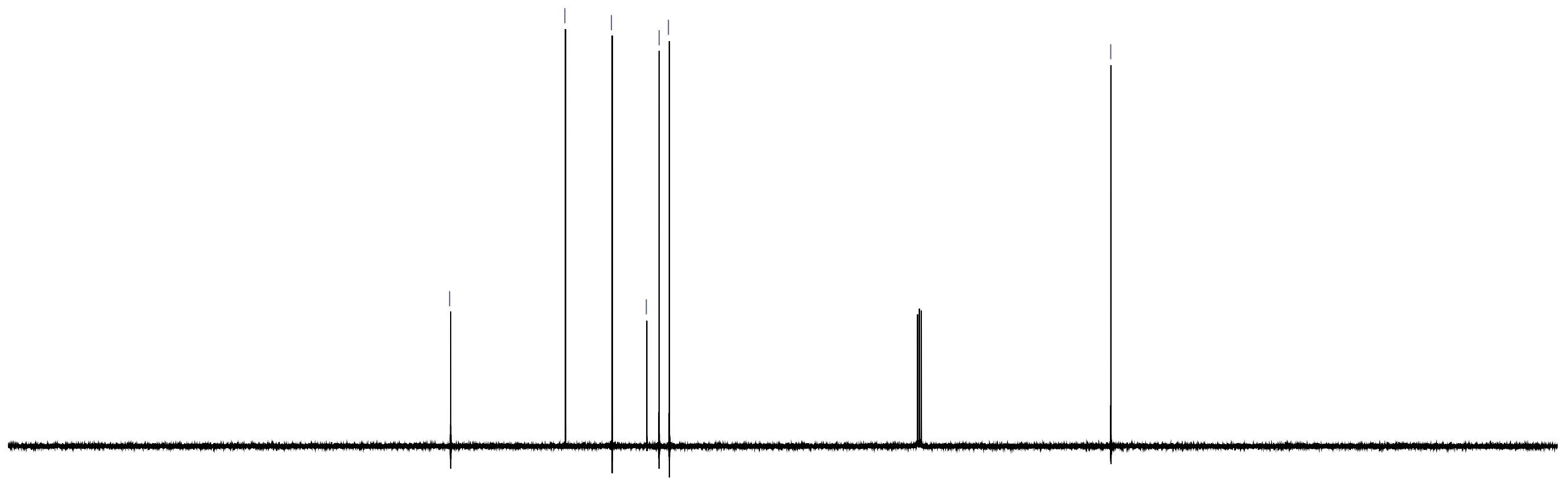




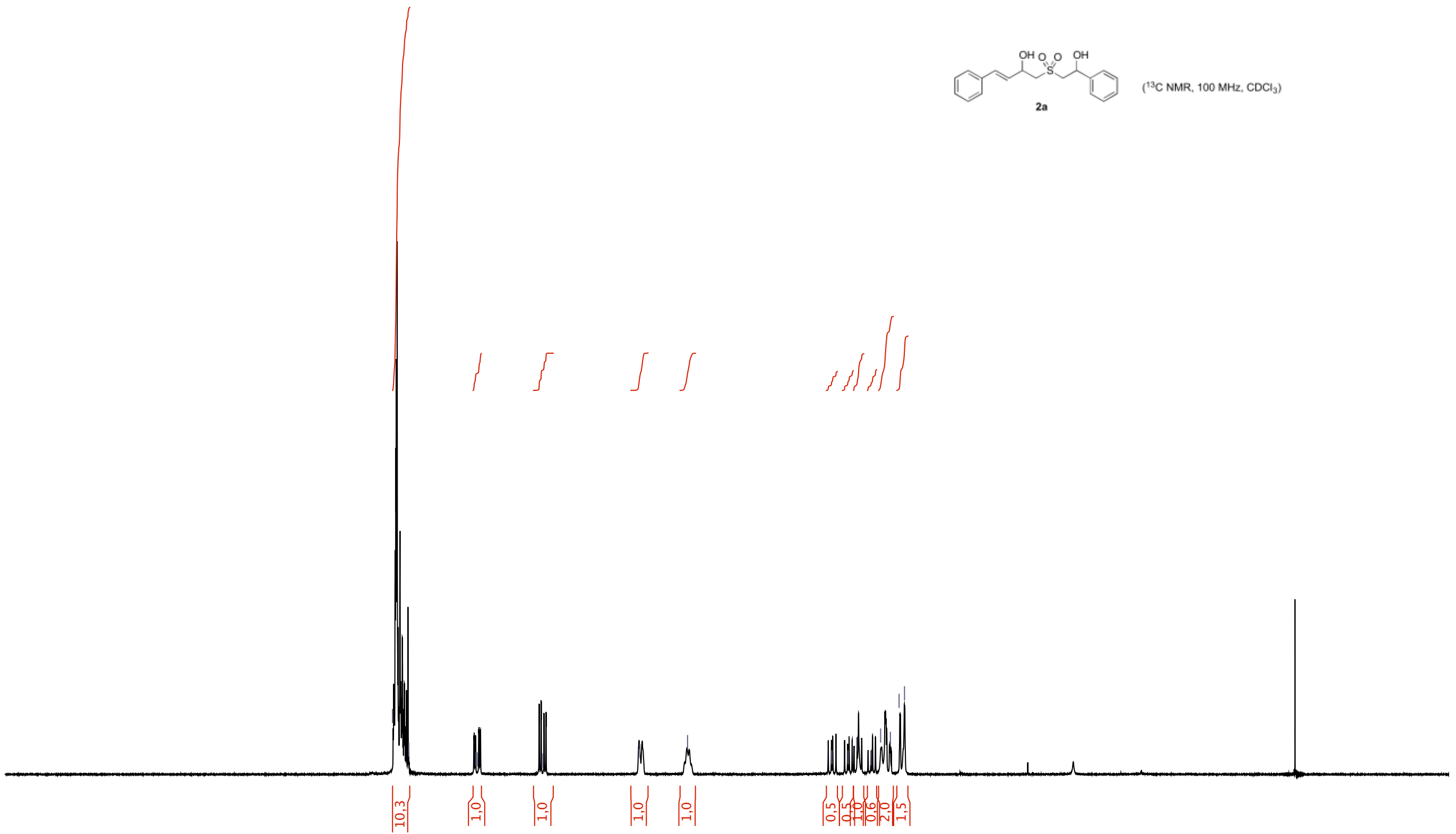




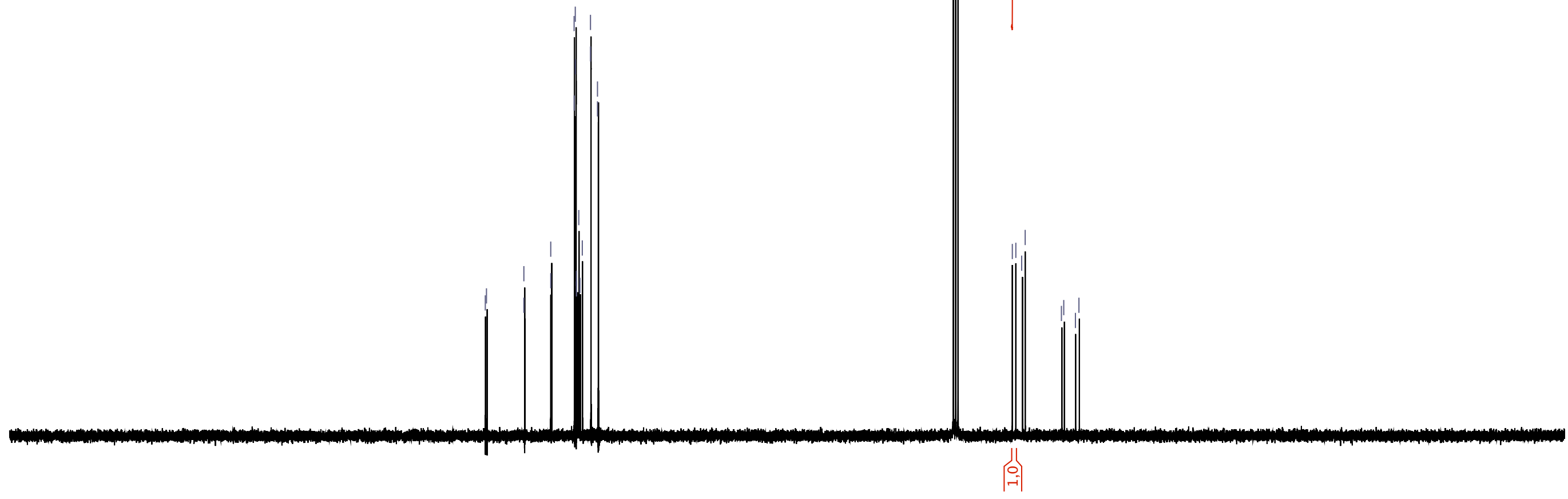




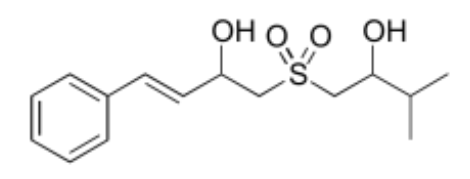

( ${ }^{1} \mathrm{H} \mathrm{NMR}, 400 \mathrm{MHz}, \mathrm{CDCl}_{3}$ )

2b

( $\mathrm{NMR}, 400 \mathrm{MHz}, \mathrm{CDCl}_{3}$ )
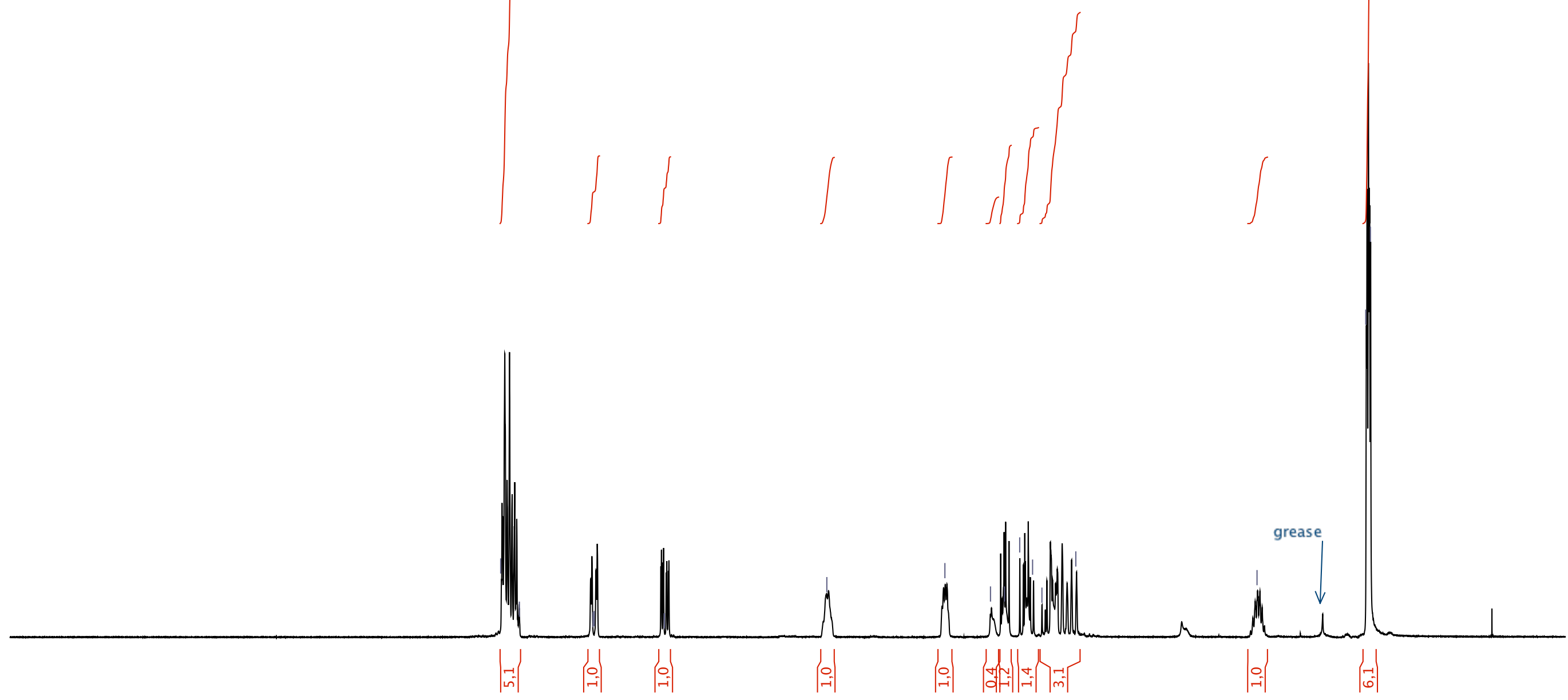
$\mathrm{OHOO} \mathrm{OH}$

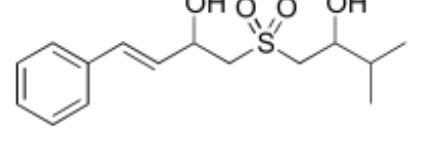

$\left({ }^{13} \mathrm{C} \mathrm{NMR}, 100 \mathrm{MHz}, \mathrm{CDCl}_{3}\right)$

2b

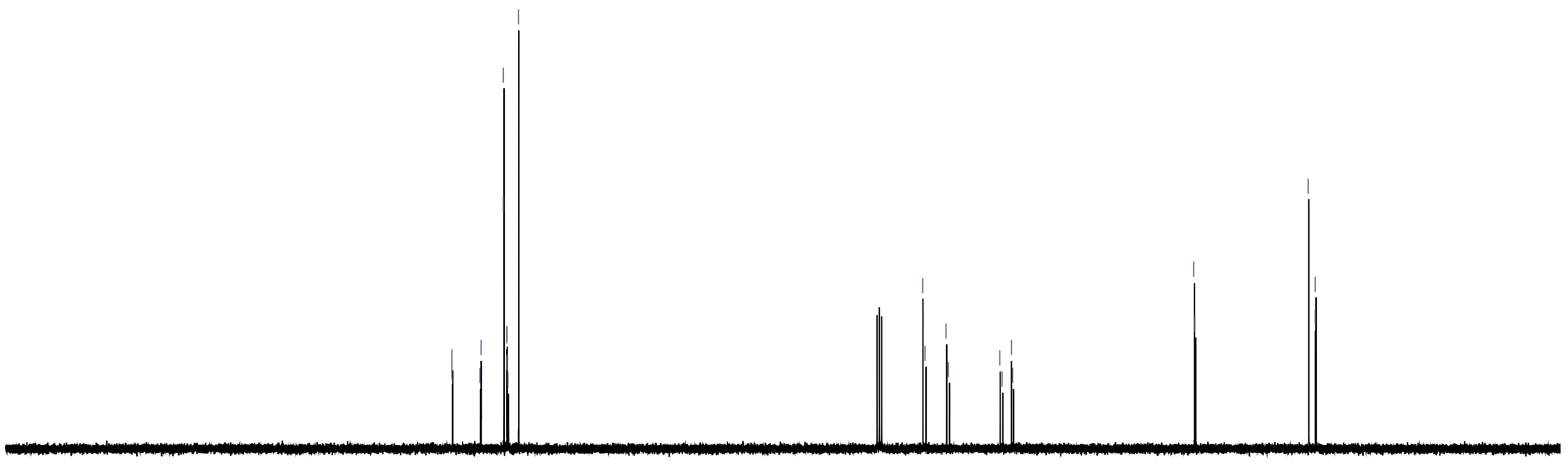



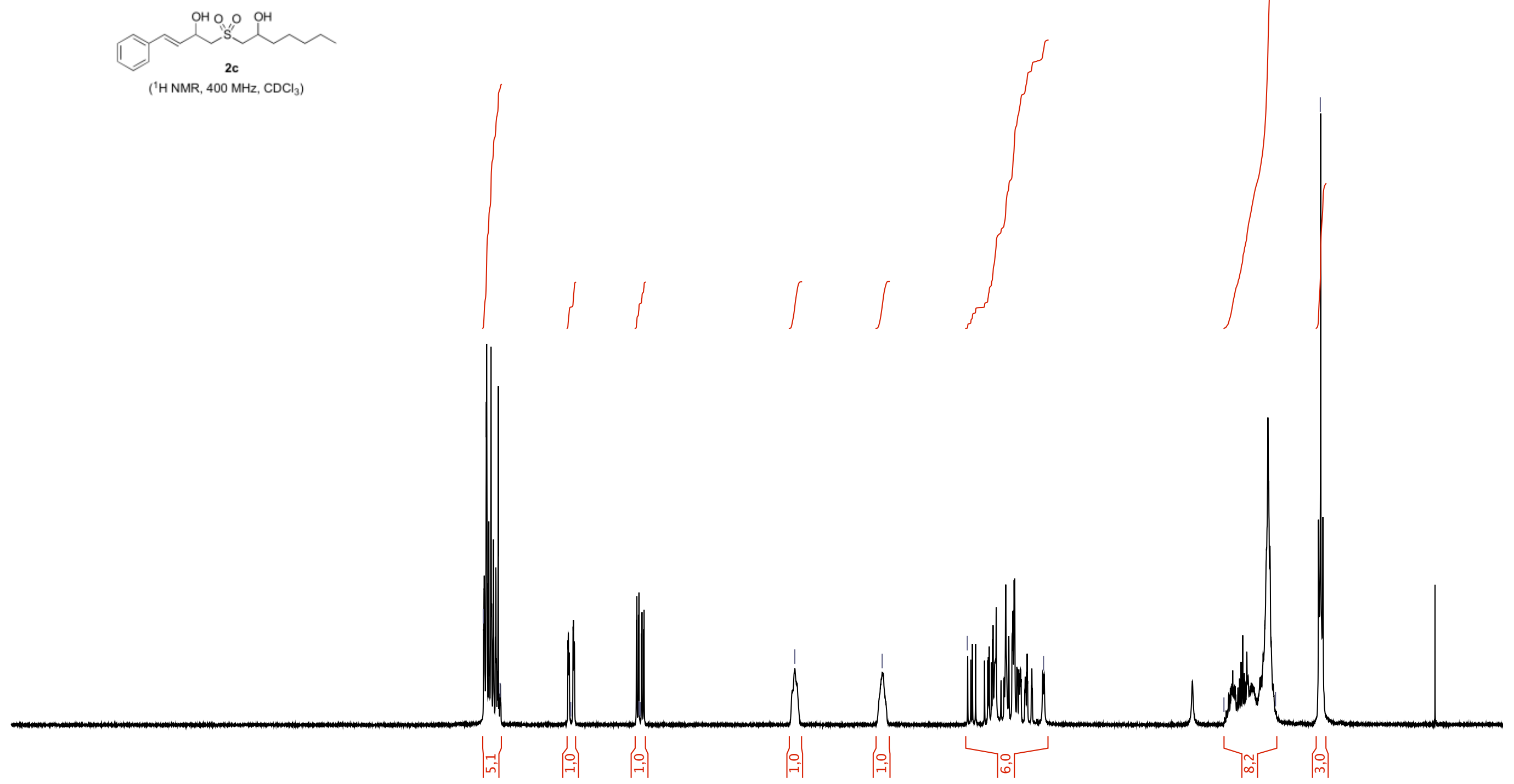
$\mathrm{OH} \mathrm{O} O \mathrm{OH}$

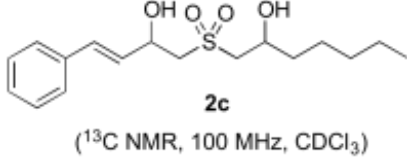

$\left({ }^{13} \mathrm{C}\right.$ NMR, $\left.100 \mathrm{MHz}, \mathrm{CDCl}_{3}\right)$

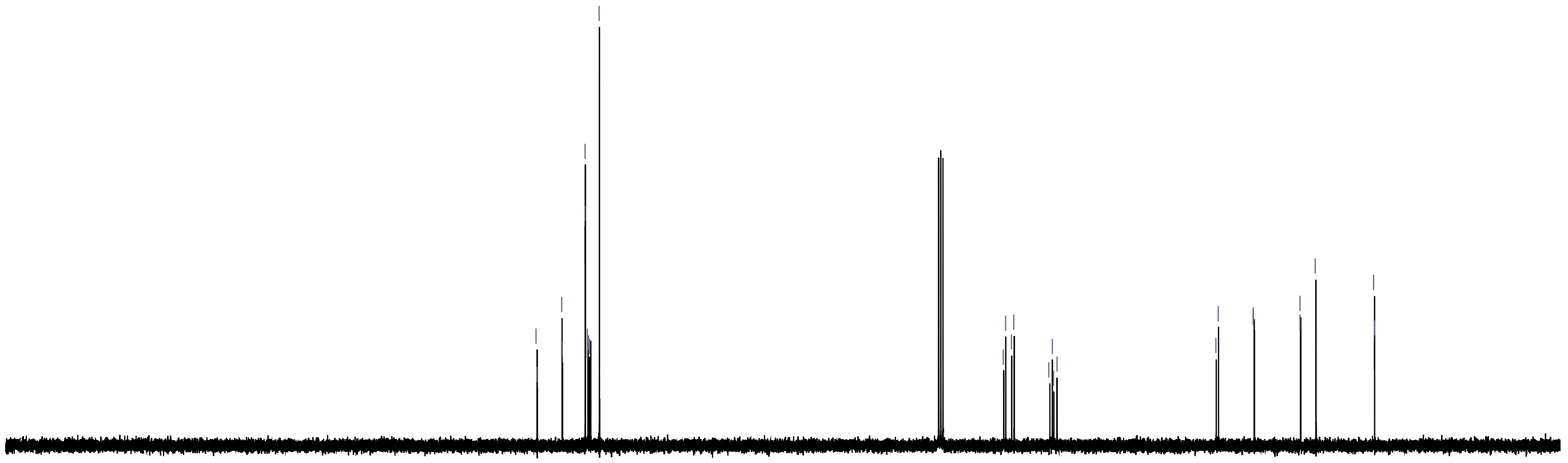



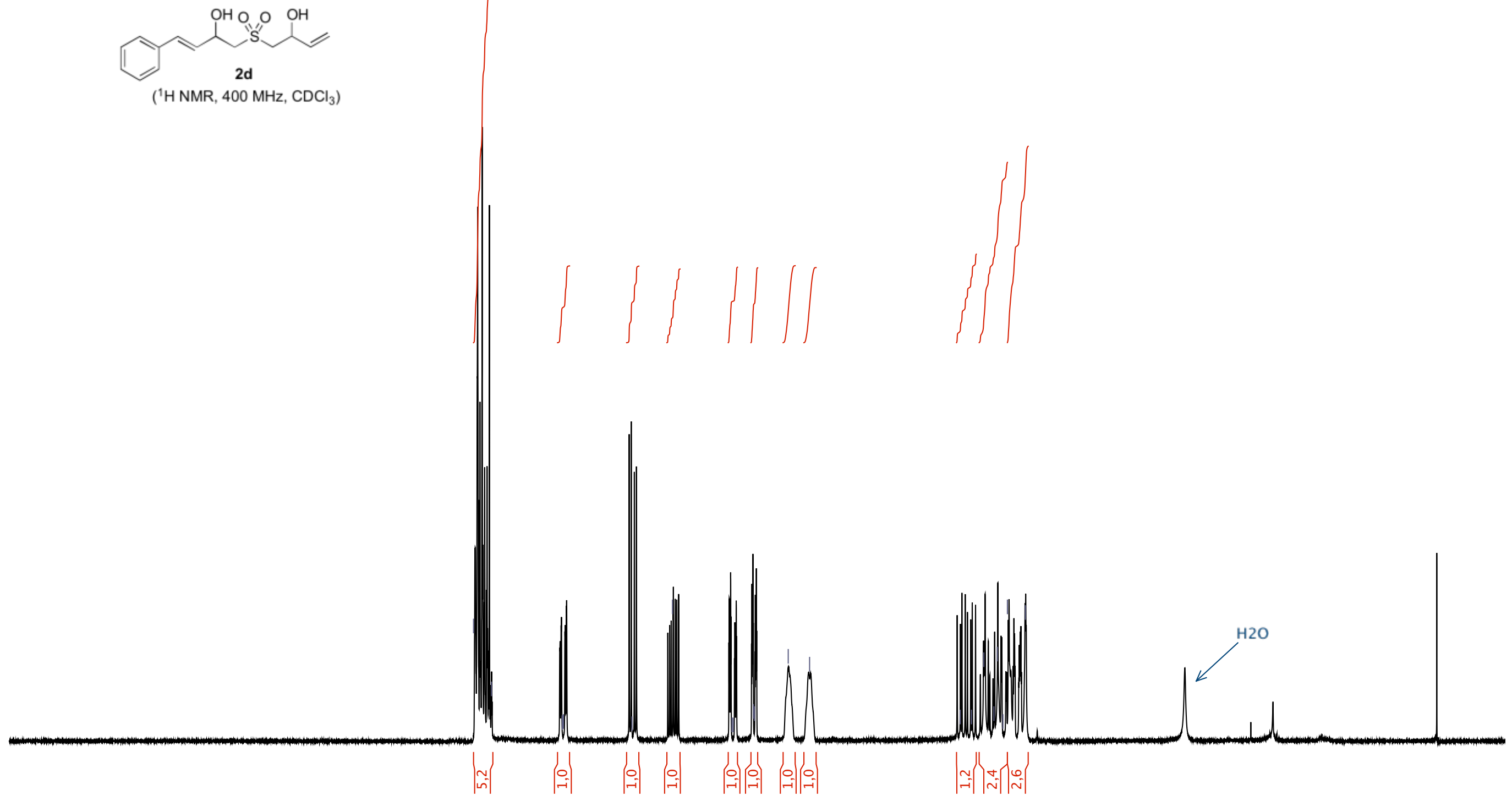


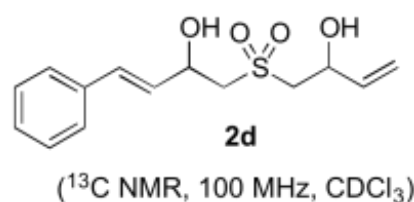

$\left({ }^{13} \mathrm{C} \mathrm{NMR}, 100 \mathrm{MHz}, \mathrm{CDCl}_{3}\right)$

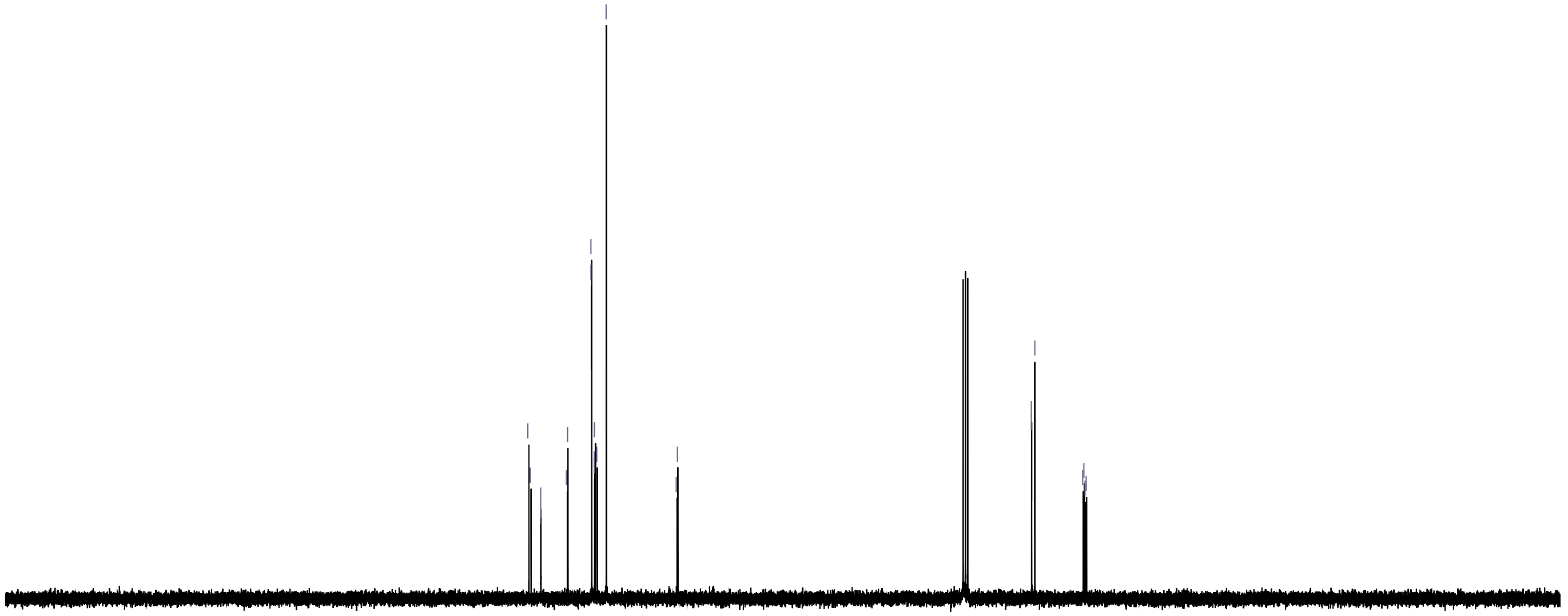


$\mathrm{OHOO} \mathrm{OH}$

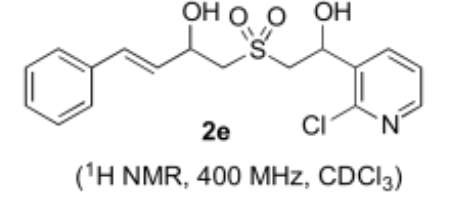

( ${ }^{1} \mathrm{H} \mathrm{NMR}, 400 \mathrm{MHz}, \mathrm{CDCl}_{3}$ )

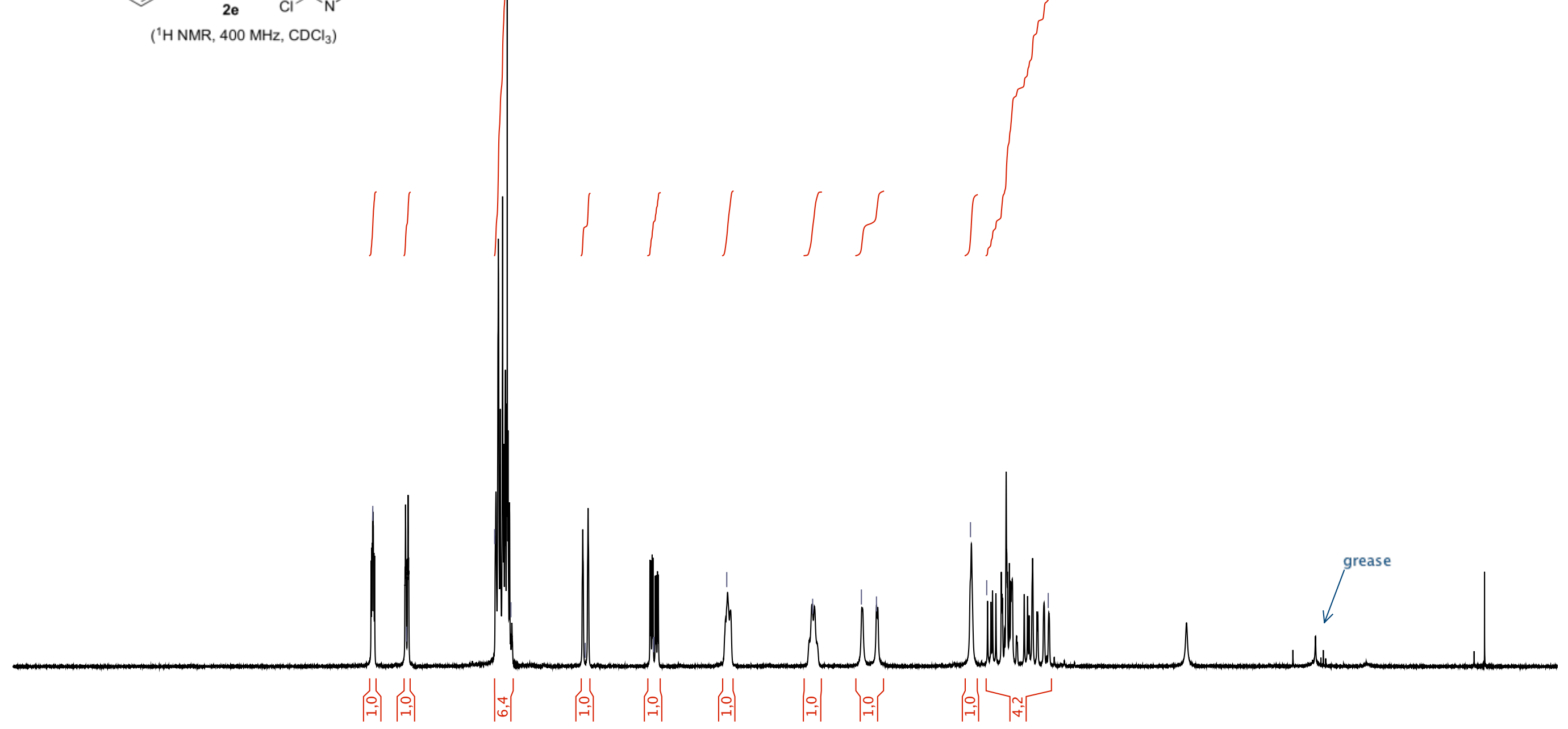


$\mathrm{OHO} \mathrm{OH}$

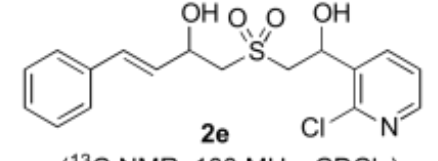

$\left({ }^{13} \mathrm{C} \mathrm{NMR}, 100 \mathrm{MHz}, \mathrm{CDCl}_{3}\right)$

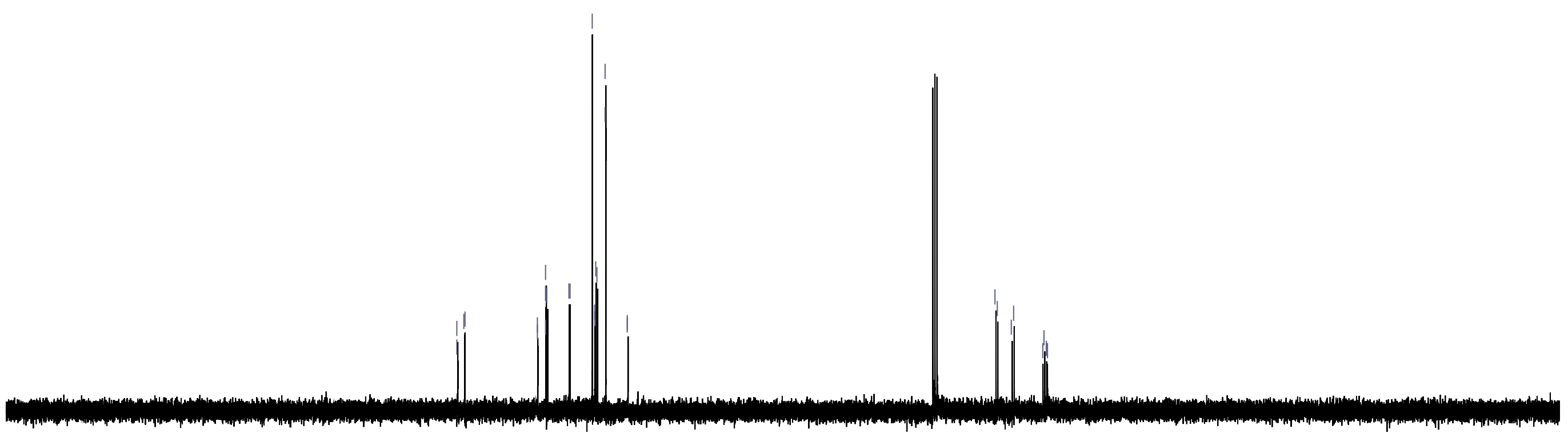




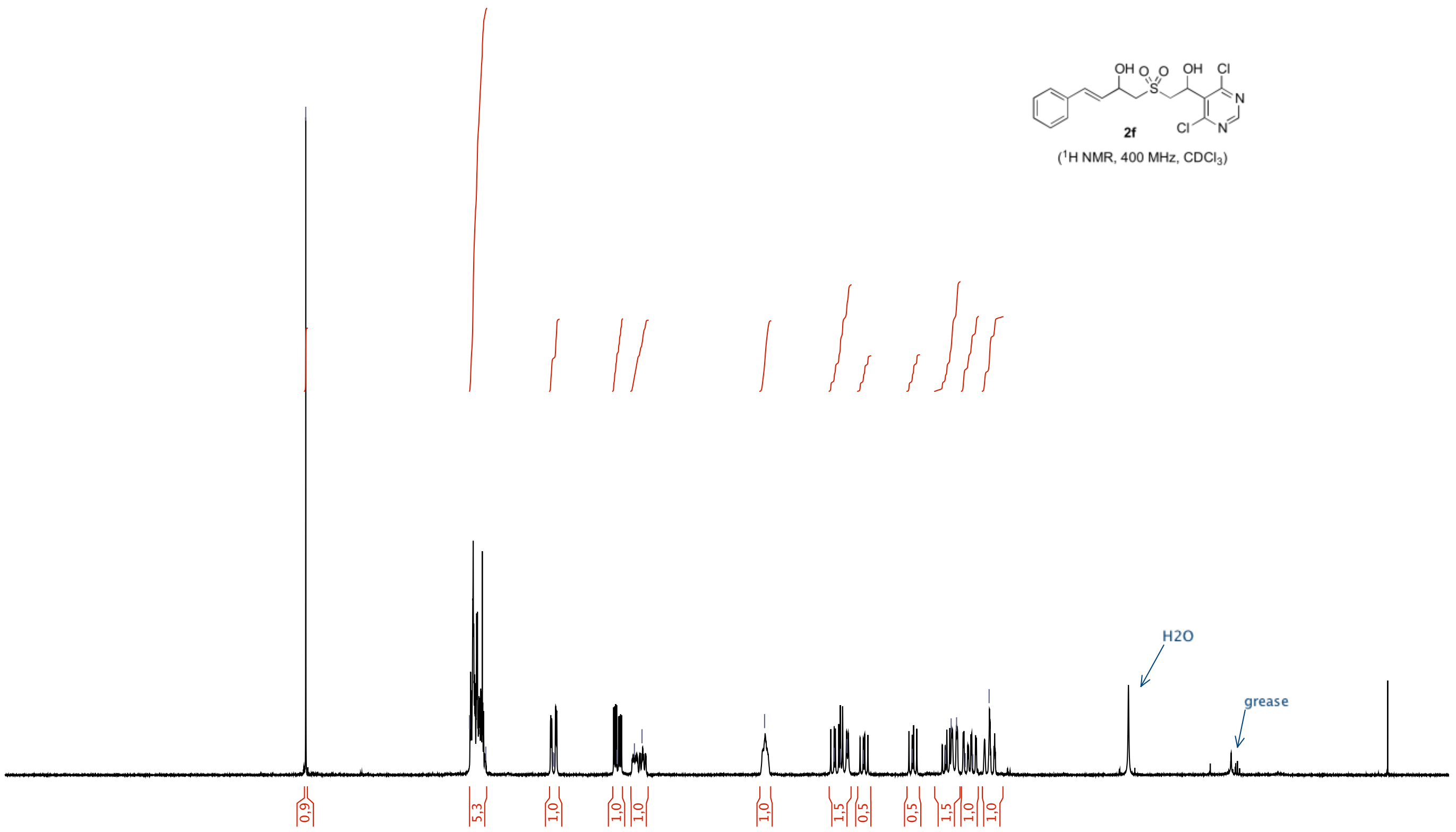



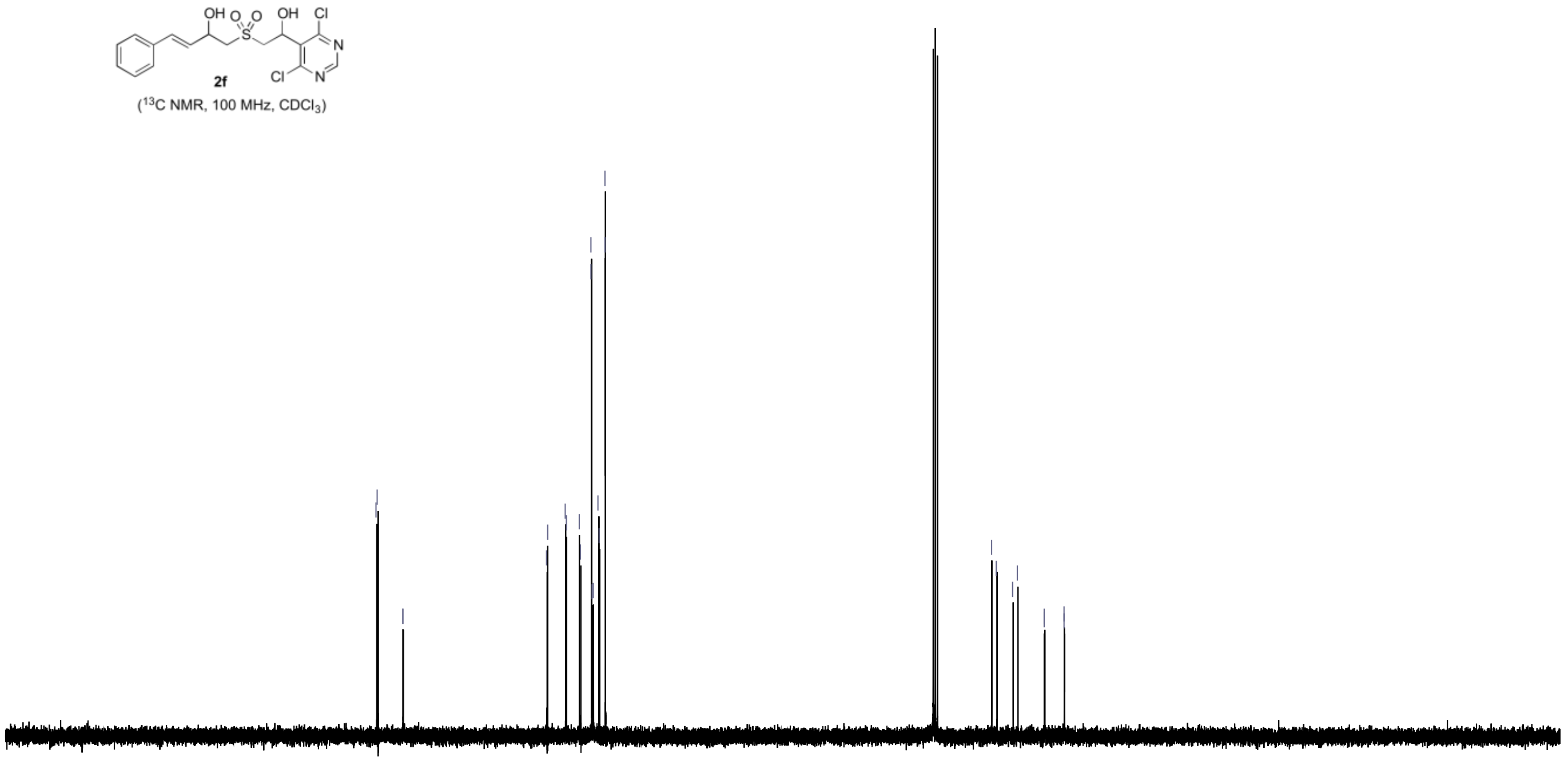


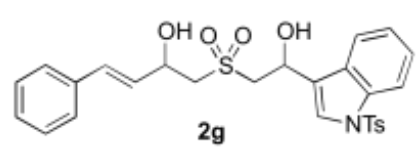

$$
\text { ('H NMR, } \left.400 \mathrm{MHz},\left(\mathrm{CD}_{3}\right)_{2} \mathrm{CO}\right)
$$
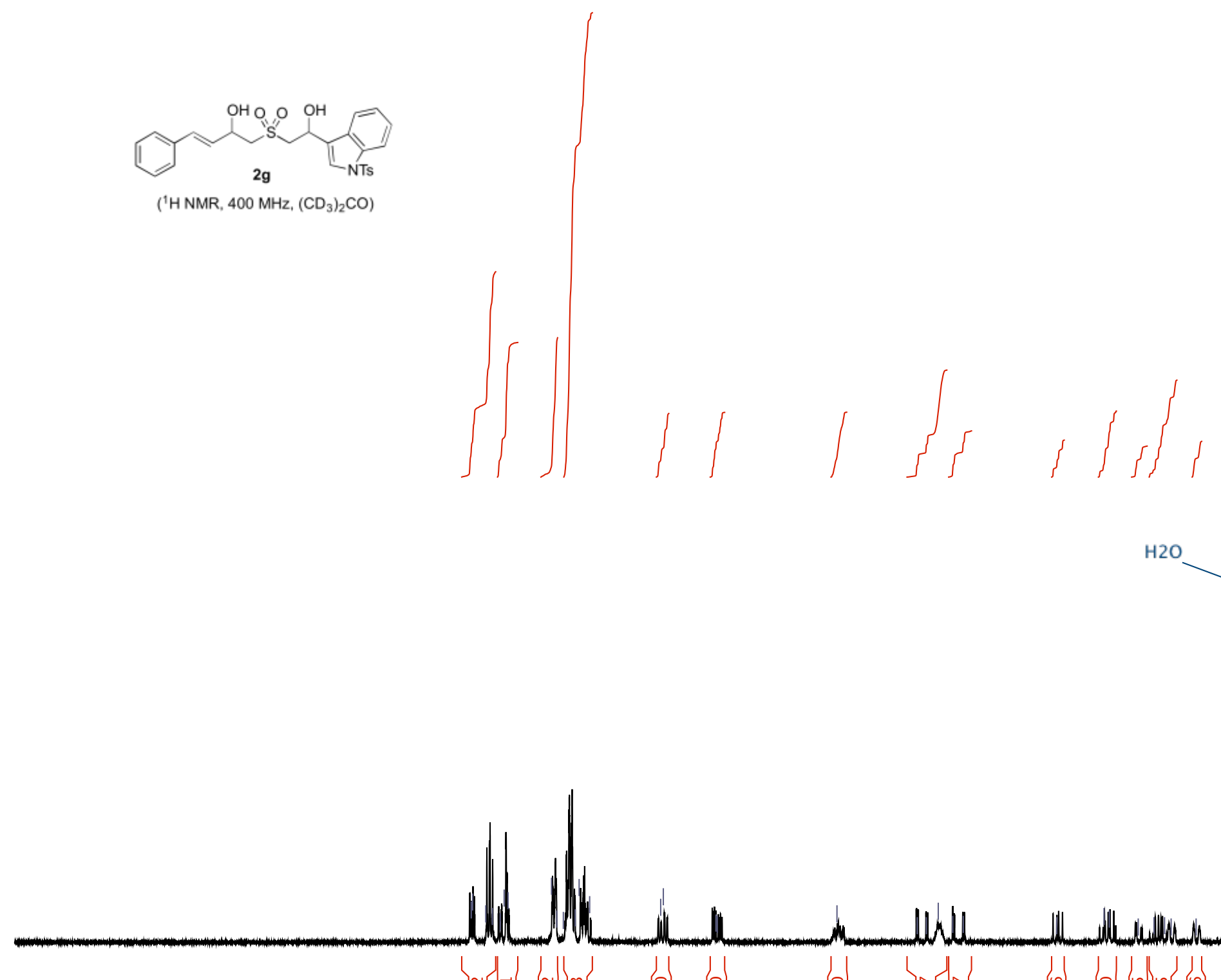

(2)

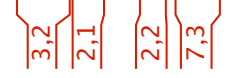




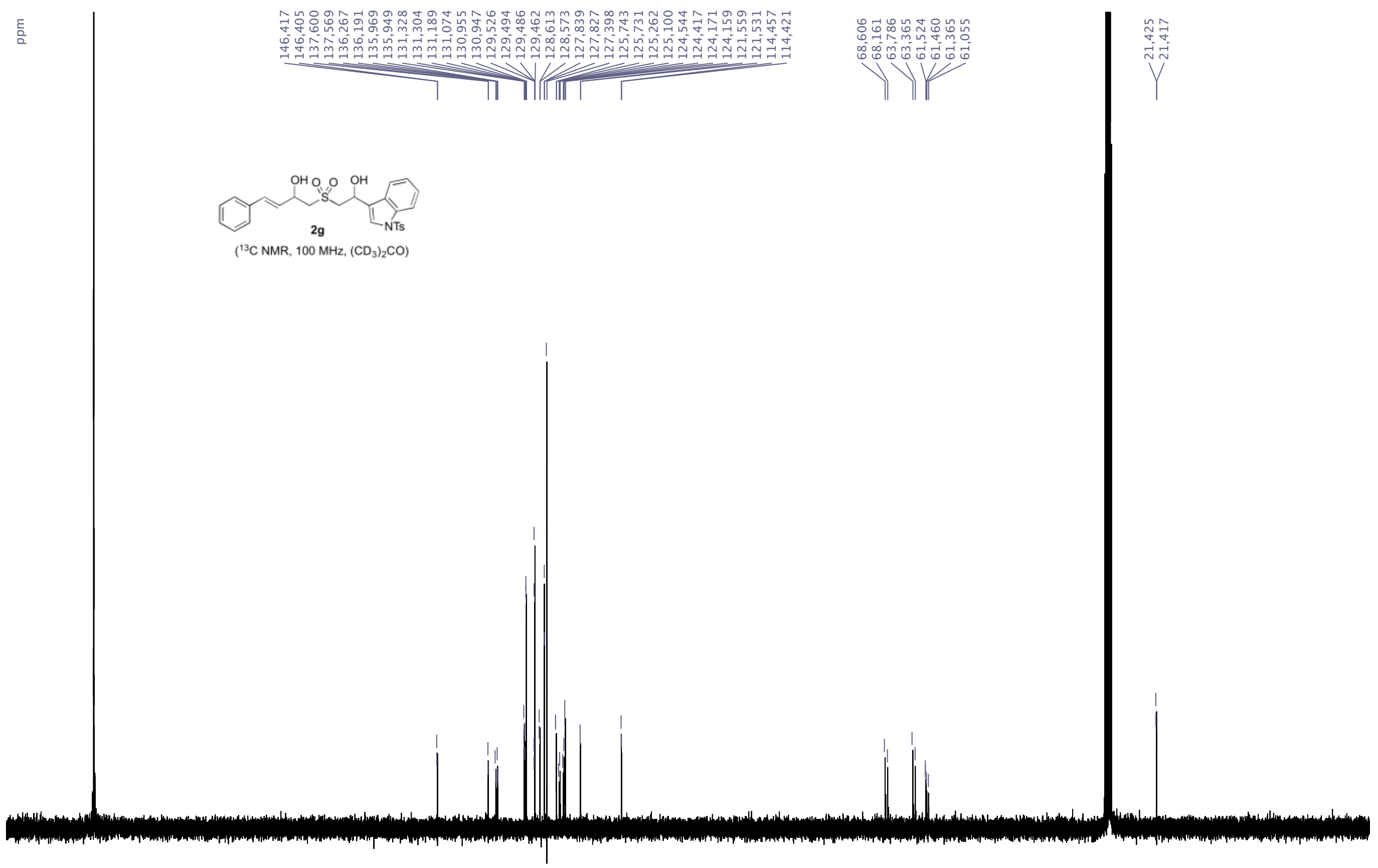




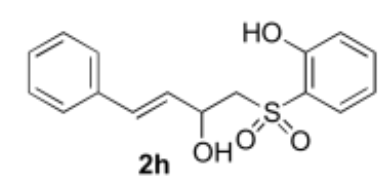

( $\left.{ }^{1} \mathrm{H} \mathrm{NMR}, 400 \mathrm{MHz},\left(\mathrm{CD}_{3}\right)_{2} \mathrm{CO}\right)$ )

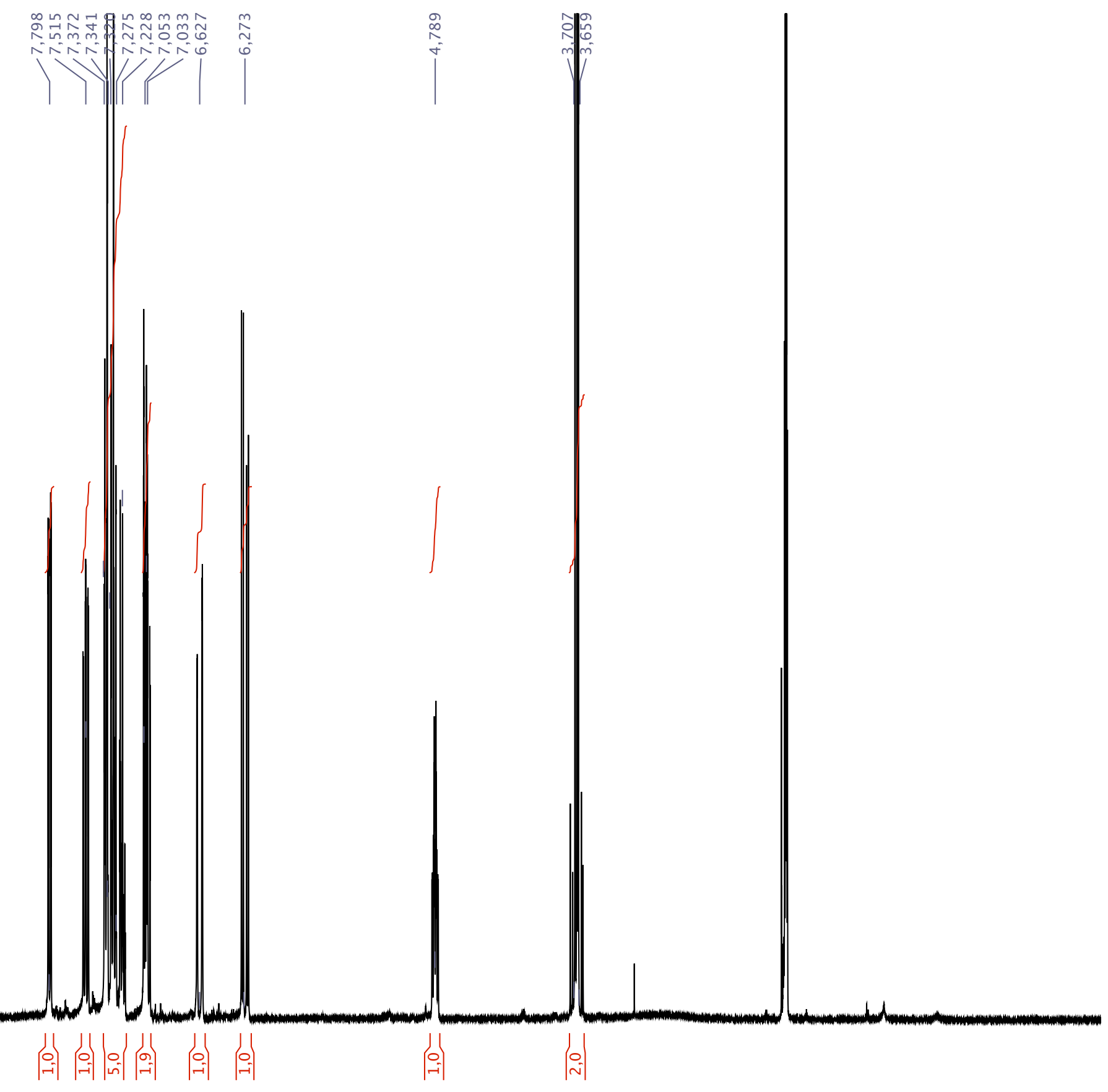



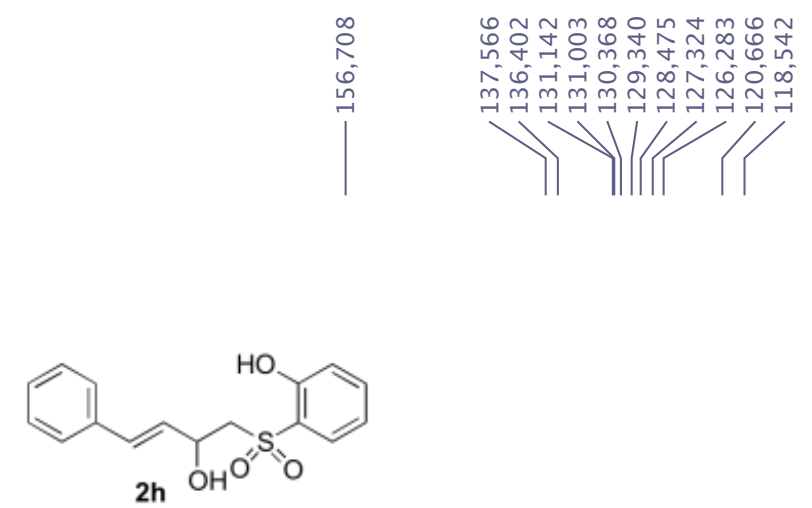

$\left({ }^{13} \mathrm{C}\right.$ NMR, $\left.\left.100 \mathrm{MHz},\left(\mathrm{CD}_{3}\right)_{2} \mathrm{CO}\right)\right)$ 
$\mathrm{OHO} \quad \mathrm{OH}$

( ${ }^{1} \mathrm{H} \mathrm{NMR}, 400 \mathrm{MHz}, \mathrm{CDCl}_{3}$ )
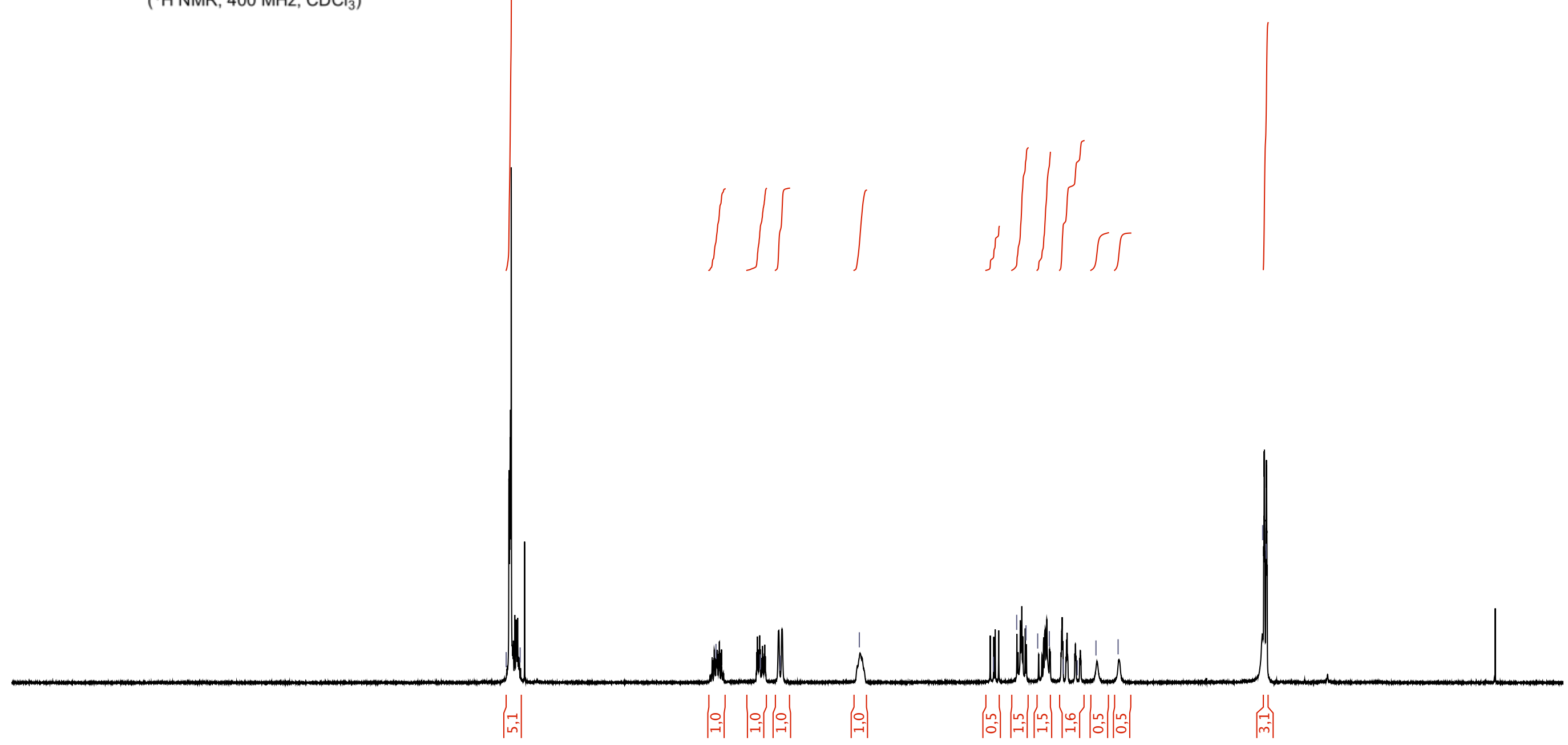


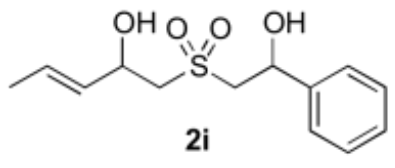

$\left({ }^{13} \mathrm{C} \mathrm{NMR}, 100 \mathrm{MHz}, \mathrm{CDCl}_{3}\right)$

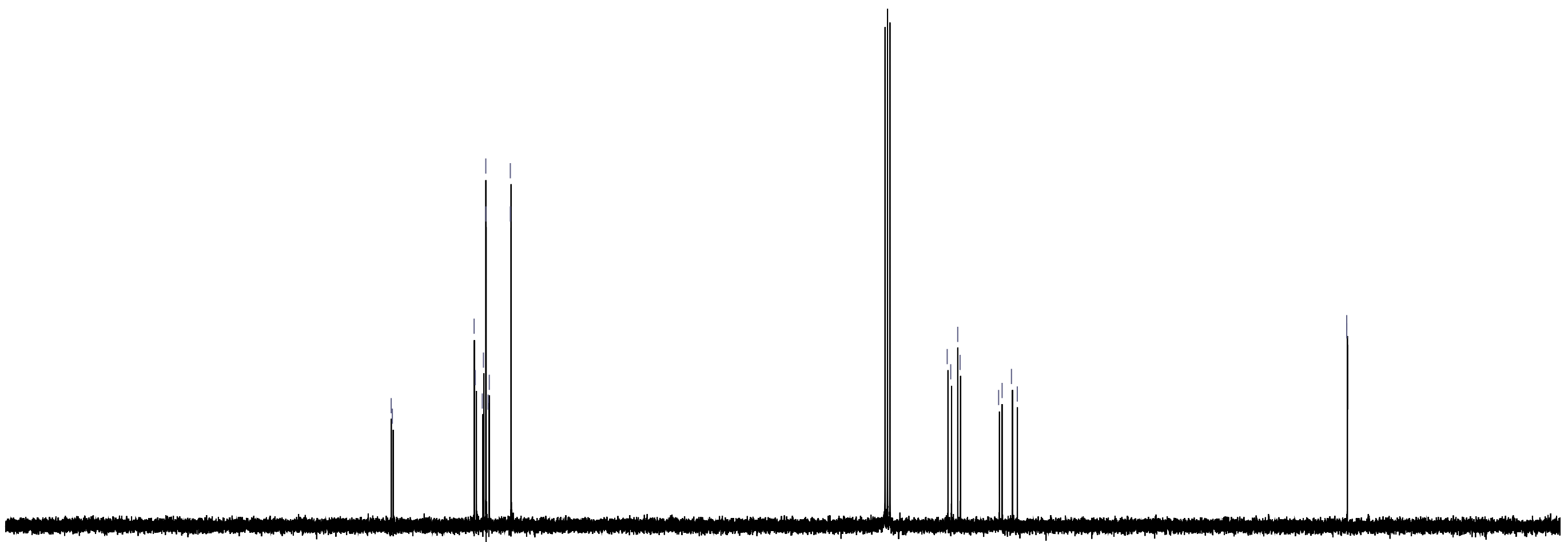




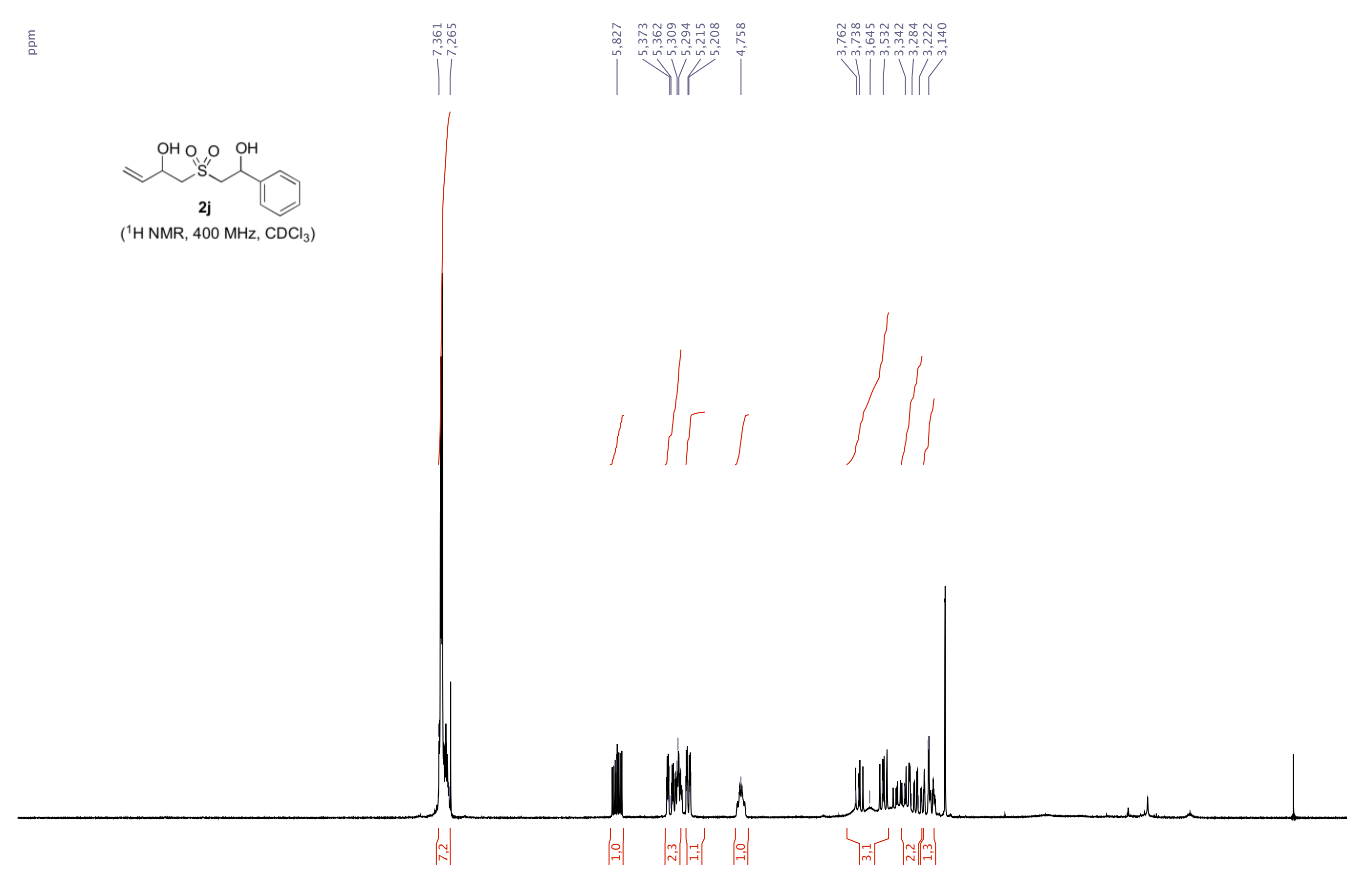





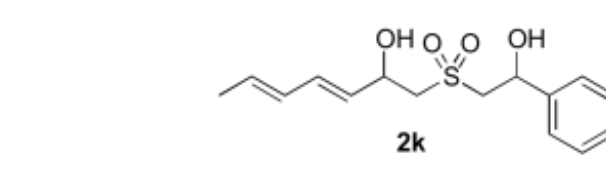

$\left({ }^{1} \mathrm{H} \mathrm{NMR}, 400 \mathrm{MHz}, \mathrm{CDCl}_{3}\right.$ )

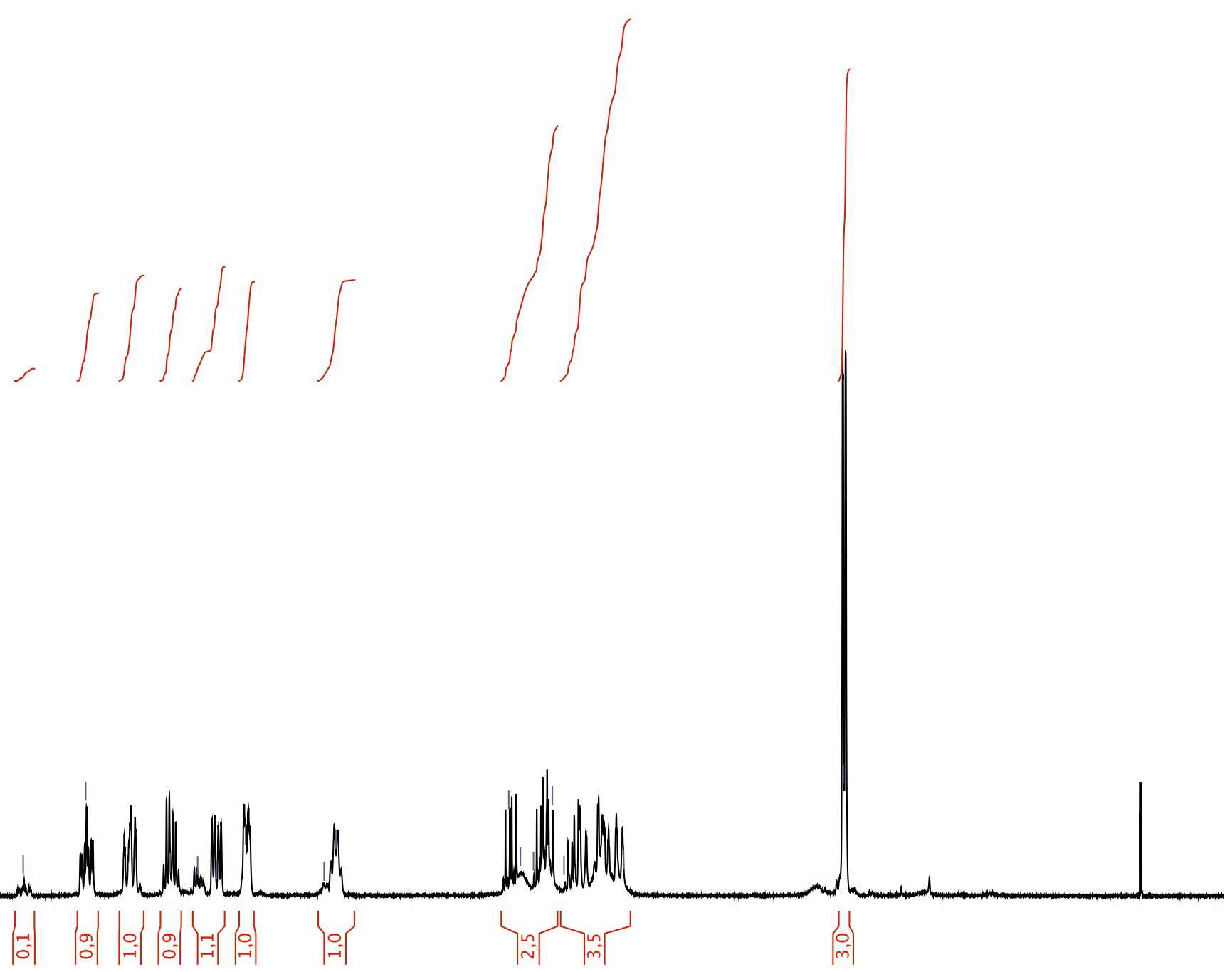




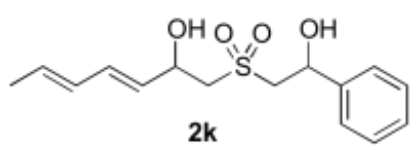

$\left({ }^{13} \mathrm{C} \mathrm{NMR}, 100 \mathrm{MHz}, \mathrm{CDCl}_{3}\right)$

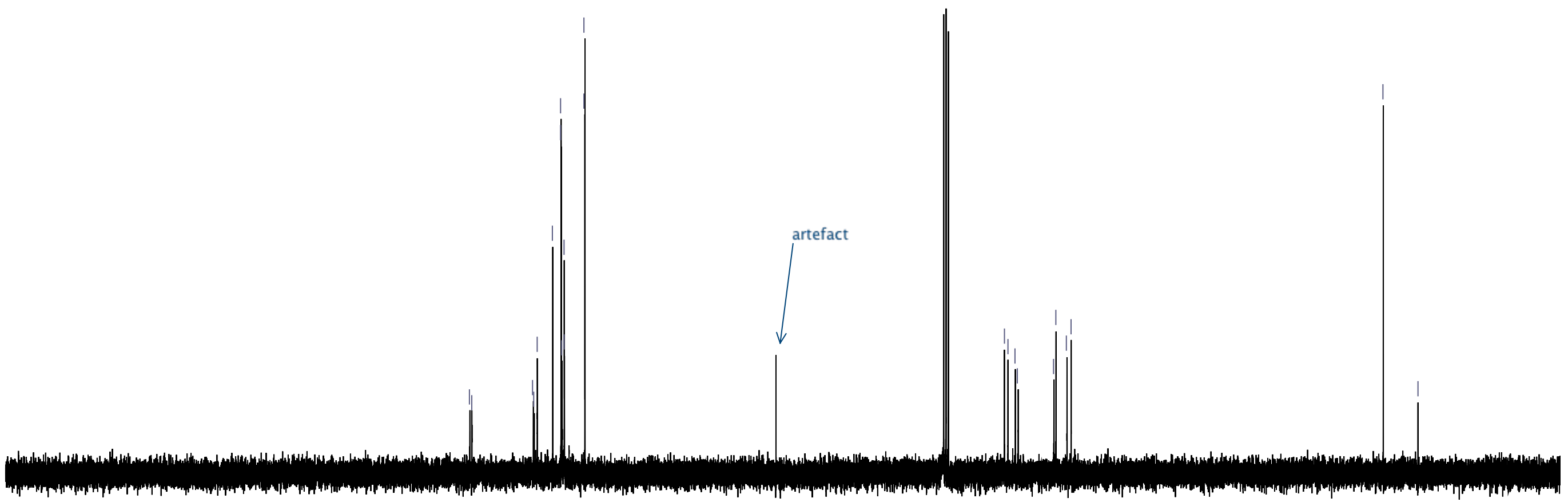




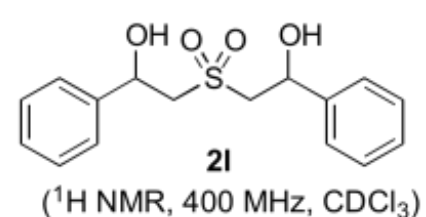

('H NMR, $400 \mathrm{MHz}, \mathrm{CDCl}_{3}$ )

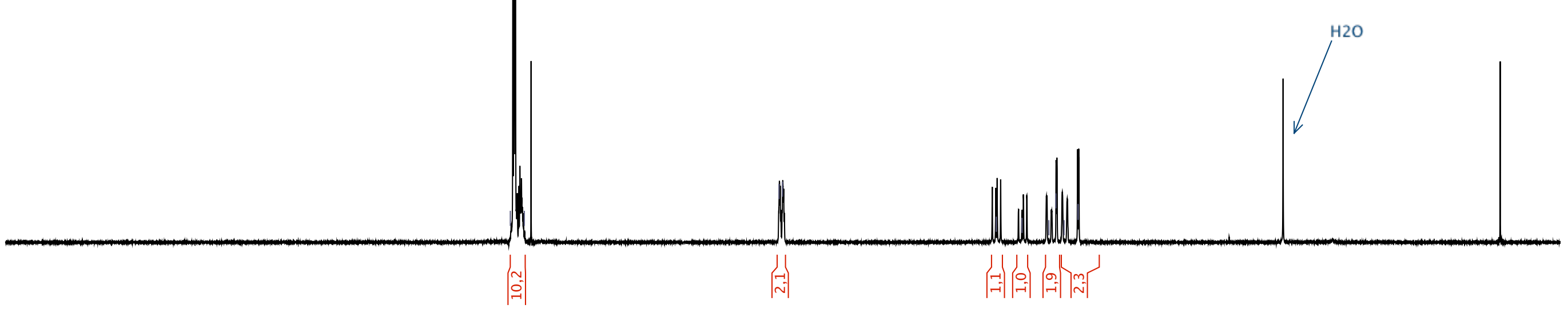


$\frac{\varepsilon}{2}$
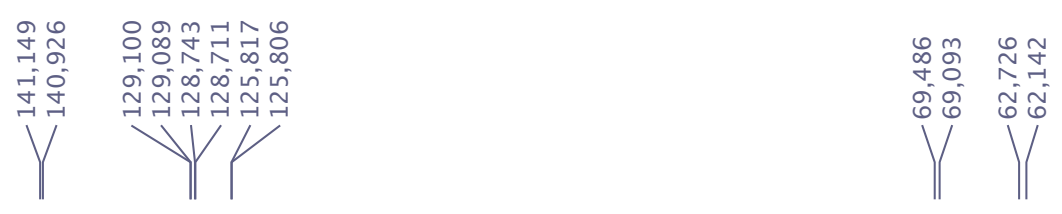<smiles>O=S(=O)(CC(O)c1ccccc1)CC(O)c1ccccc1</smiles>

$\left({ }^{13} \mathrm{C} \mathrm{NMR}, 100 \mathrm{MHz}, \mathrm{CDCl}_{3}\right)$

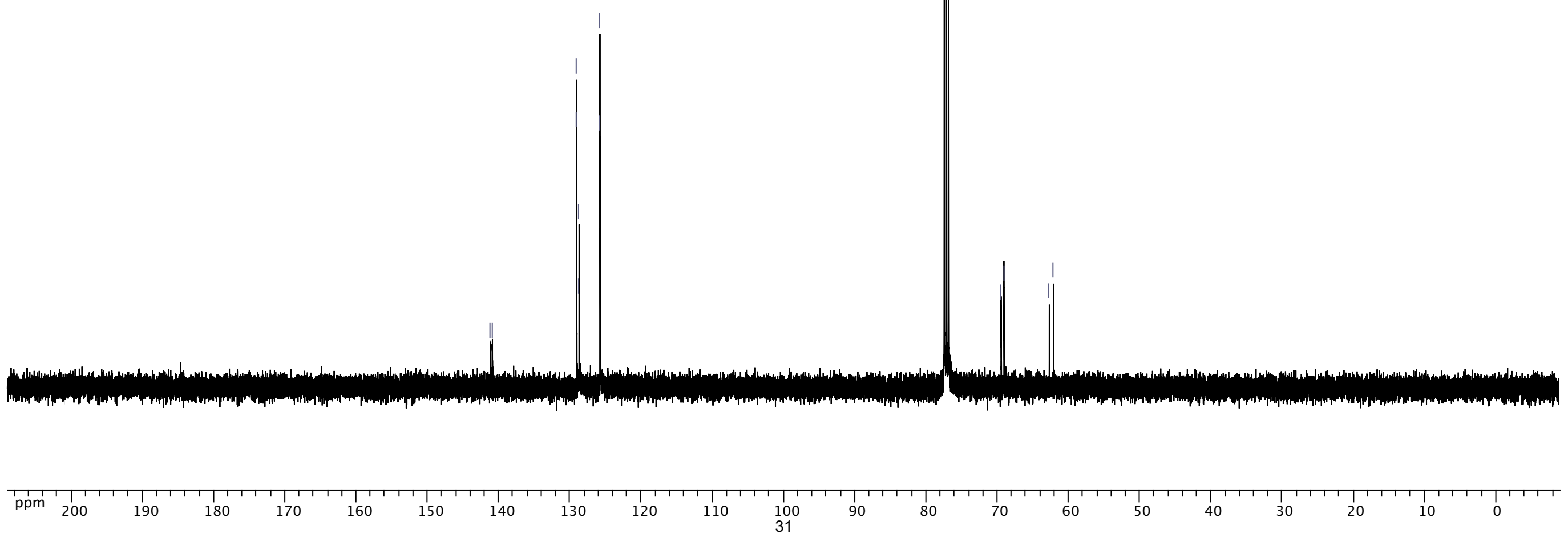




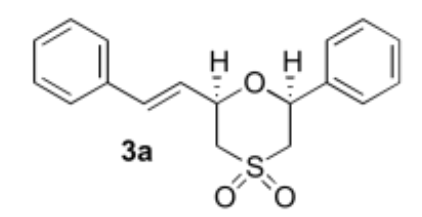

( ${ }^{1} \mathrm{H} \mathrm{NMR,}, 400 \mathrm{MHz}, \mathrm{CDCl}_{3}$ )

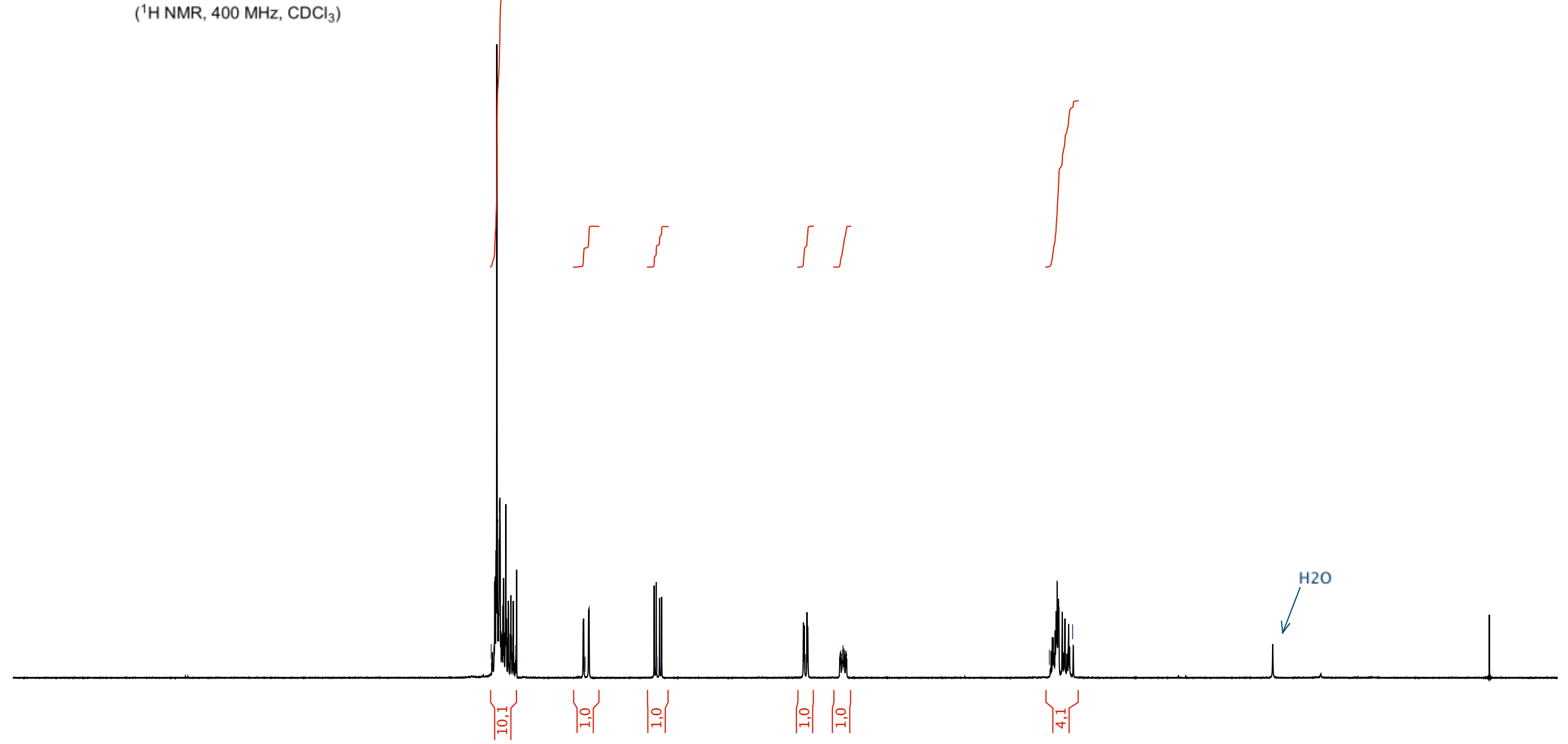




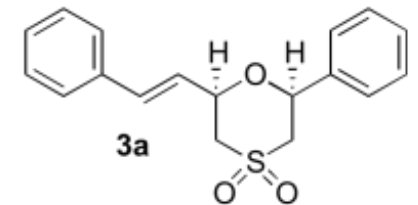

$\left({ }^{13} \mathrm{C} \mathrm{NMR}, 100 \mathrm{MHz}, \mathrm{CDCl}_{3}\right)$

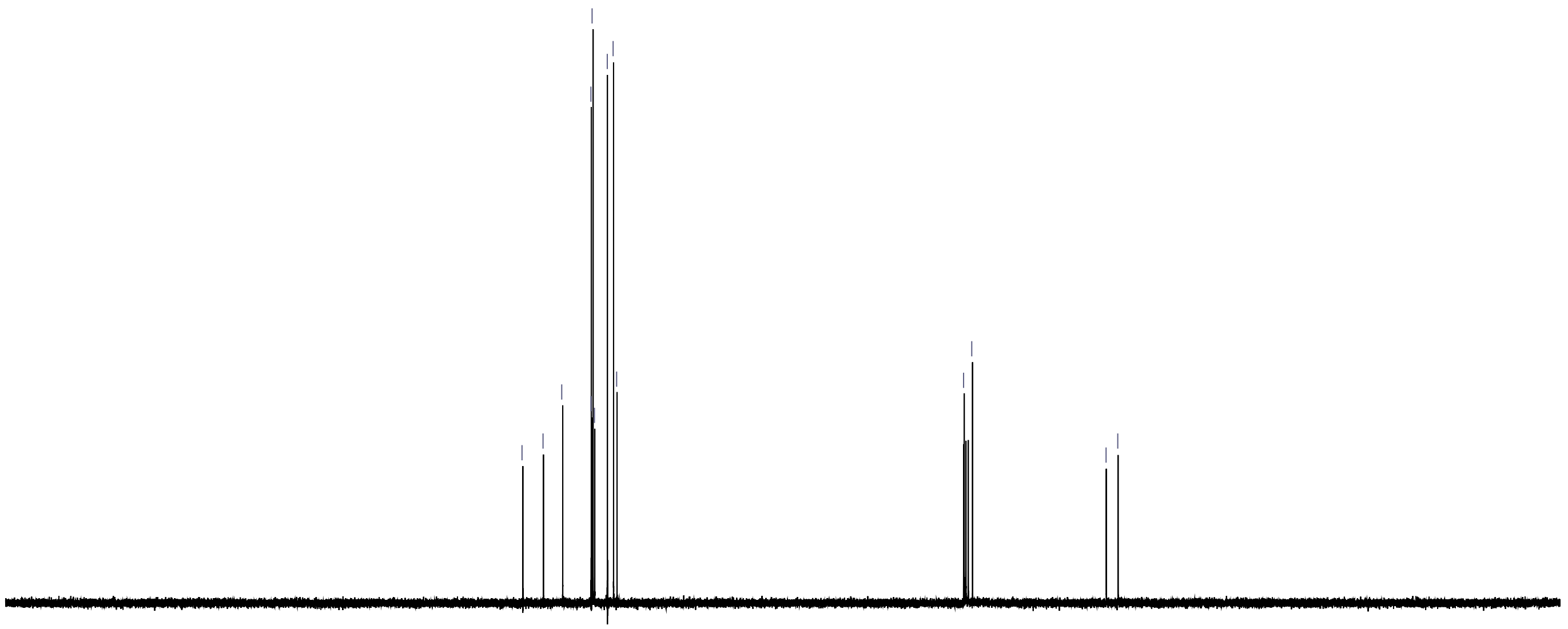




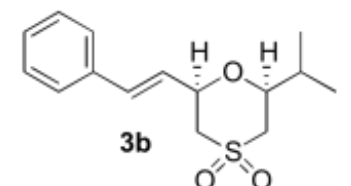

$\left({ }^{1} \mathrm{H} \mathrm{NMR}, 400 \mathrm{MHz}, \mathrm{CDCl}_{3}\right.$ )
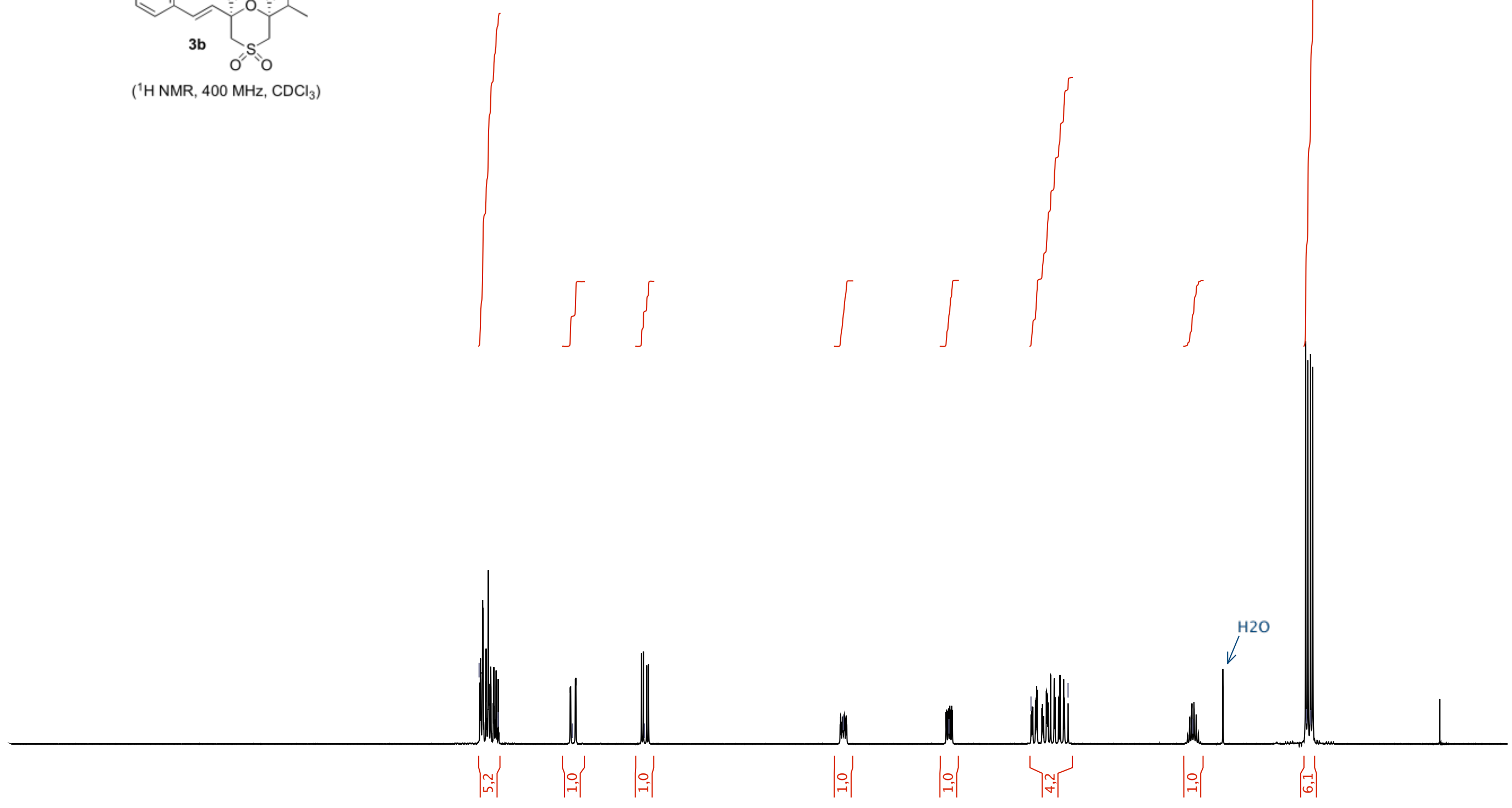


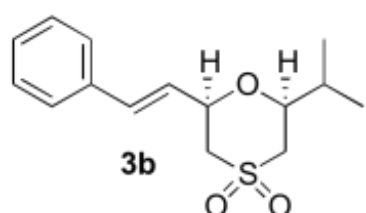

$\left({ }^{13} \mathrm{C} \mathrm{NMR}, 100 \mathrm{MHz}, \mathrm{CDCl}_{3}\right.$ )

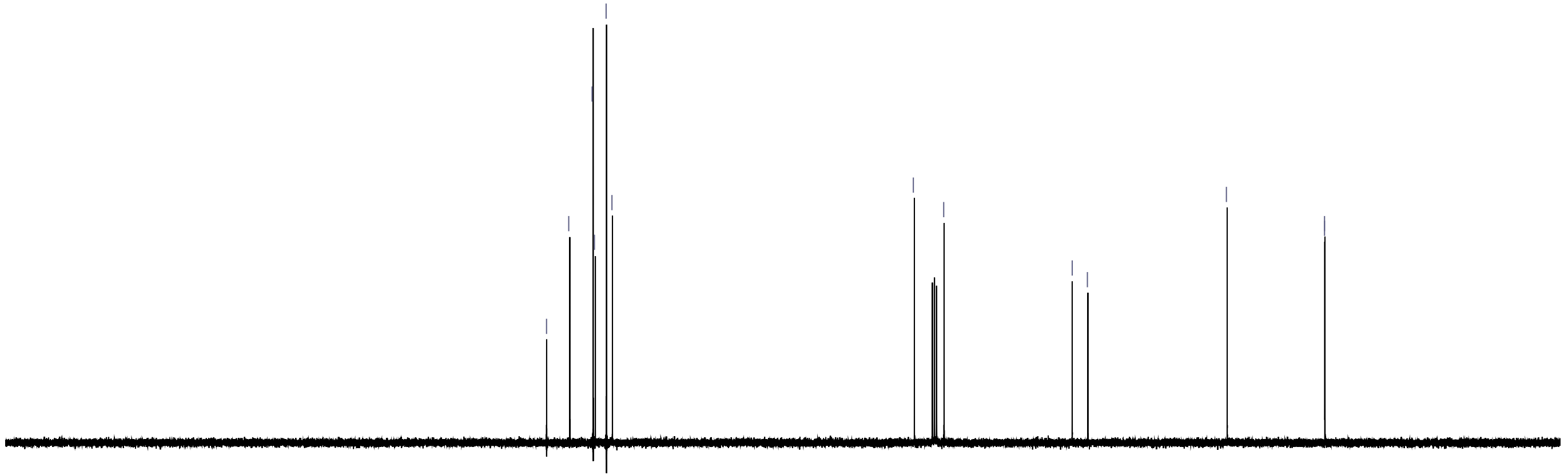




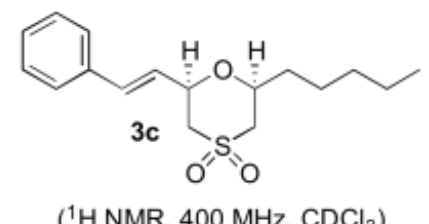

( ${ }^{1} \mathrm{H} \mathrm{NMR}, 400 \mathrm{MHz}, \mathrm{CDCl}_{3}$ )

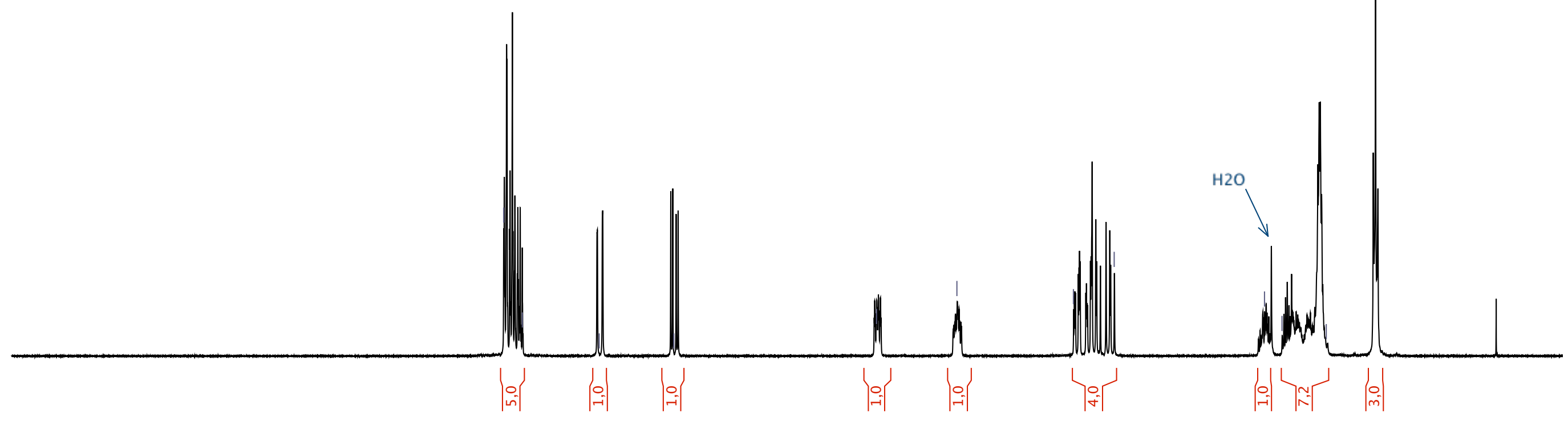




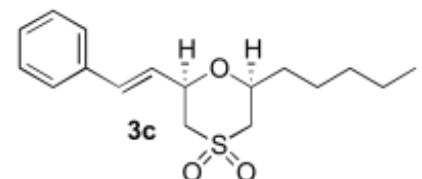

$\left({ }^{13} \mathrm{C} \mathrm{NMR}, 100 \mathrm{MHz}, \mathrm{CDCl}_{3}\right)$

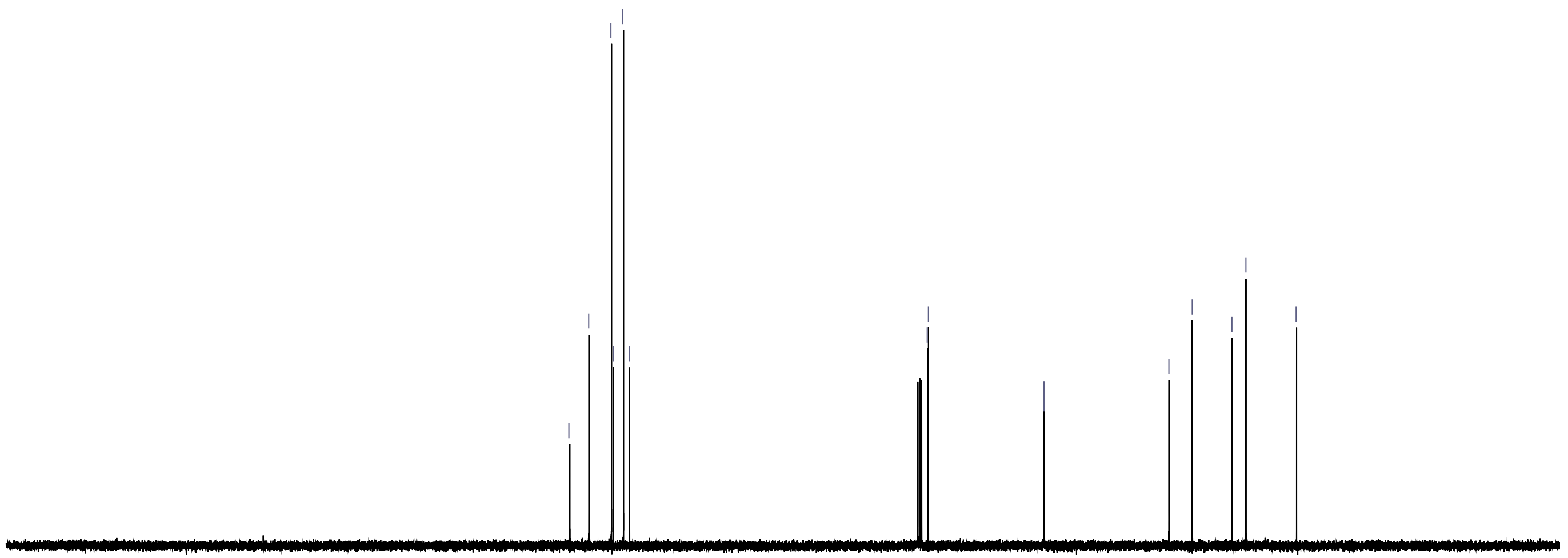




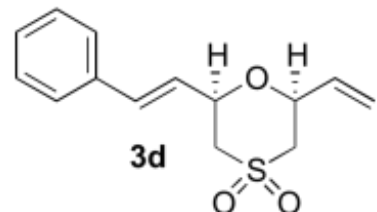

$\left({ }^{1} \mathrm{H} \mathrm{NMR}, 400 \mathrm{MHz}, \mathrm{CDCl}_{3}\right.$ )

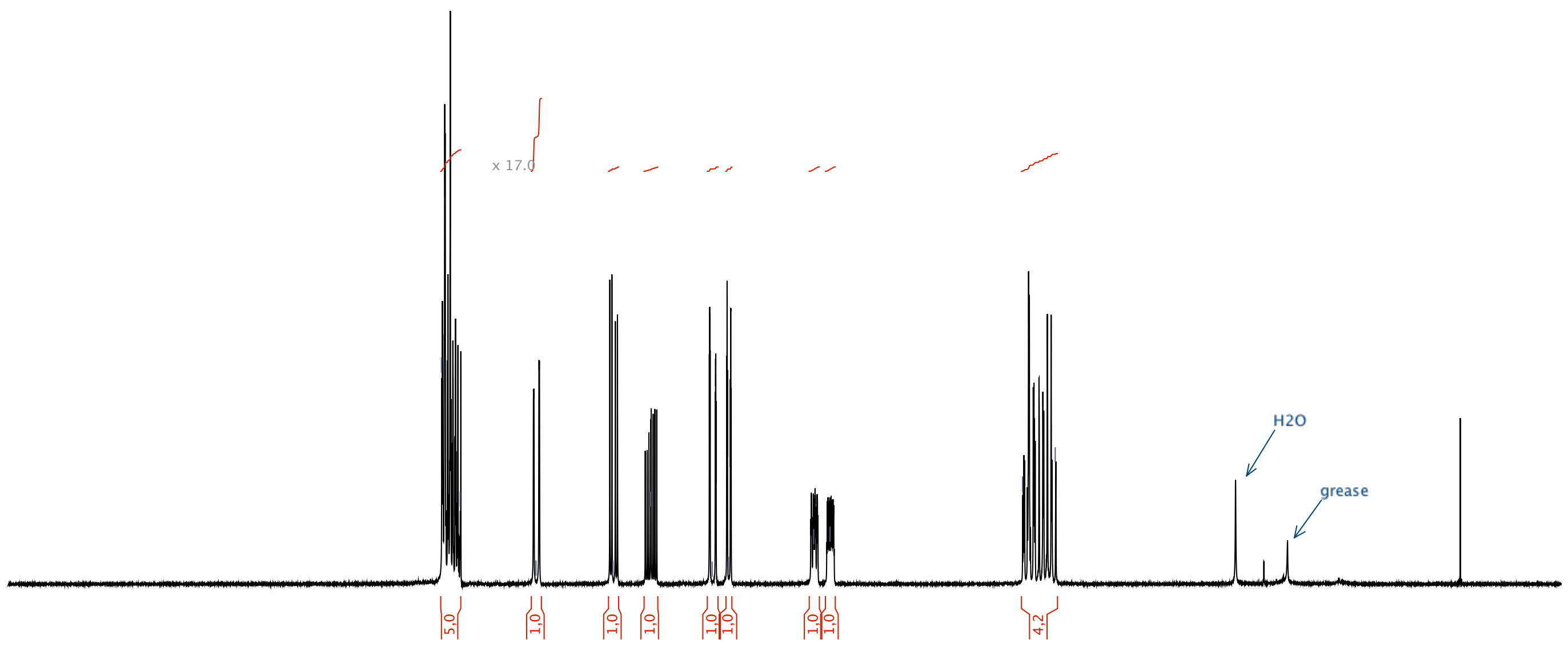




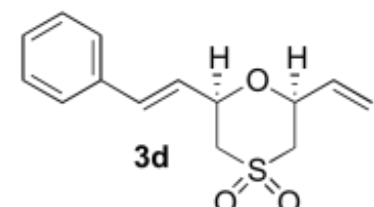

$\left({ }^{13} \mathrm{C} \mathrm{NMR}, 100 \mathrm{MHz}, \mathrm{CDCl}_{3}\right)$

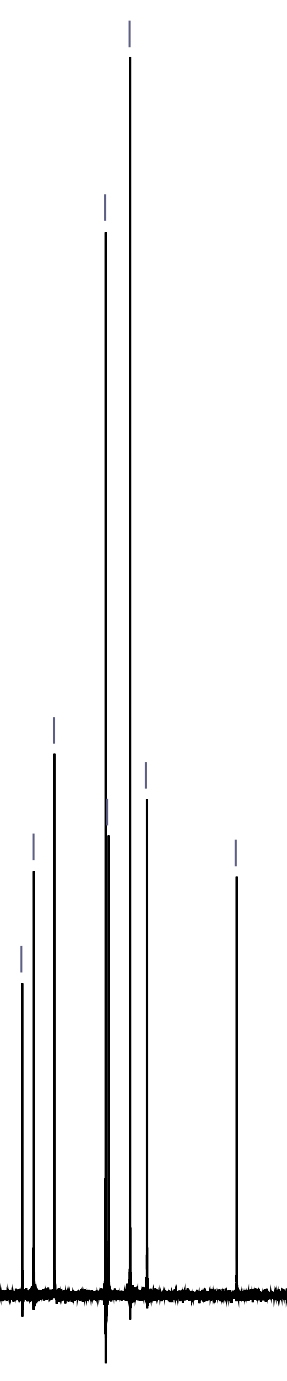




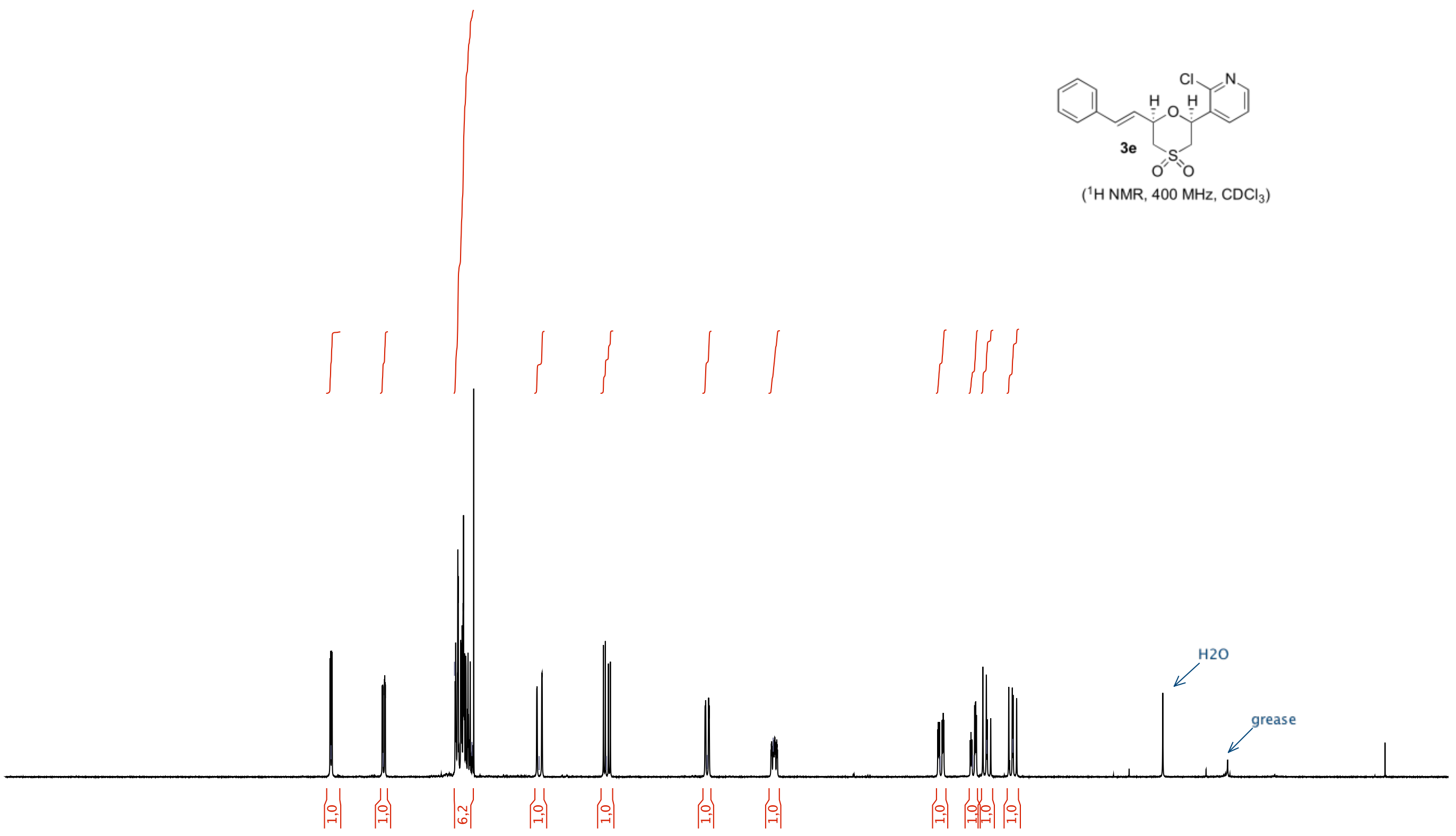



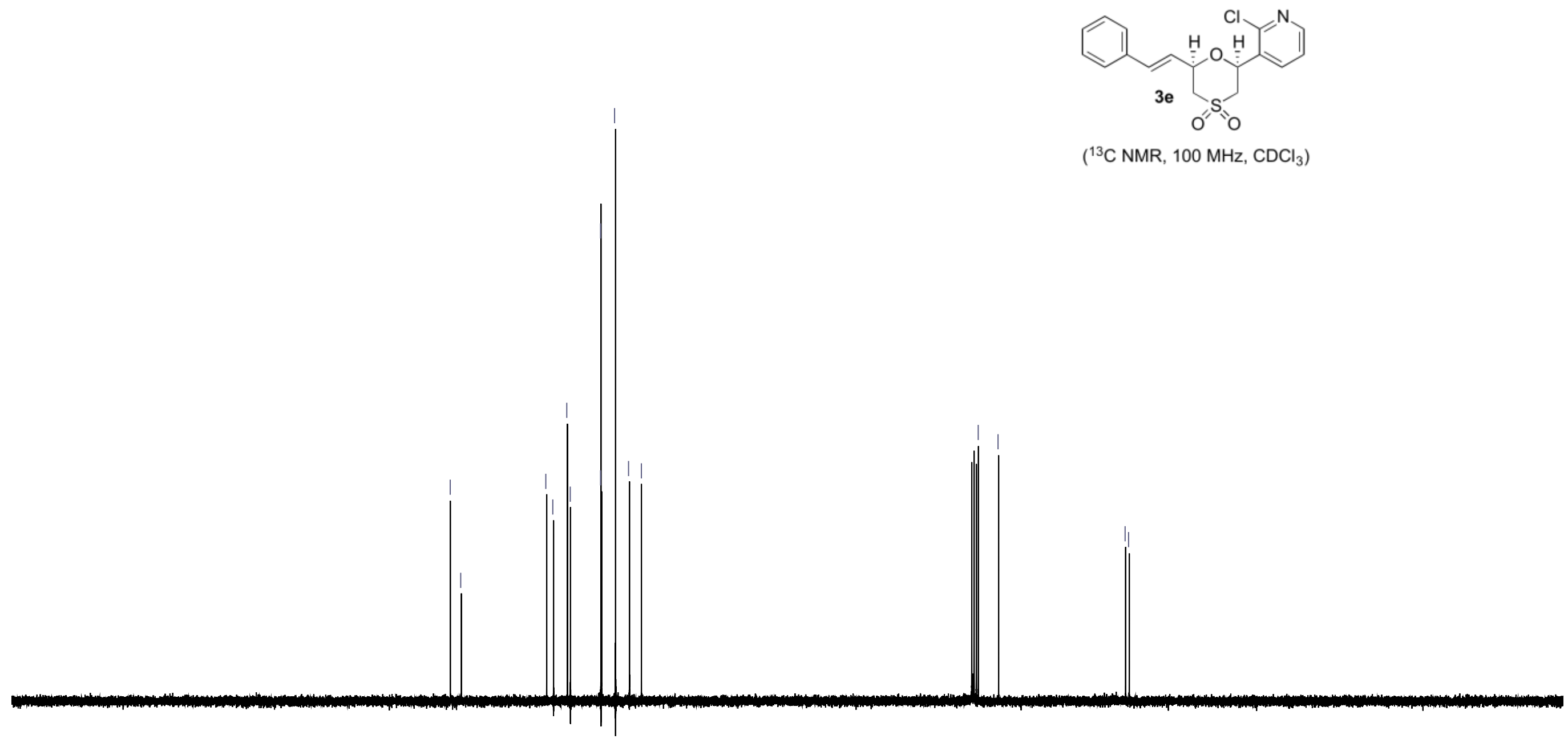

$\left({ }^{13} \mathrm{C}\right.$ NMR, $\left.100 \mathrm{MHz}, \mathrm{CDCl}_{3}\right)$ 


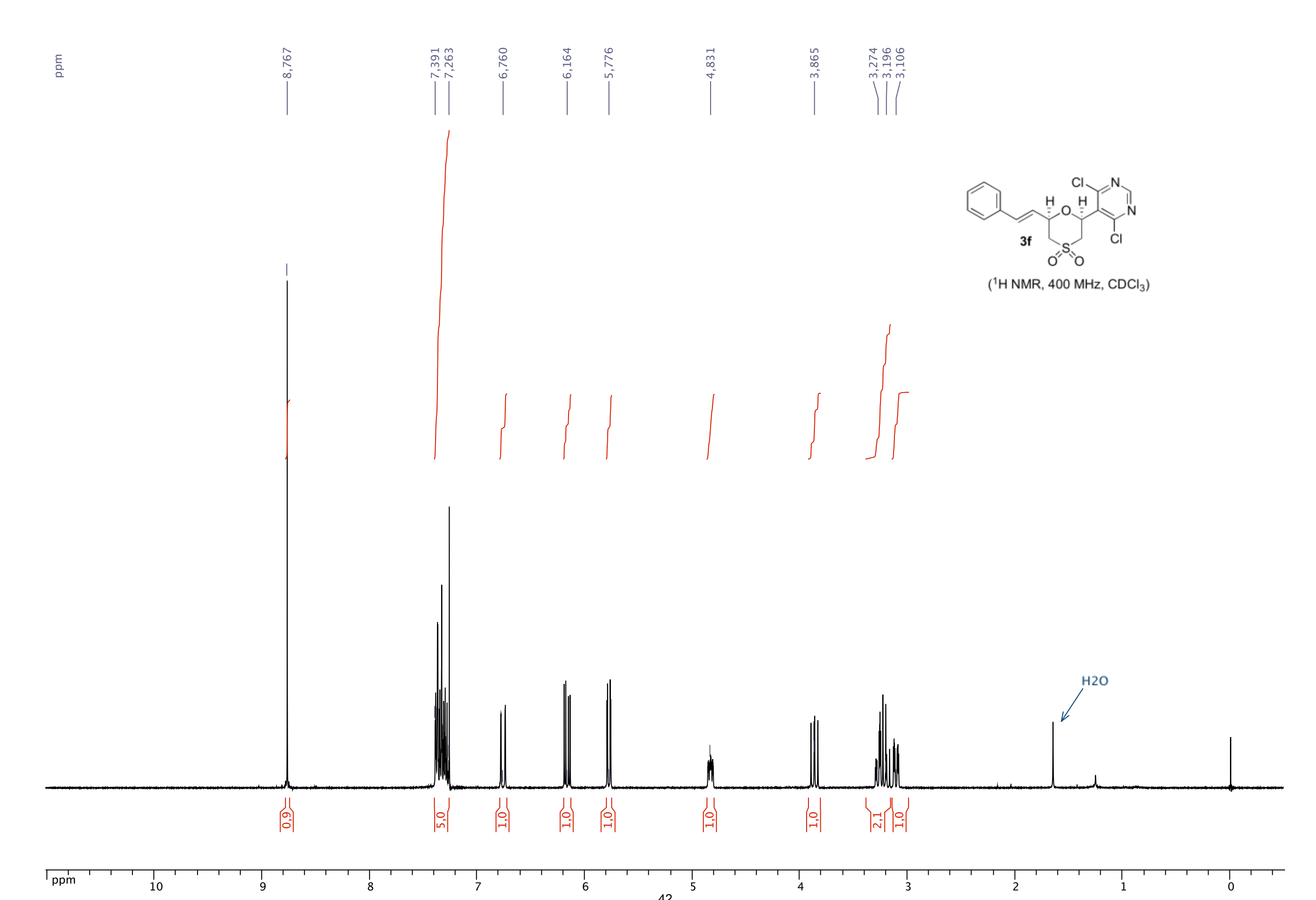




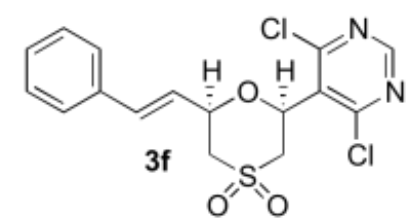

$\left({ }^{13} \mathrm{C} \mathrm{NMR}, 100 \mathrm{MHz}, \mathrm{CDCl}_{3}\right)$

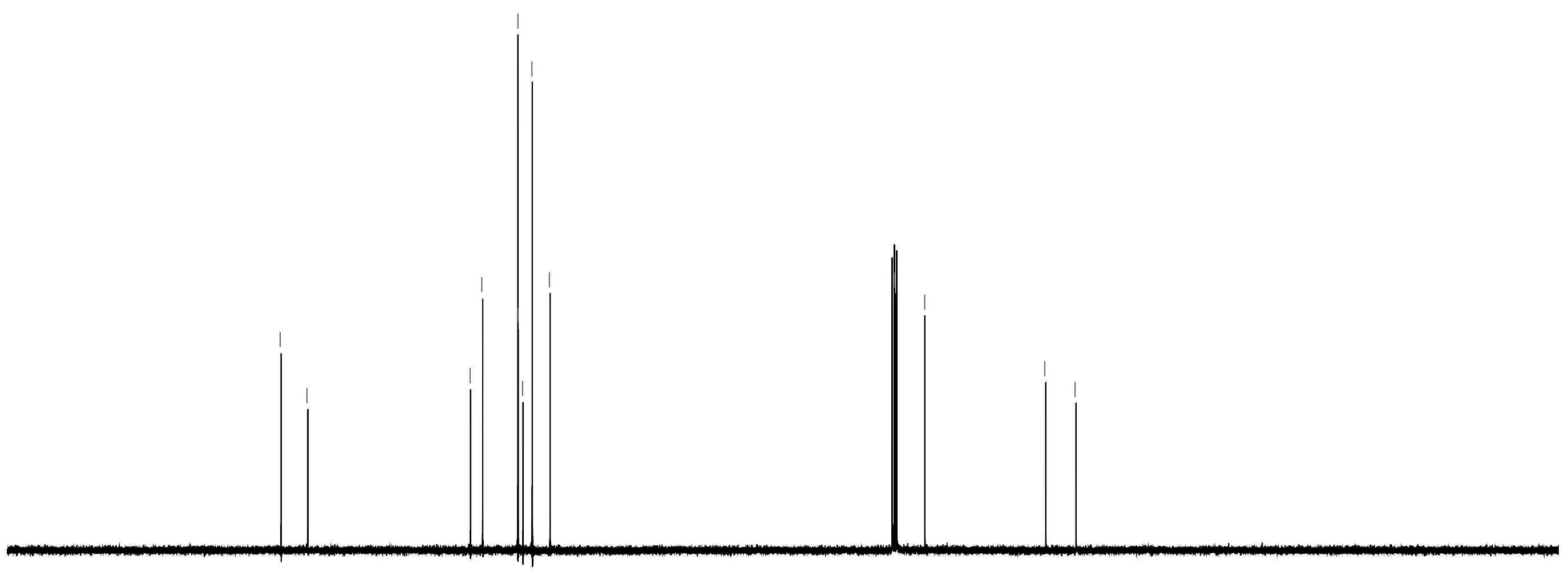




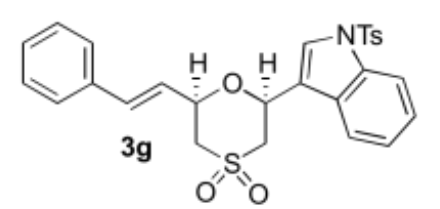

( ${ }^{1} \mathrm{H}$ NMR, $400 \mathrm{MHz}, \mathrm{CDCl}_{3}$ )
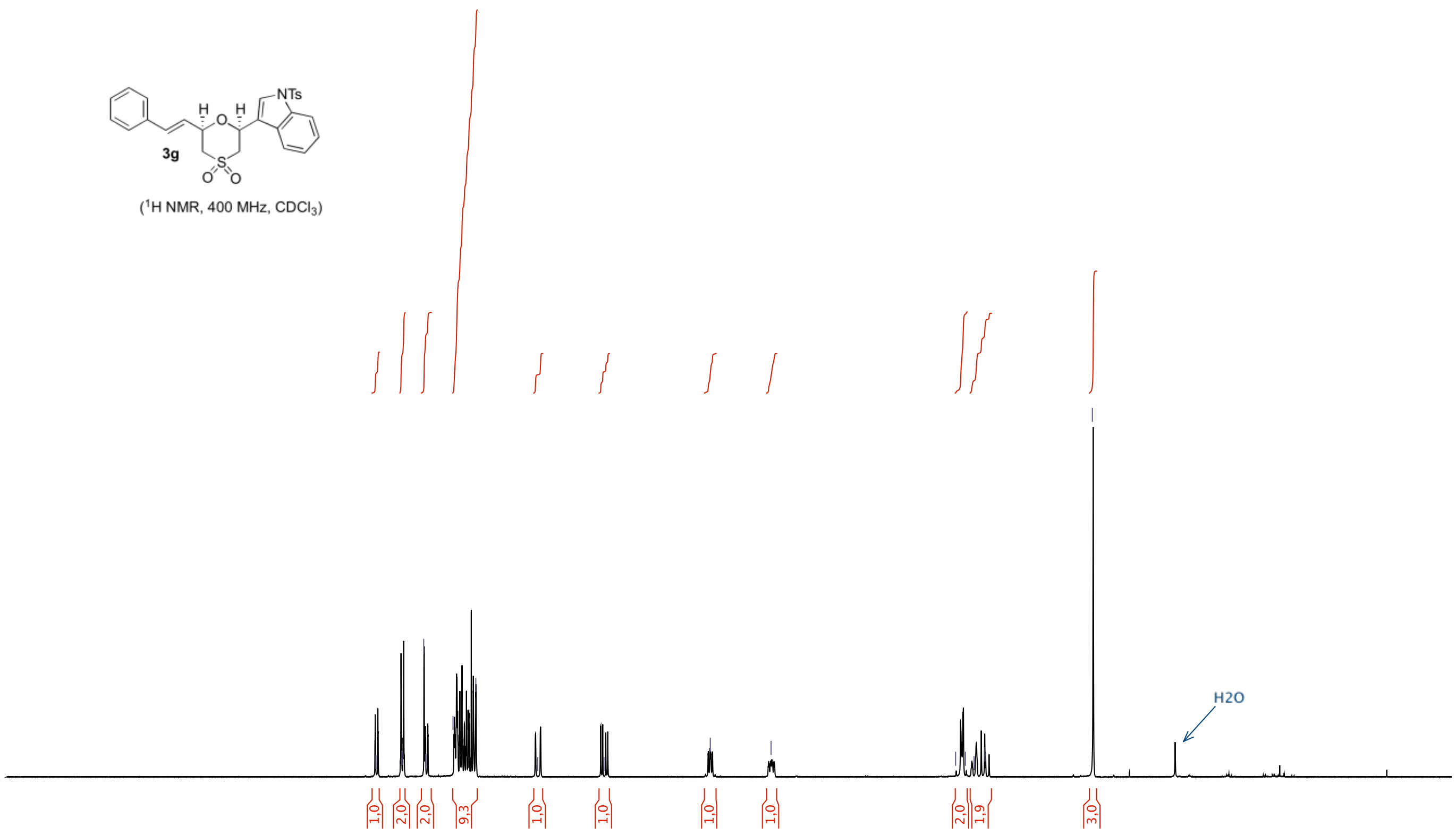


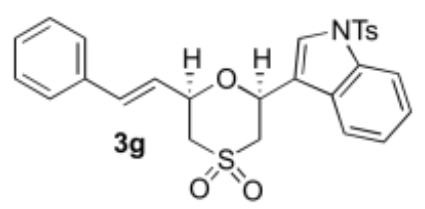

$\left({ }^{13} \mathrm{C} \mathrm{NMR,}, 100 \mathrm{MHz}, \mathrm{CDCl}_{3}\right)$

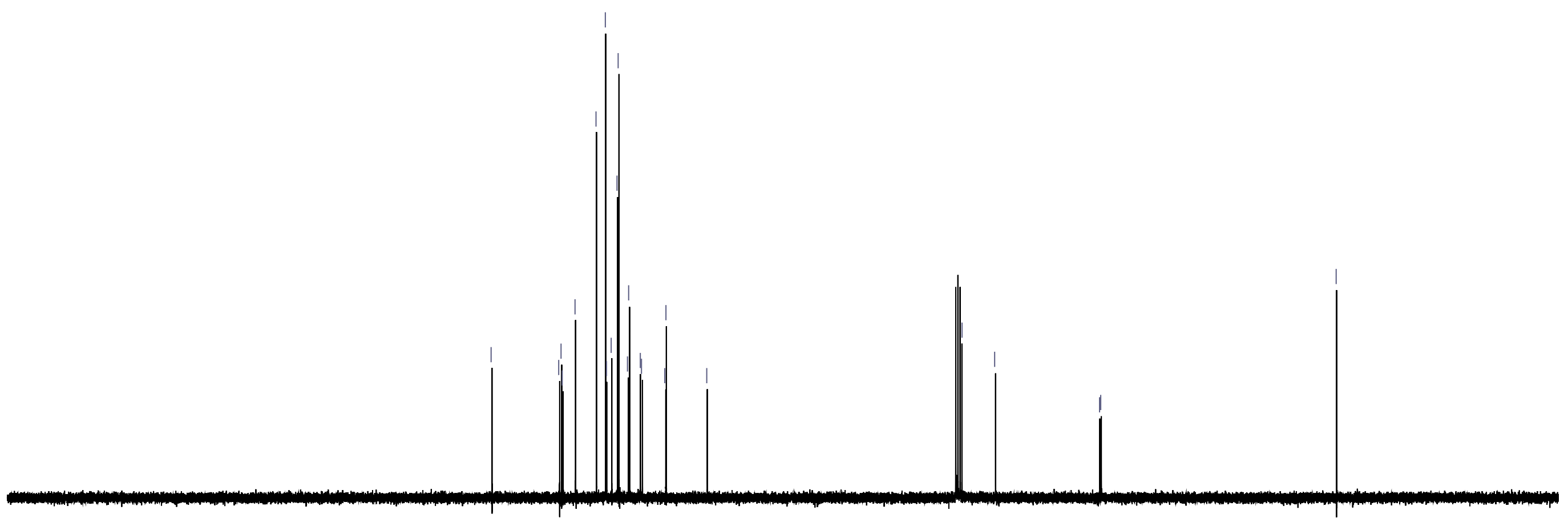




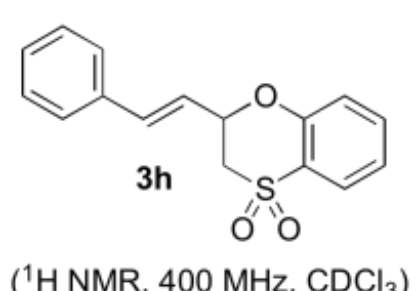

( ${ }^{1} \mathrm{H} \mathrm{NMR}, 400 \mathrm{MHz}, \mathrm{CDCl}_{3}$ )

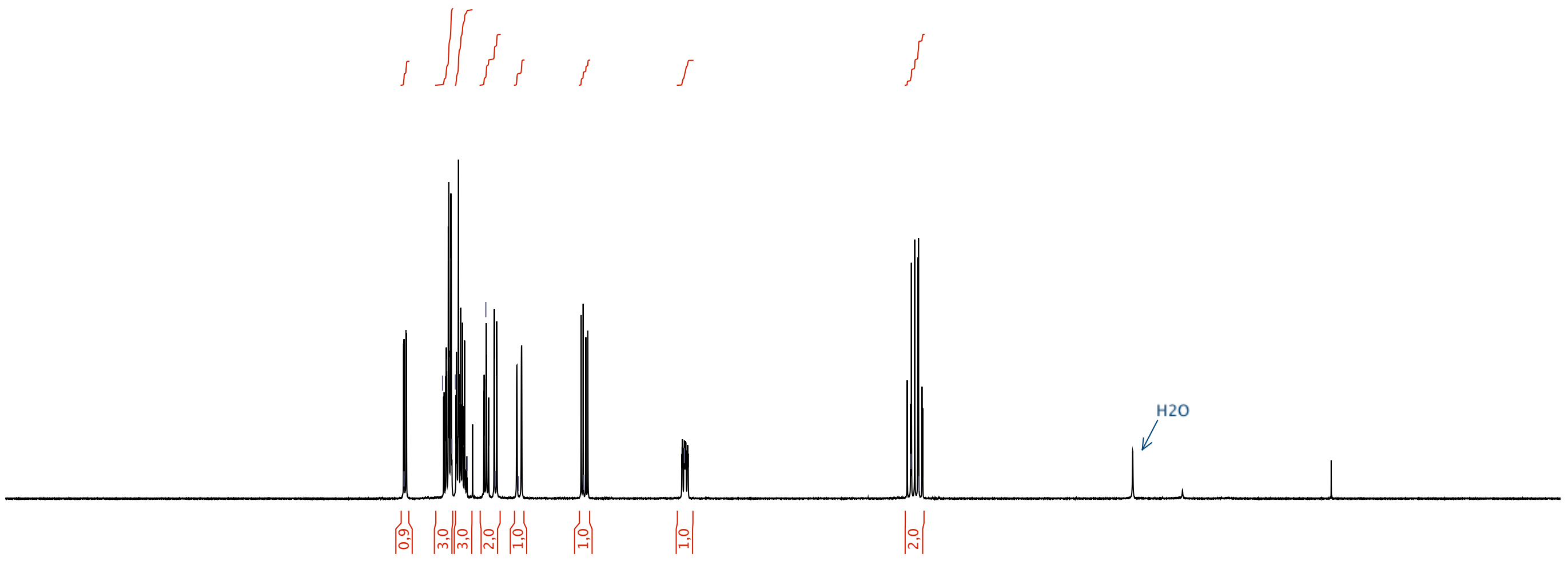




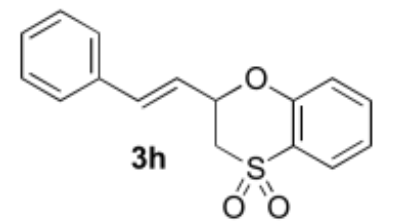

$\left({ }^{13} \mathrm{C}\right.$ NMR, $\left.100 \mathrm{MHz}, \mathrm{CDCl}_{3}\right)$

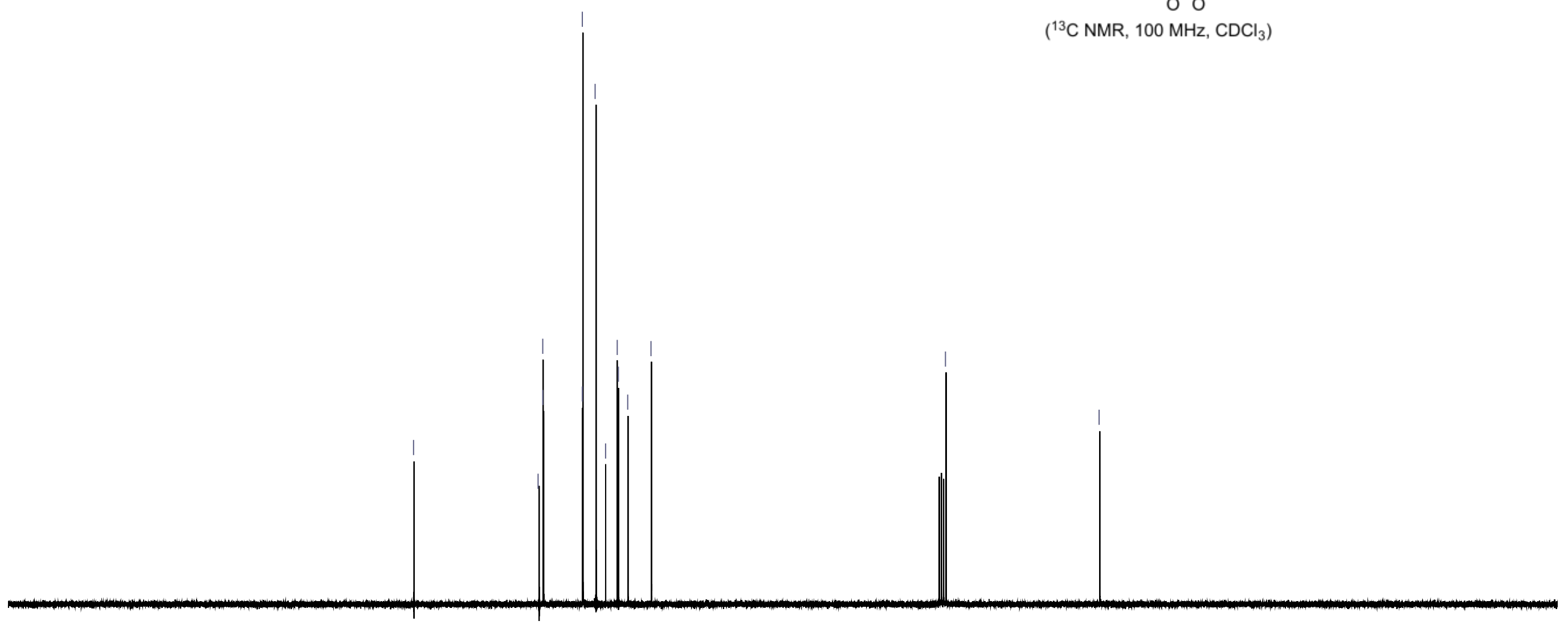




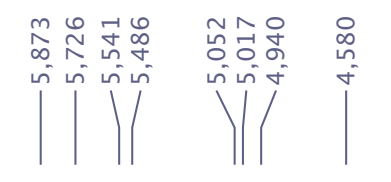

$\left.\left.\right|^{\stackrel{N}{N}}\right|^{+}$

$\left.\right|_{\substack{n \\-1}} ^{n-1}$

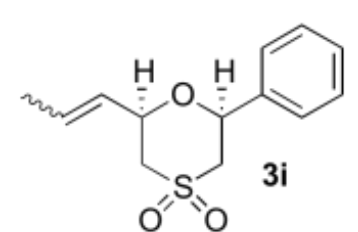

( ${ }^{1} \mathrm{H} \mathrm{NMR}, 400 \mathrm{MHz}, \mathrm{CDCl}_{3}$ )

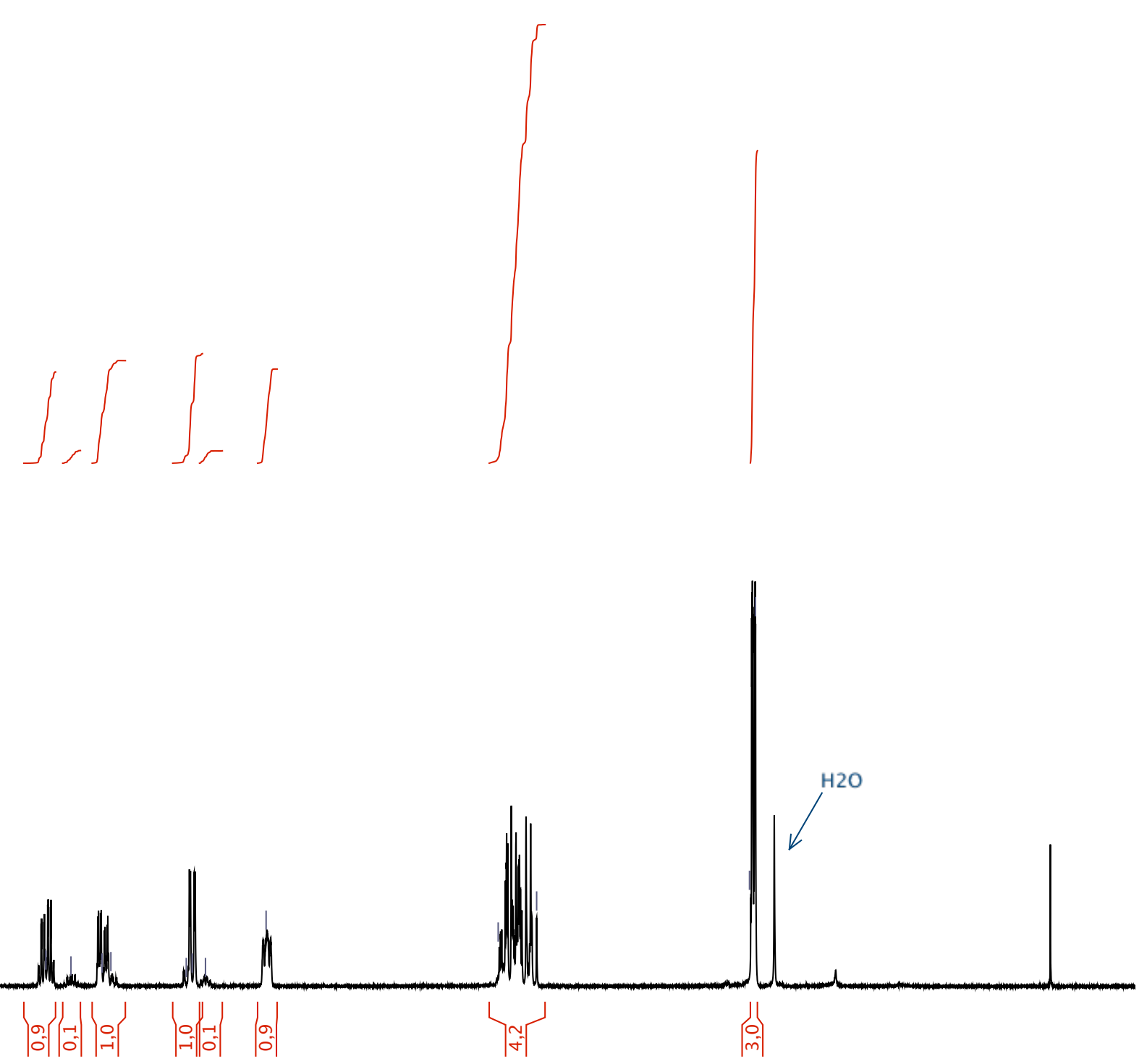




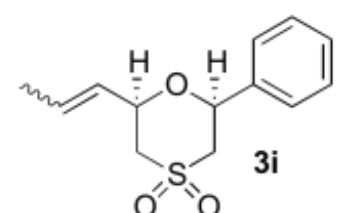

$\left({ }^{13} \mathrm{C} \mathrm{NMR}, 100 \mathrm{MHz}, \mathrm{CDCl}_{3}\right)$

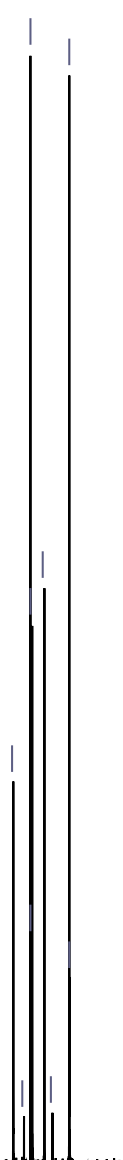




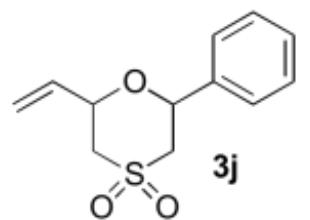

( ${ }^{1} \mathrm{H} \mathrm{NMR}, 400 \mathrm{MHz}, \mathrm{CDCl}_{3}$ )
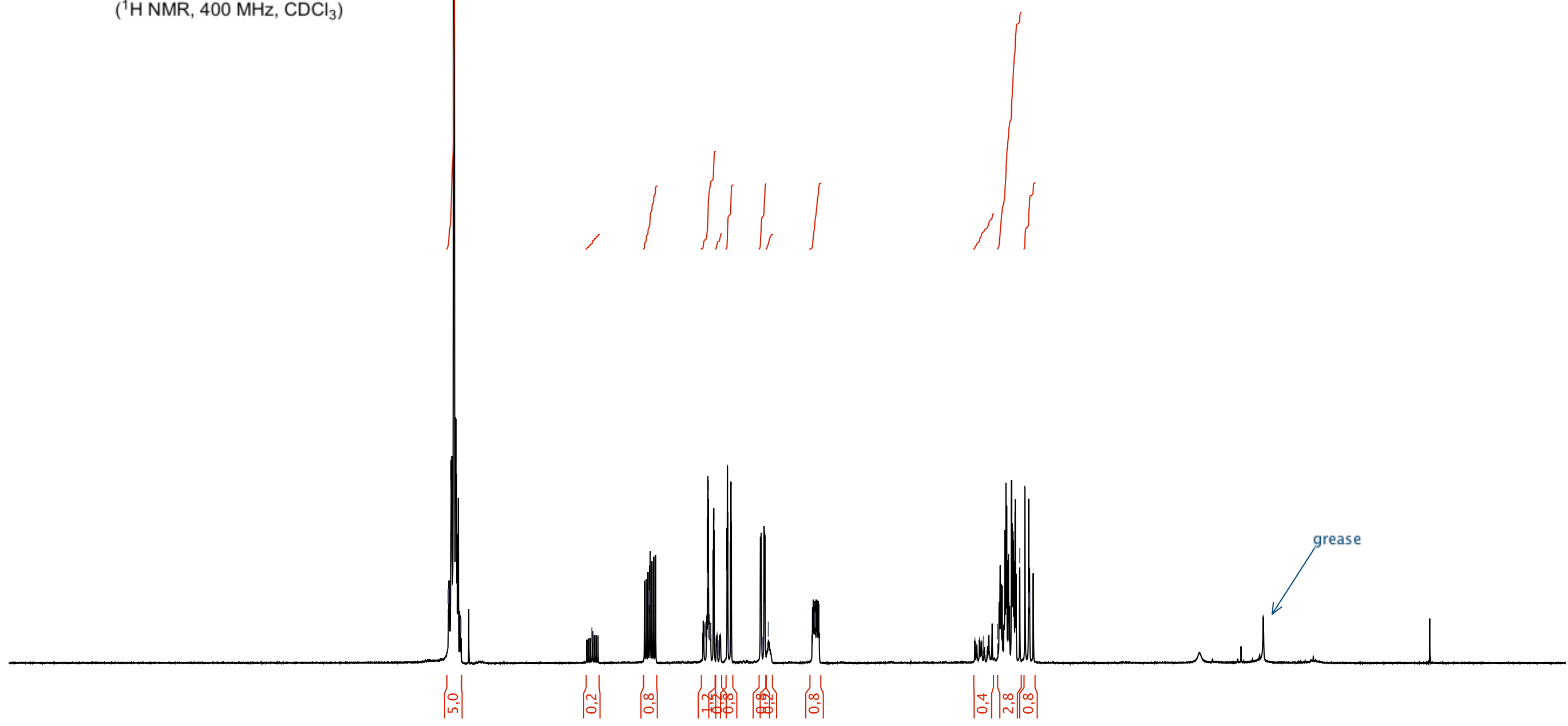


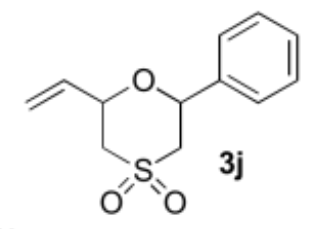

$\left({ }^{13} \mathrm{C} \mathrm{NMR}, 100 \mathrm{MHz}, \mathrm{CDCl}_{3}\right)$

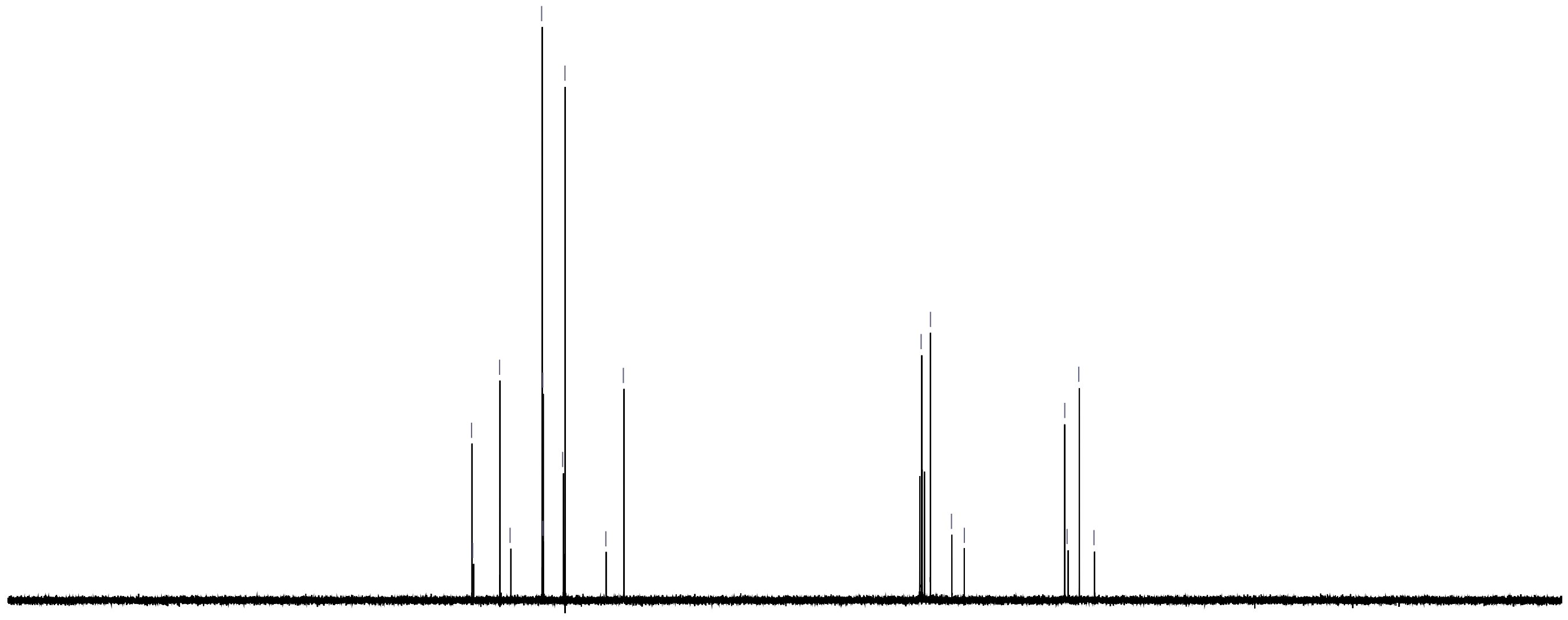




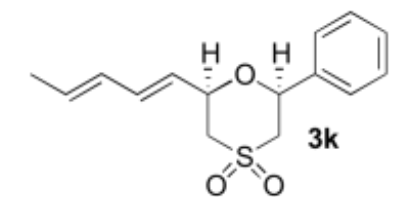

( ${ }^{1} \mathrm{H} \mathrm{NMR}, 400 \mathrm{MHz}, \mathrm{CDCl}_{3}$ )
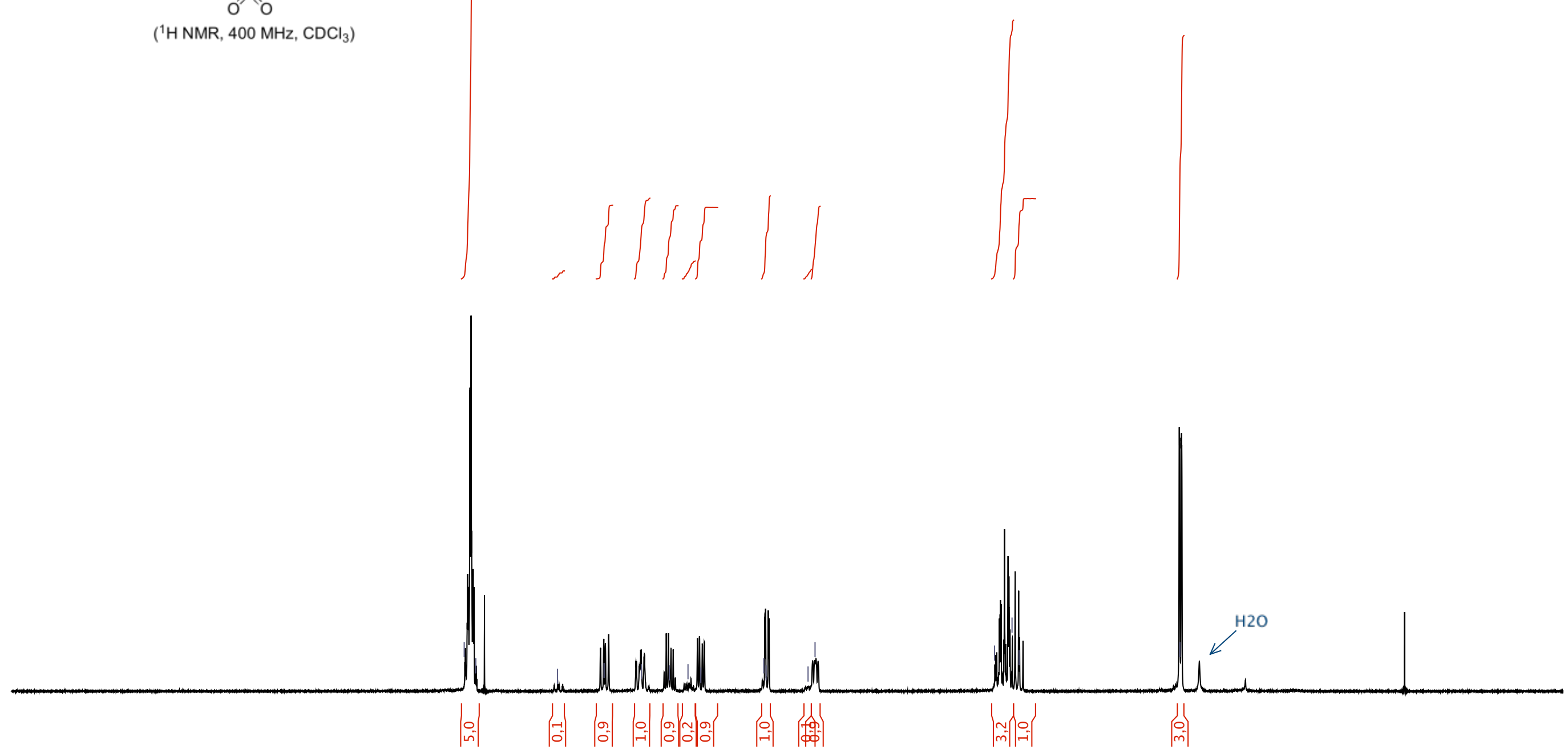


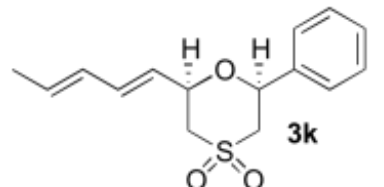

$\left({ }^{13} \mathrm{C} \mathrm{NMR}, 100 \mathrm{MHz}, \mathrm{CDCl}_{3}\right)$

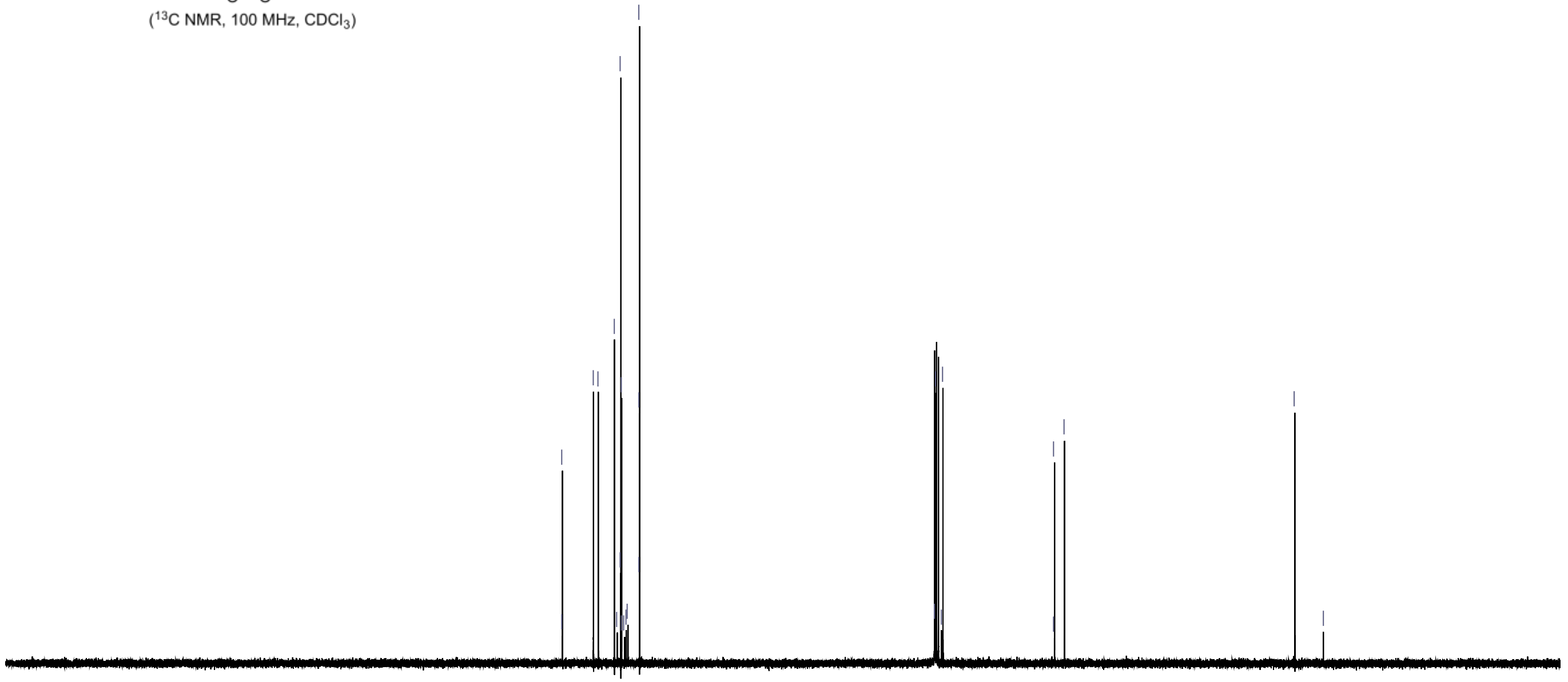




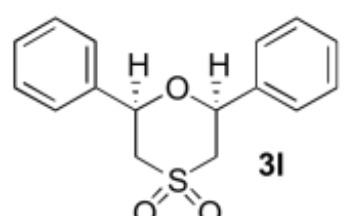

( ${ }^{1} \mathrm{H} \mathrm{NMR}, 400 \mathrm{MHz}, \mathrm{CDCl}_{3}$ )
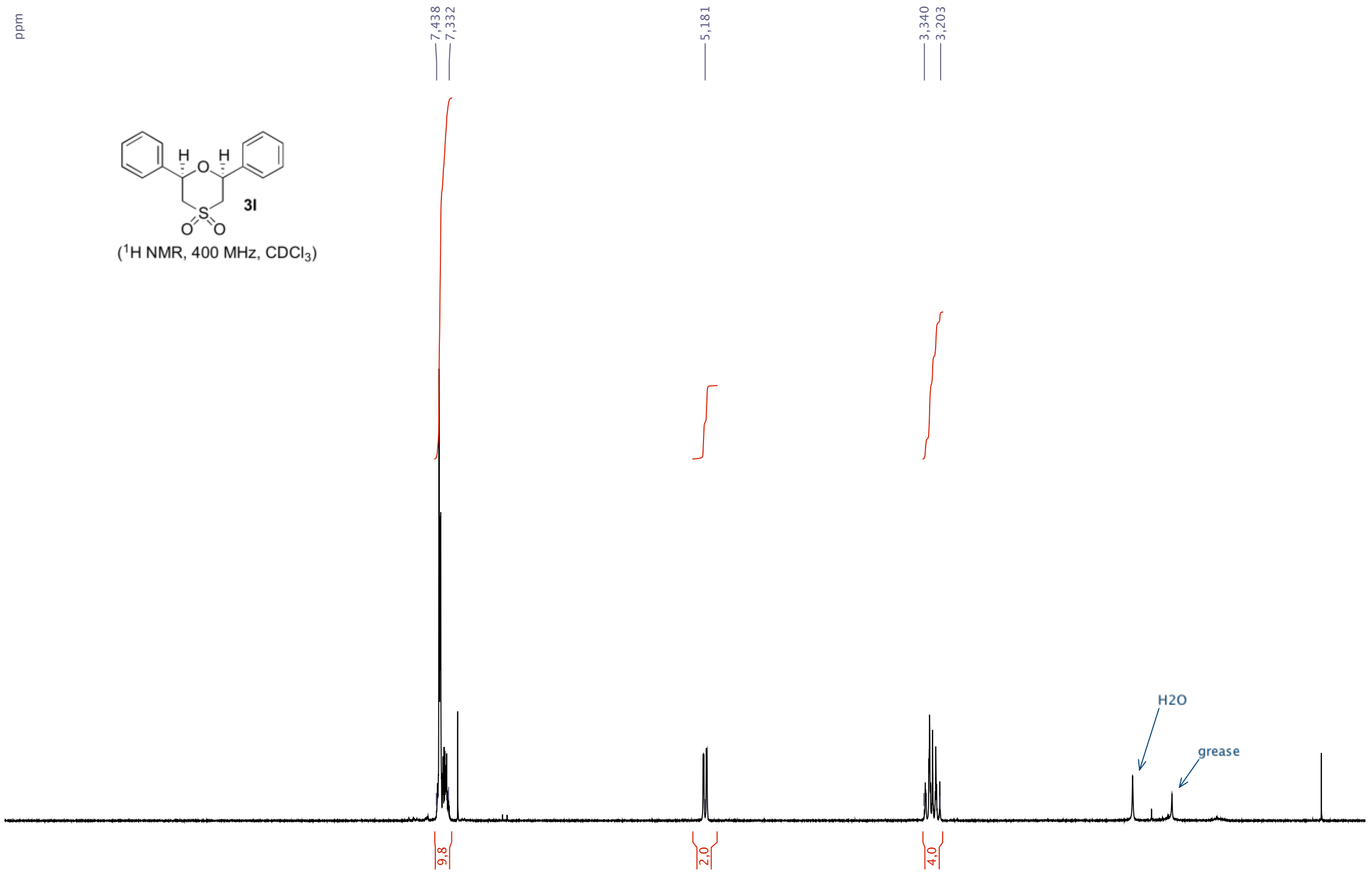


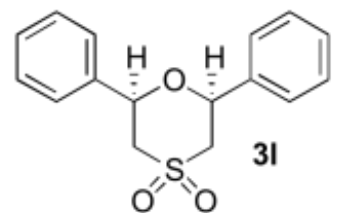

$\left({ }^{13} \mathrm{C}\right.$ NMR, $\left.100 \mathrm{MHz}, \mathrm{CDCl}_{3}\right)$ 


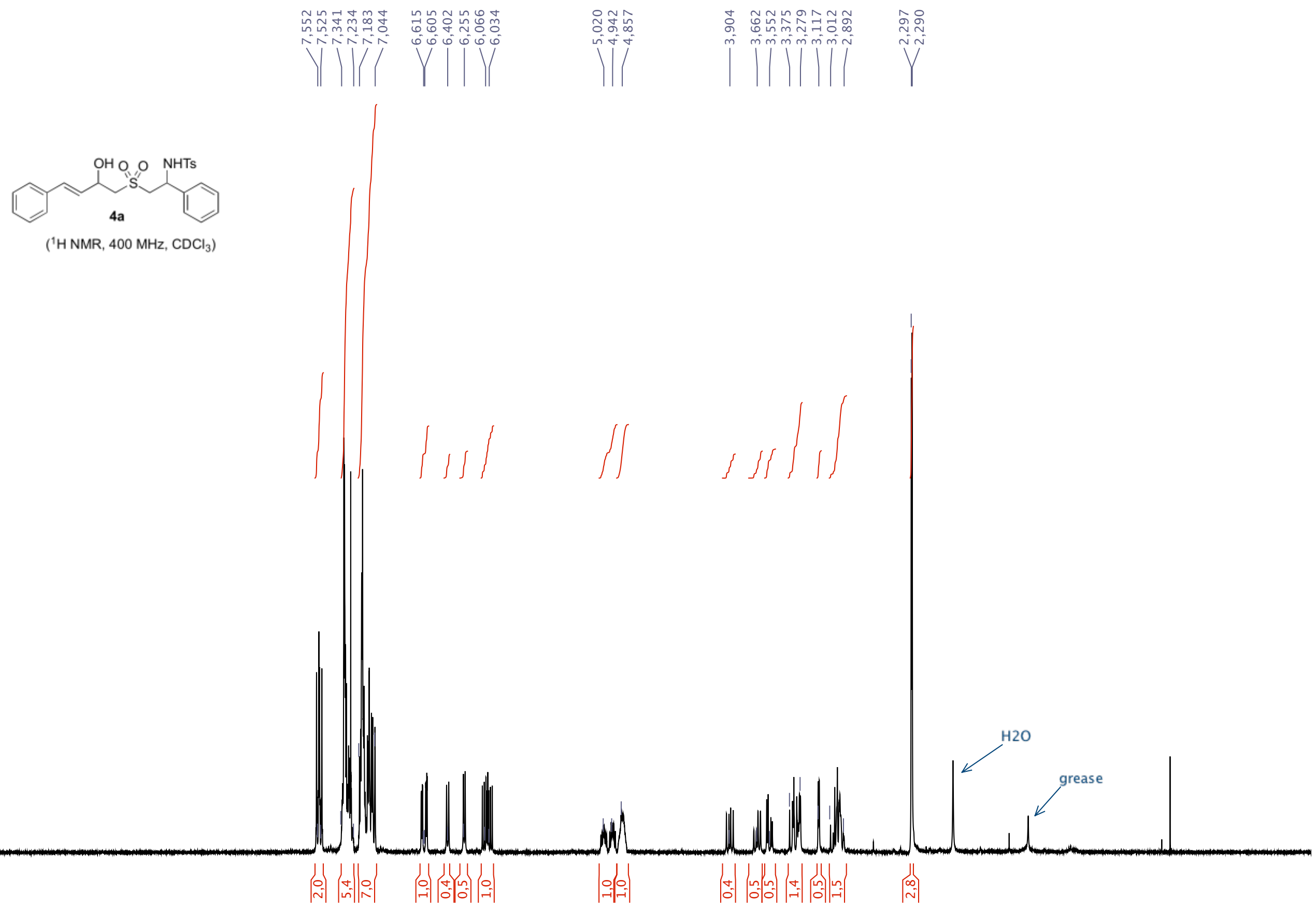


$\mathrm{OH} O \mathrm{O}$ NHTs

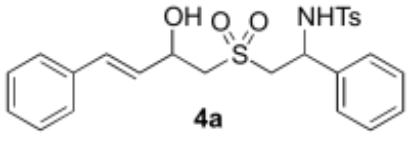

$\left({ }^{13} \mathrm{C} \mathrm{NMR}, 100 \mathrm{MHz}, \mathrm{CDCl}_{3}\right)$

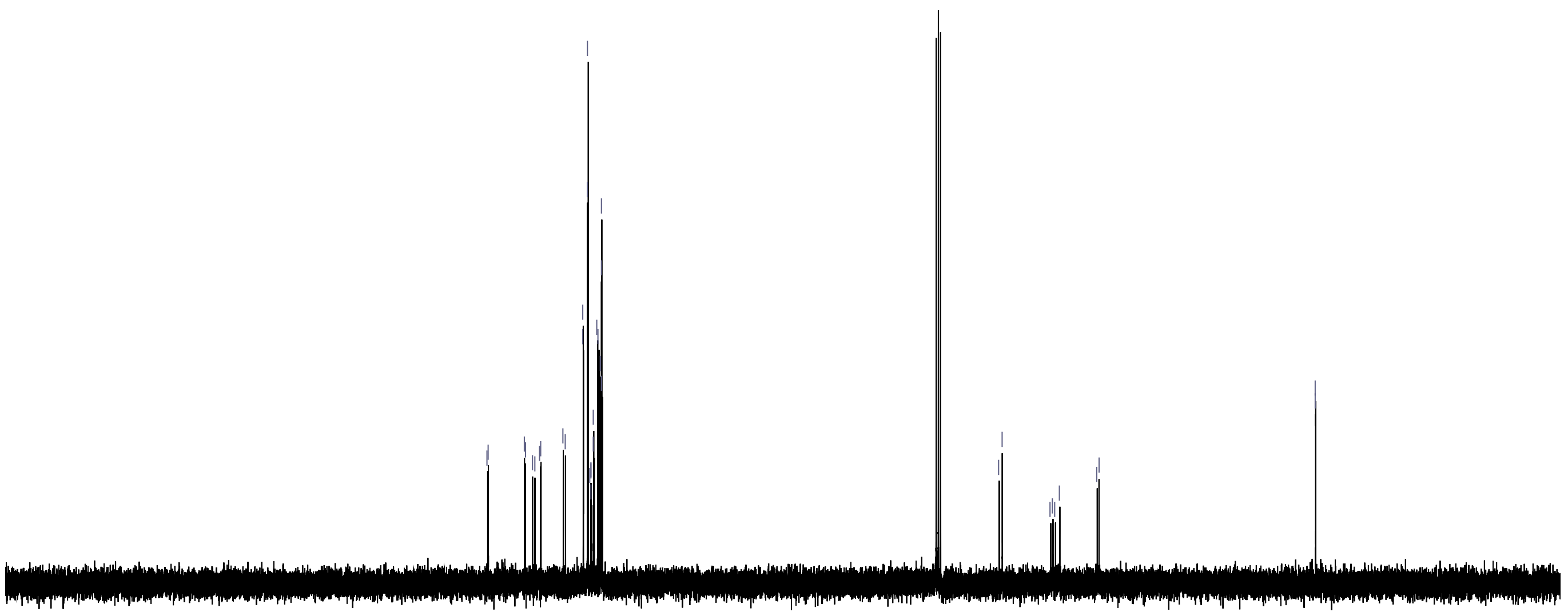




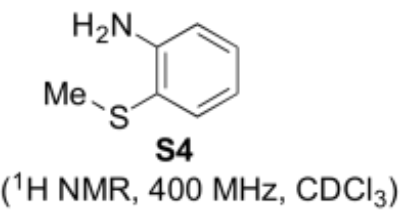

( ${ }^{1} \mathrm{H}$ NMR, $400 \mathrm{MHz}, \mathrm{CDCl}_{3}$ )
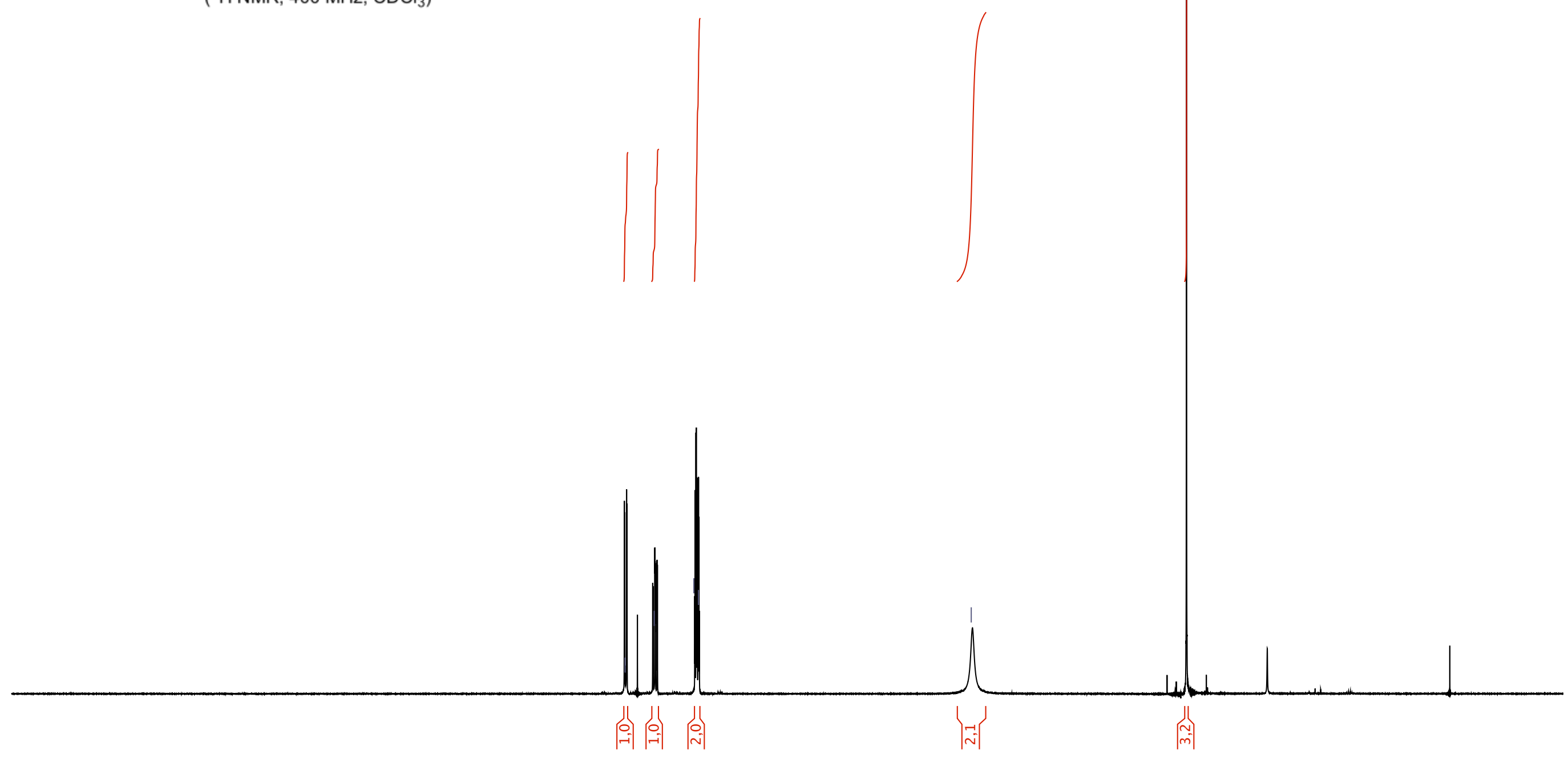


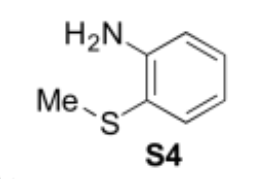

$\left({ }^{13} \mathrm{C} \mathrm{NMR}, 100 \mathrm{MHz}, \mathrm{CDCl}_{3}\right)$

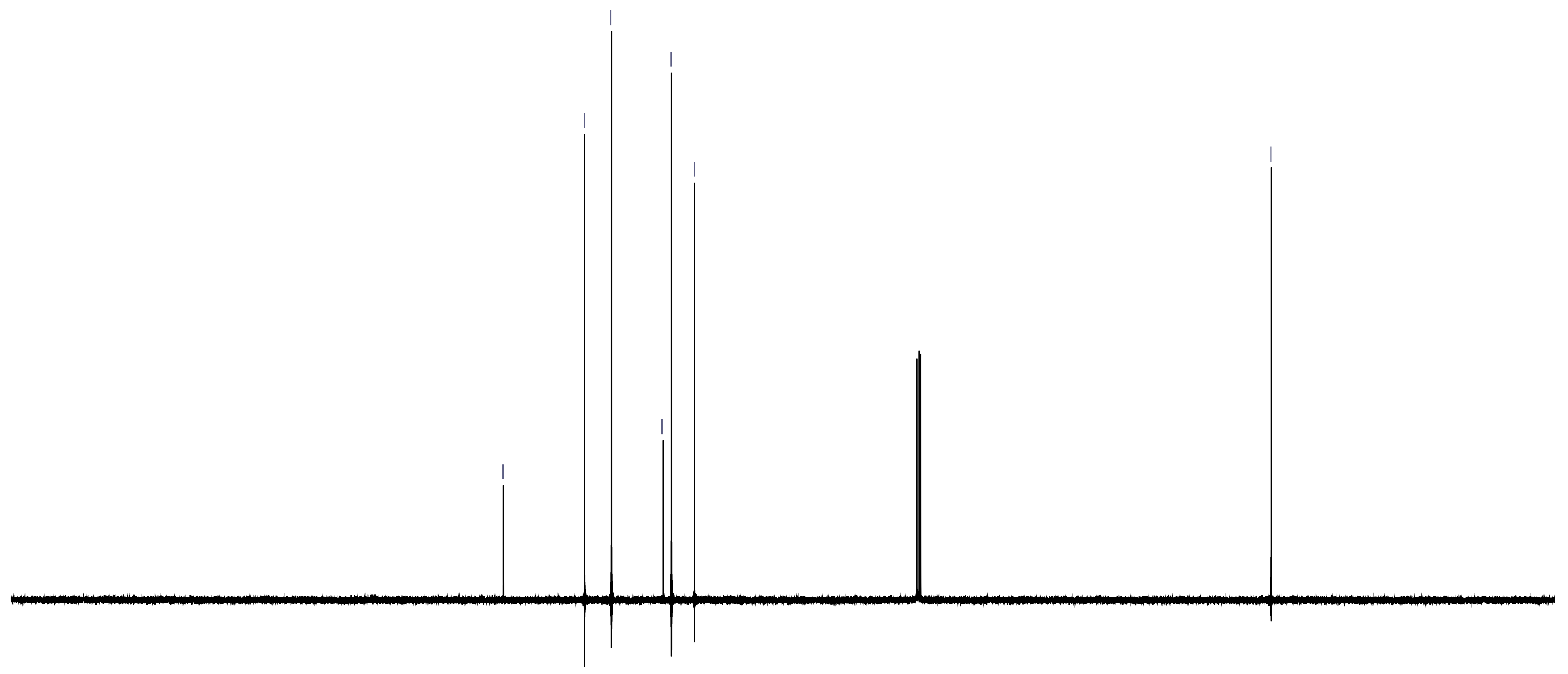




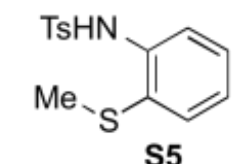

( ${ }^{1} \mathrm{H} \mathrm{NMR,}, 400 \mathrm{MHz}, \mathrm{CDCl}_{3}$ )
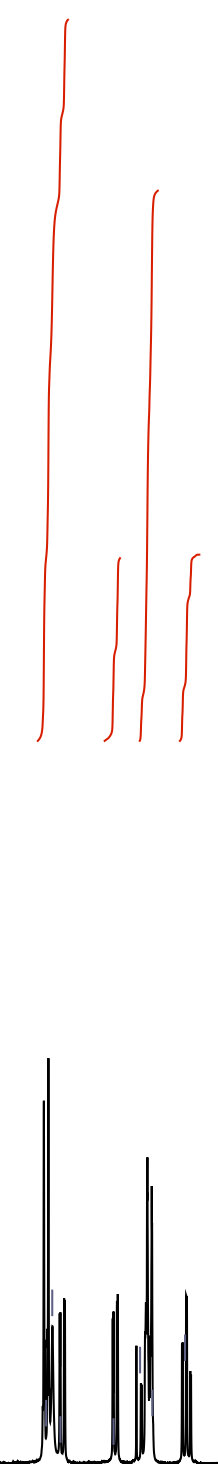

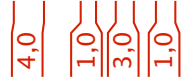
夙夙 


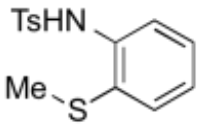

S5

$\left({ }^{13} \mathrm{C} \mathrm{NMR}, 100 \mathrm{MHz}, \mathrm{CDCl}_{3}\right)$ 

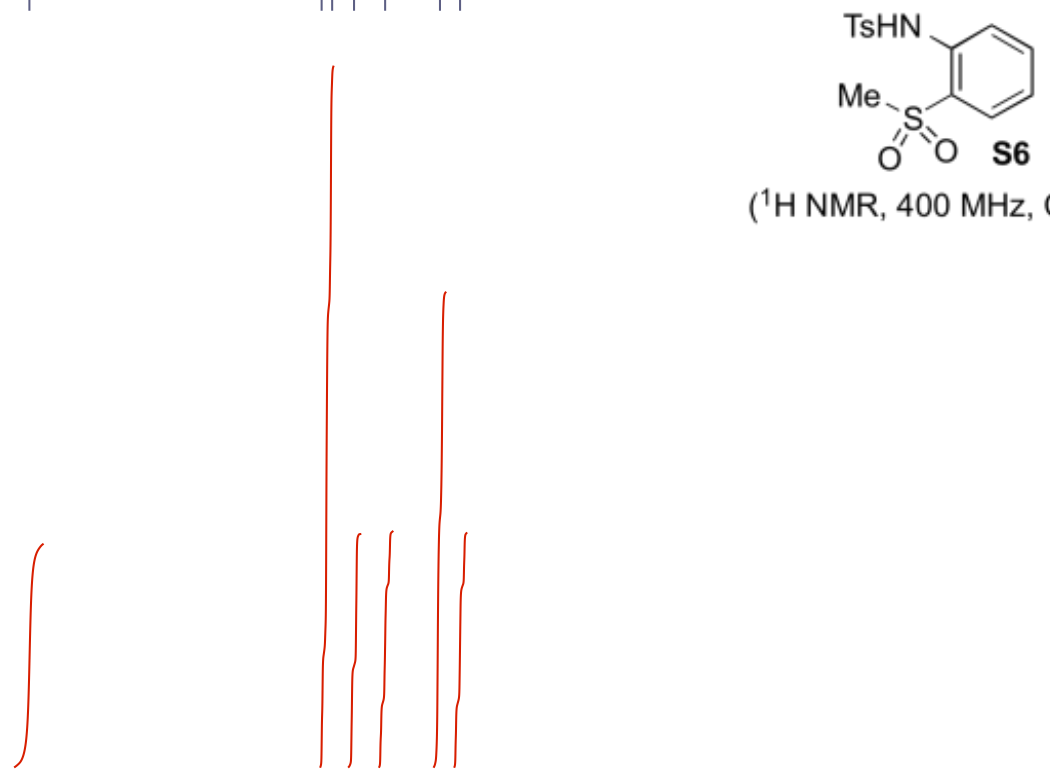

$\left({ }^{1} \mathrm{H} \mathrm{NMR}, 400 \mathrm{MHz}, \mathrm{CDCl}_{3}\right.$ )

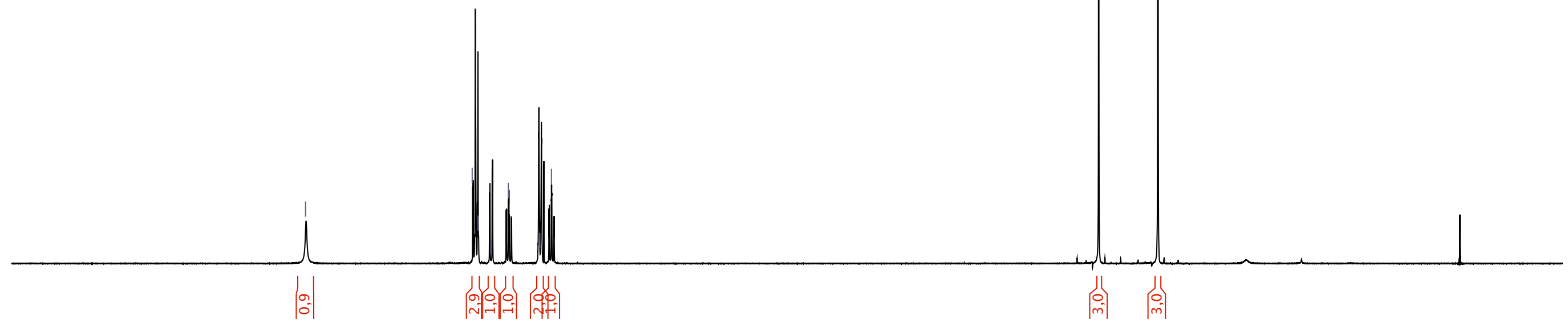


TsHN<smiles>[Y5]S(=O)(=O)c1ccccc1</smiles>

$\left({ }^{13} \mathrm{C}\right.$ NMR, $\left.100 \mathrm{MHz}, \mathrm{CDCl}_{3}\right)$

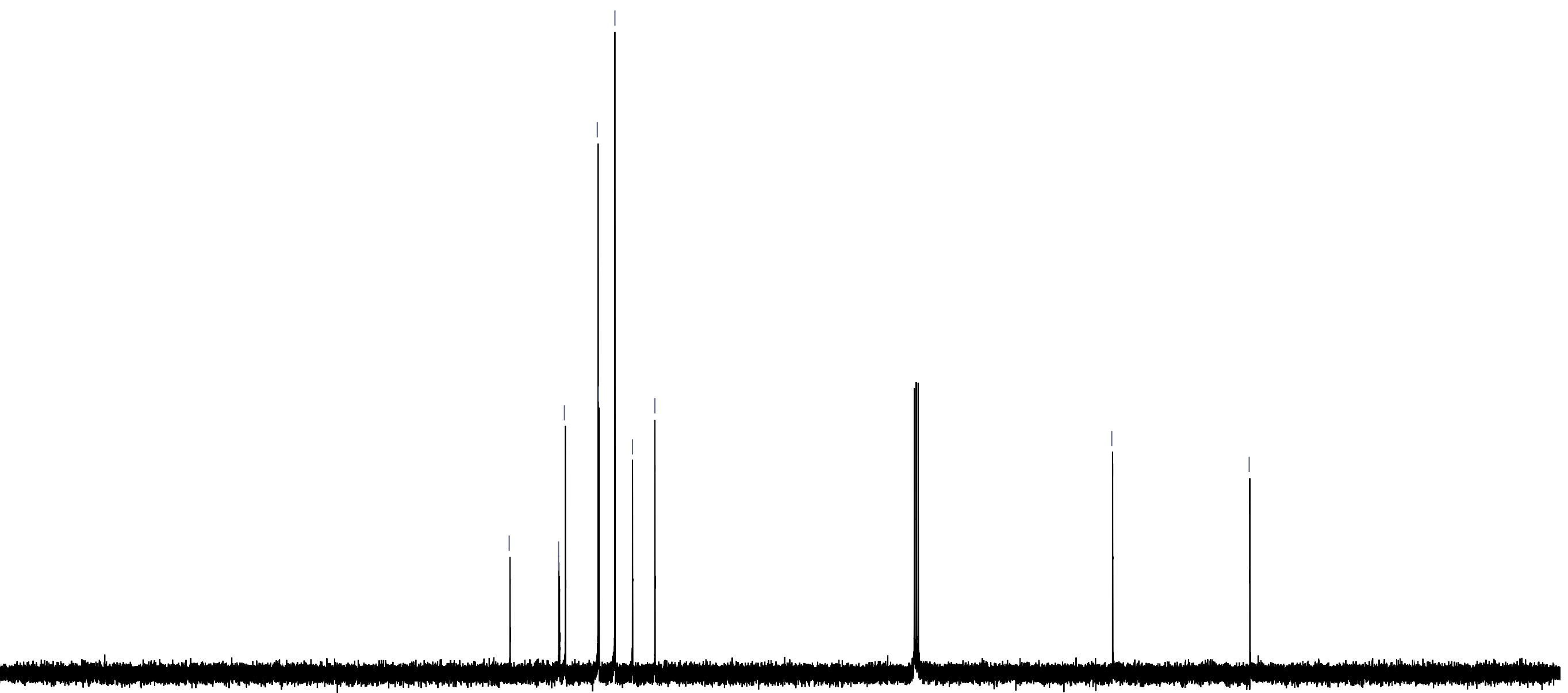




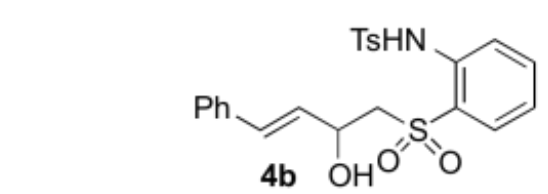

( ${ }^{1} \mathrm{H} \mathrm{NMR}, 400 \mathrm{MHz}, \mathrm{CDCl}_{3}$ )

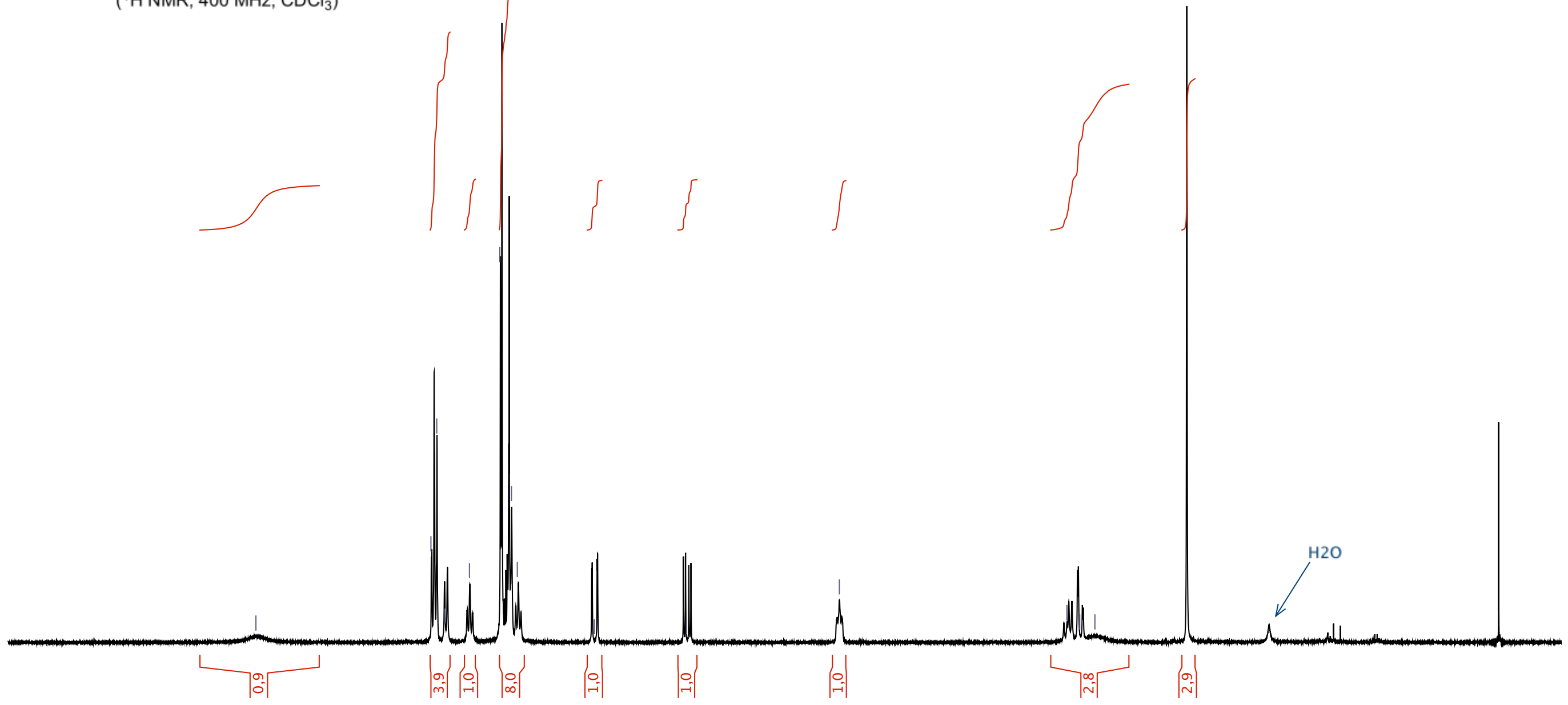




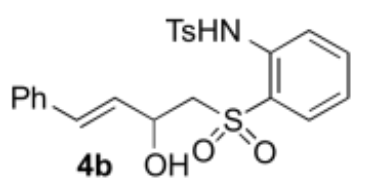

$\left({ }^{13} \mathrm{C} \mathrm{NMR,}, 100 \mathrm{MHz}, \mathrm{CDCl}_{3}\right)$

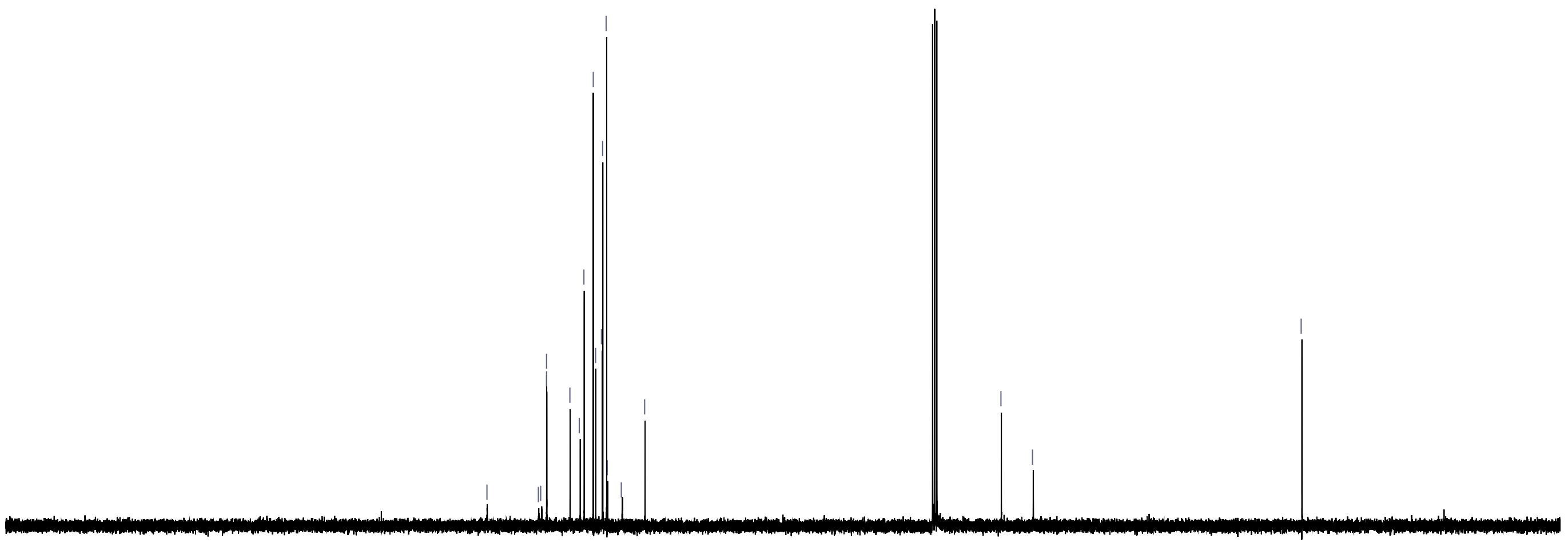




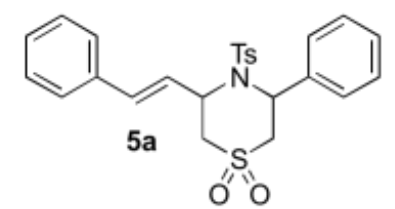

$\left({ }^{1} \mathrm{H} \mathrm{NMR}, 400 \mathrm{MHz}, \mathrm{CDCl}_{3}\right.$ )
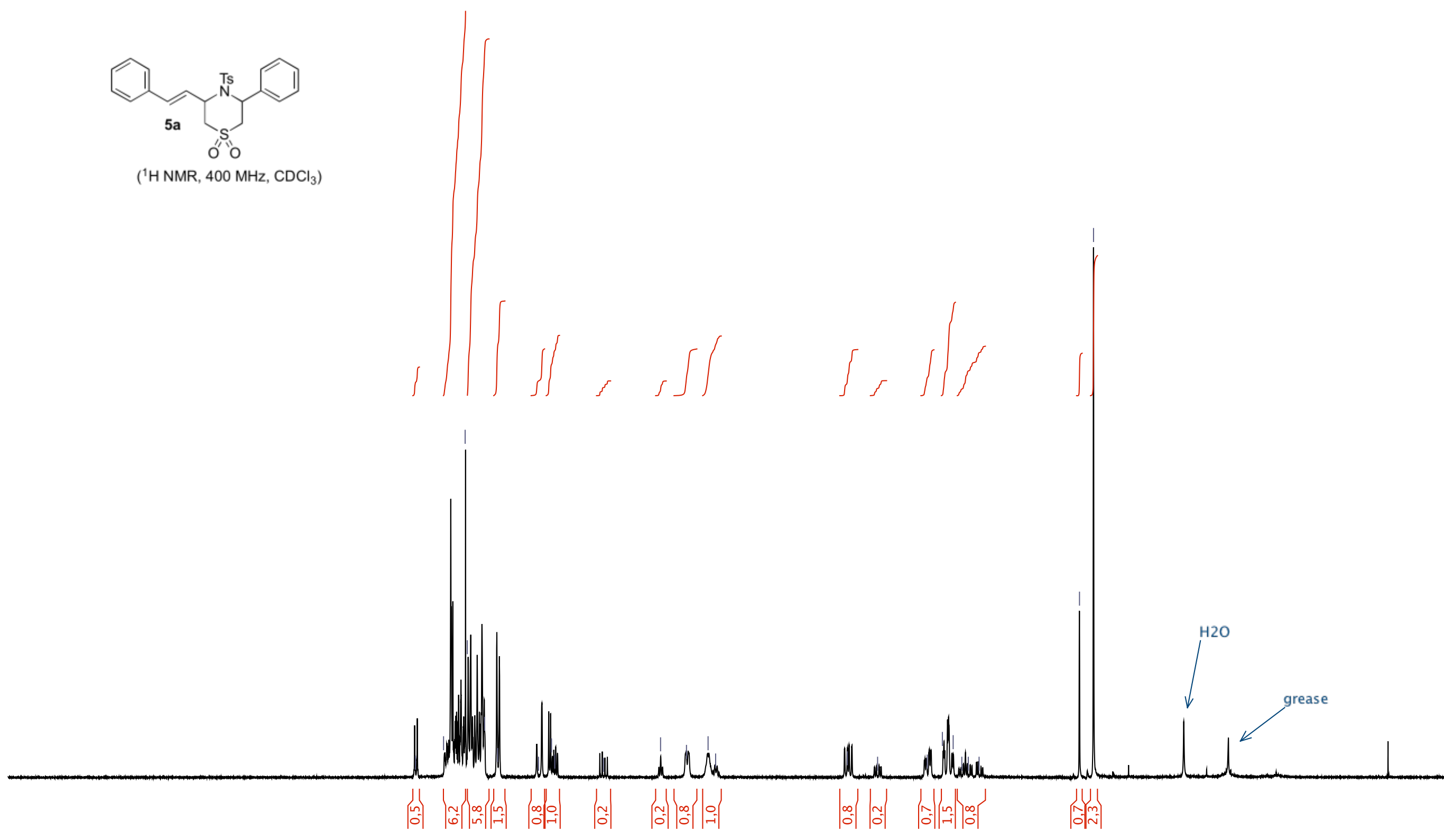


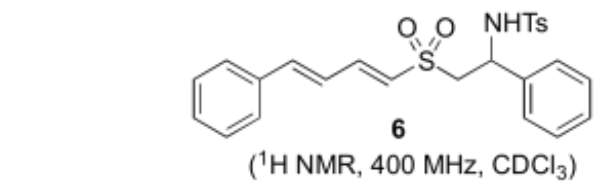

( ${ }^{1} \mathrm{H} \mathrm{NMR}, 400 \mathrm{MHz}, \mathrm{CDCl}_{3}$ )
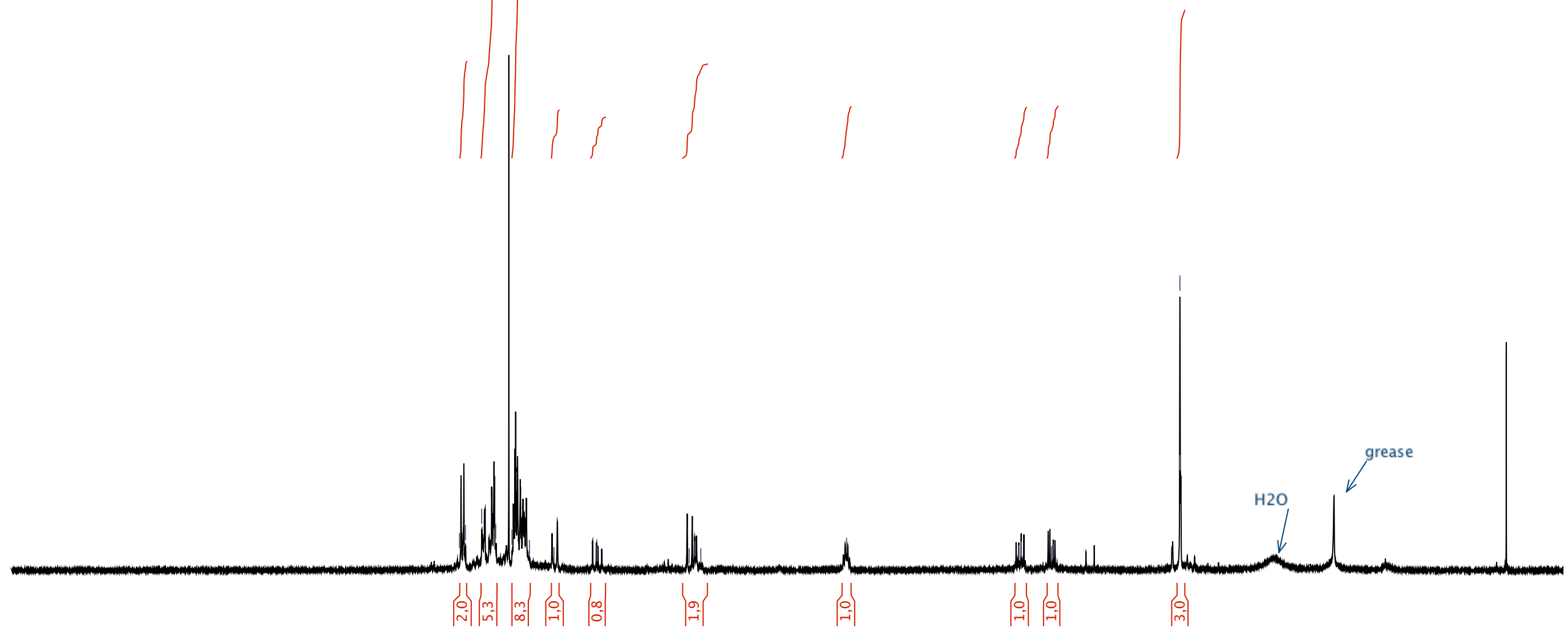
흘
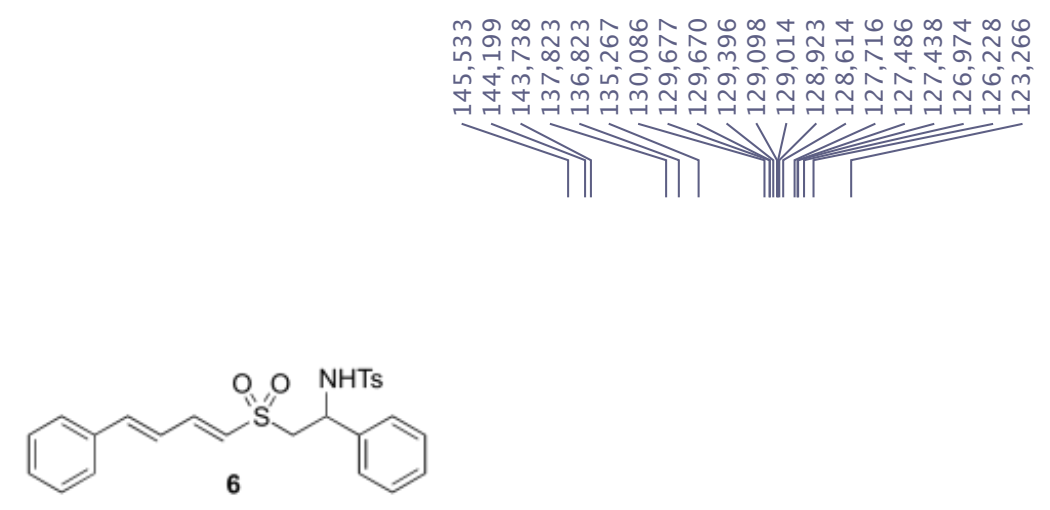

$\left({ }^{13} \mathrm{C} \mathrm{NMR}, 100 \mathrm{MHz}, \mathrm{CDCl}_{3}\right)$

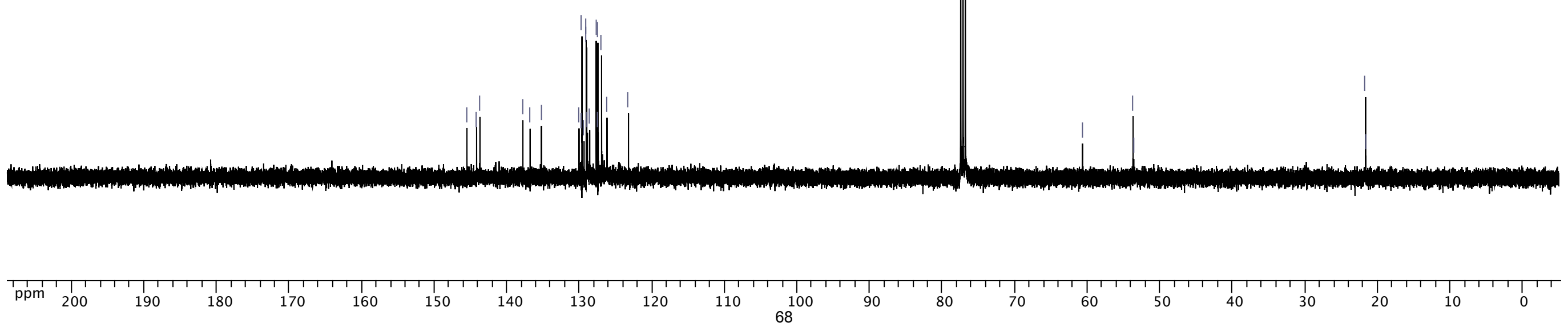




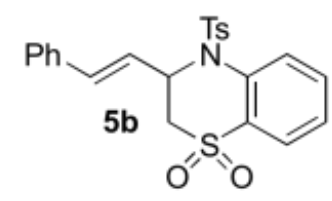

( ${ }^{1} \mathrm{H} \mathrm{NMR}, 400 \mathrm{MHz}, \mathrm{CDCl}_{3}$ )

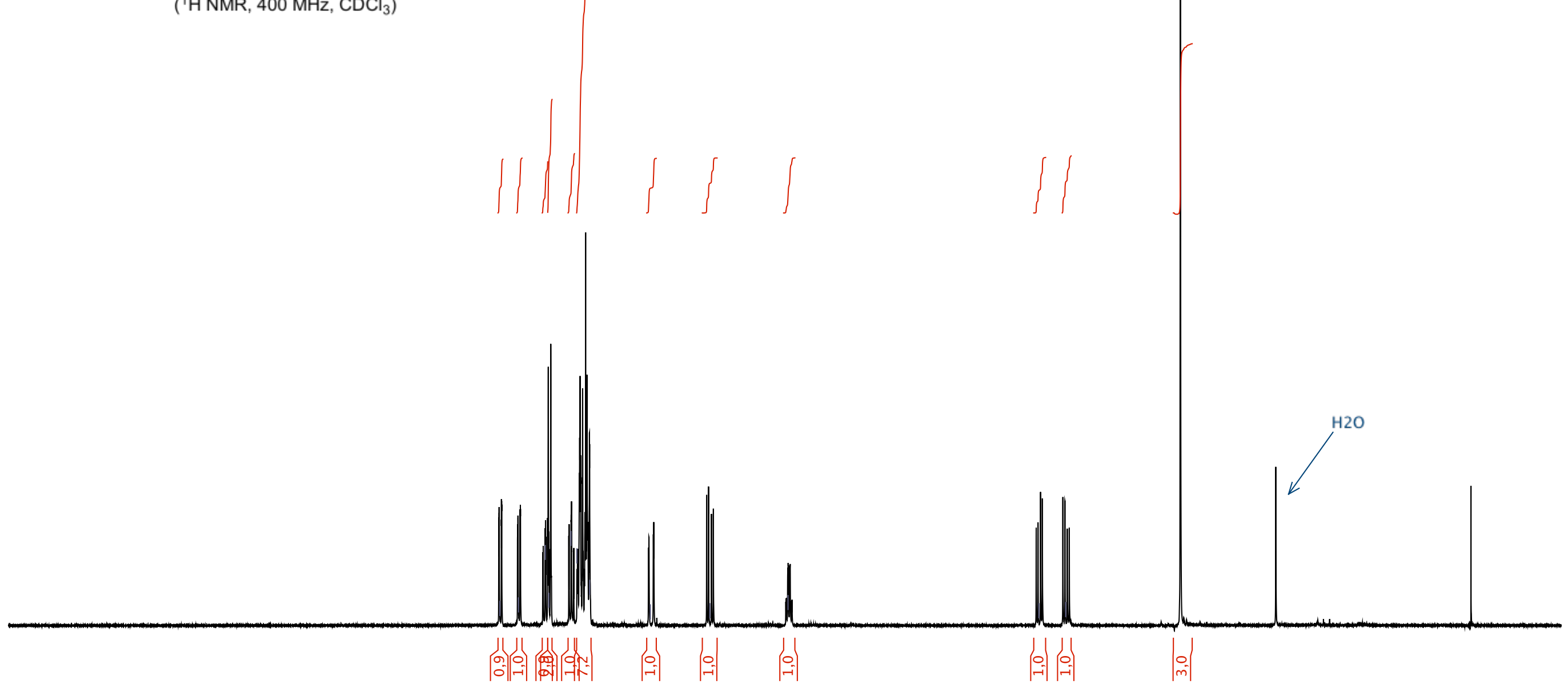




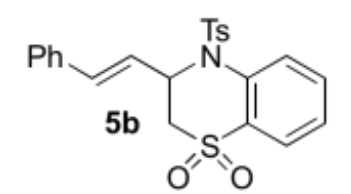

$\left({ }^{13} \mathrm{C} \mathrm{NMR}, 100 \mathrm{MHz}, \mathrm{CDCl}_{3}\right)$

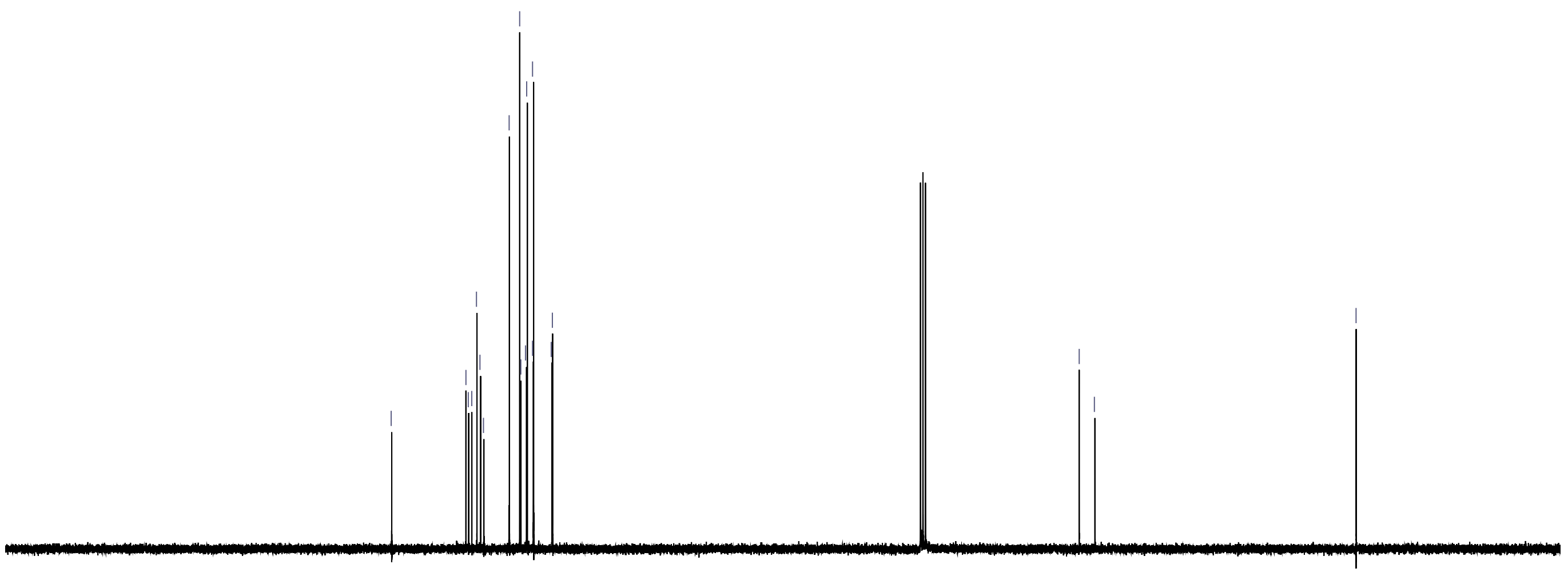




$$
\overbrace{\mathbf{S} 7}^{\mathrm{OH}}
$$

$\left({ }^{1} \mathrm{H} \mathrm{NMR}, 400 \mathrm{MHz}, \mathrm{CDCl}_{3}\right.$ )

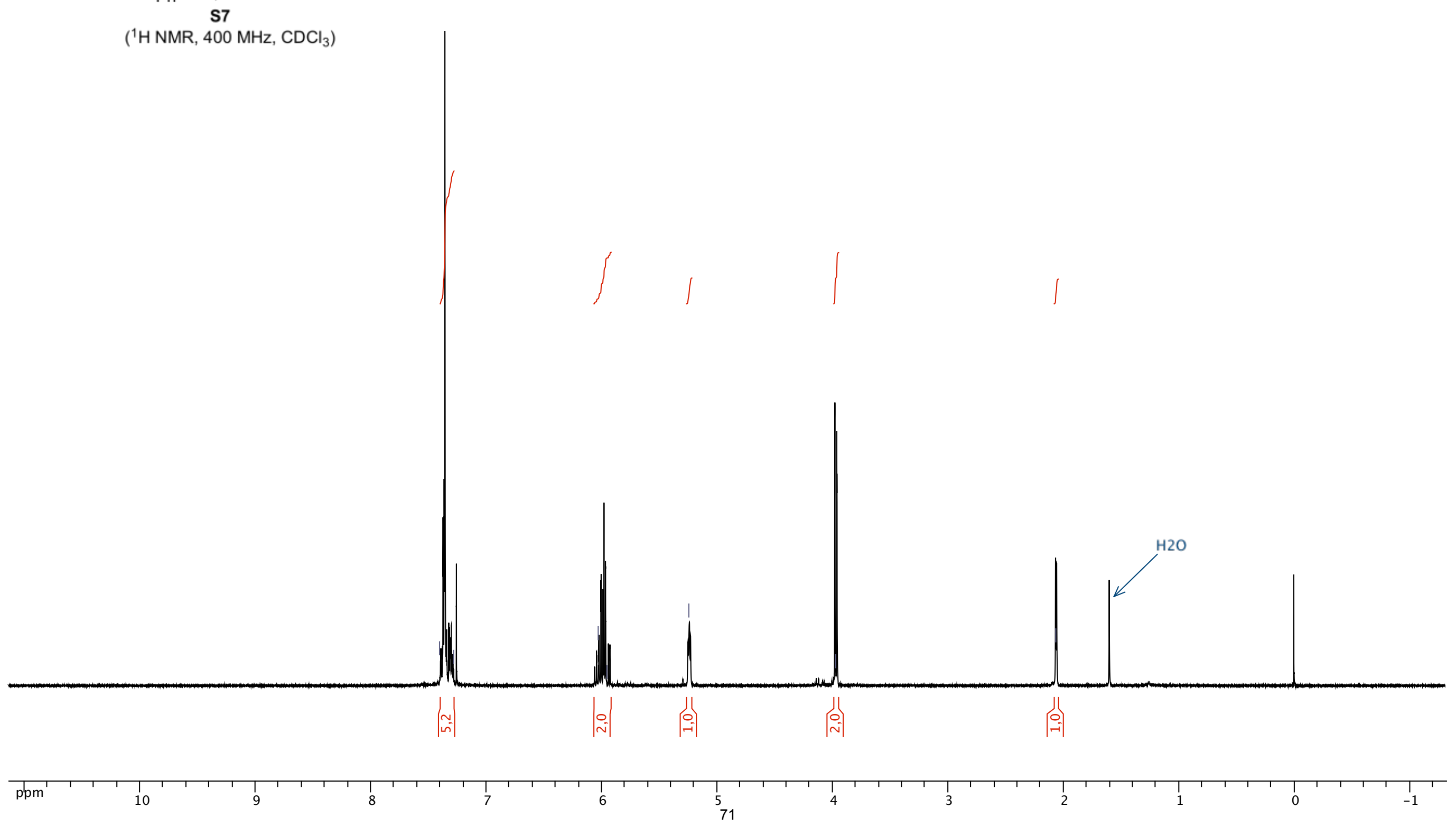




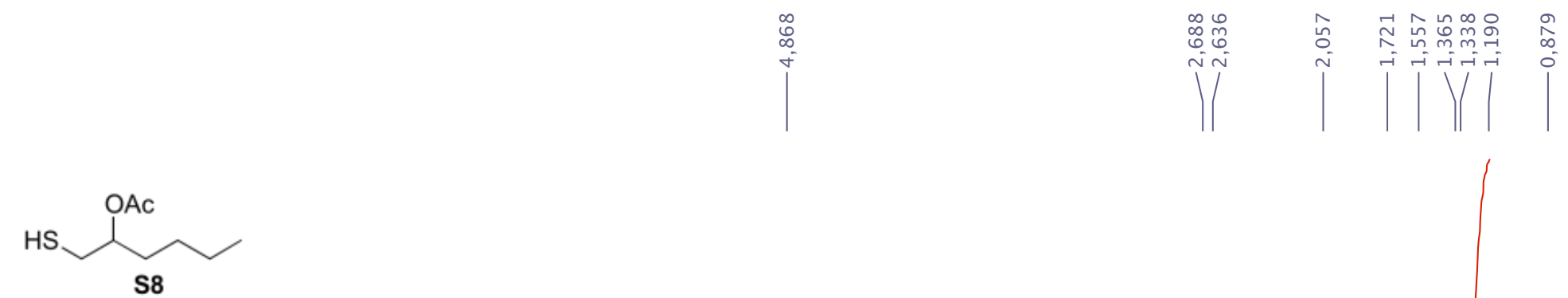

( ${ }^{1} \mathrm{H} \mathrm{NMR}, 400 \mathrm{MHz}, \mathrm{CDCl}_{3}$ )

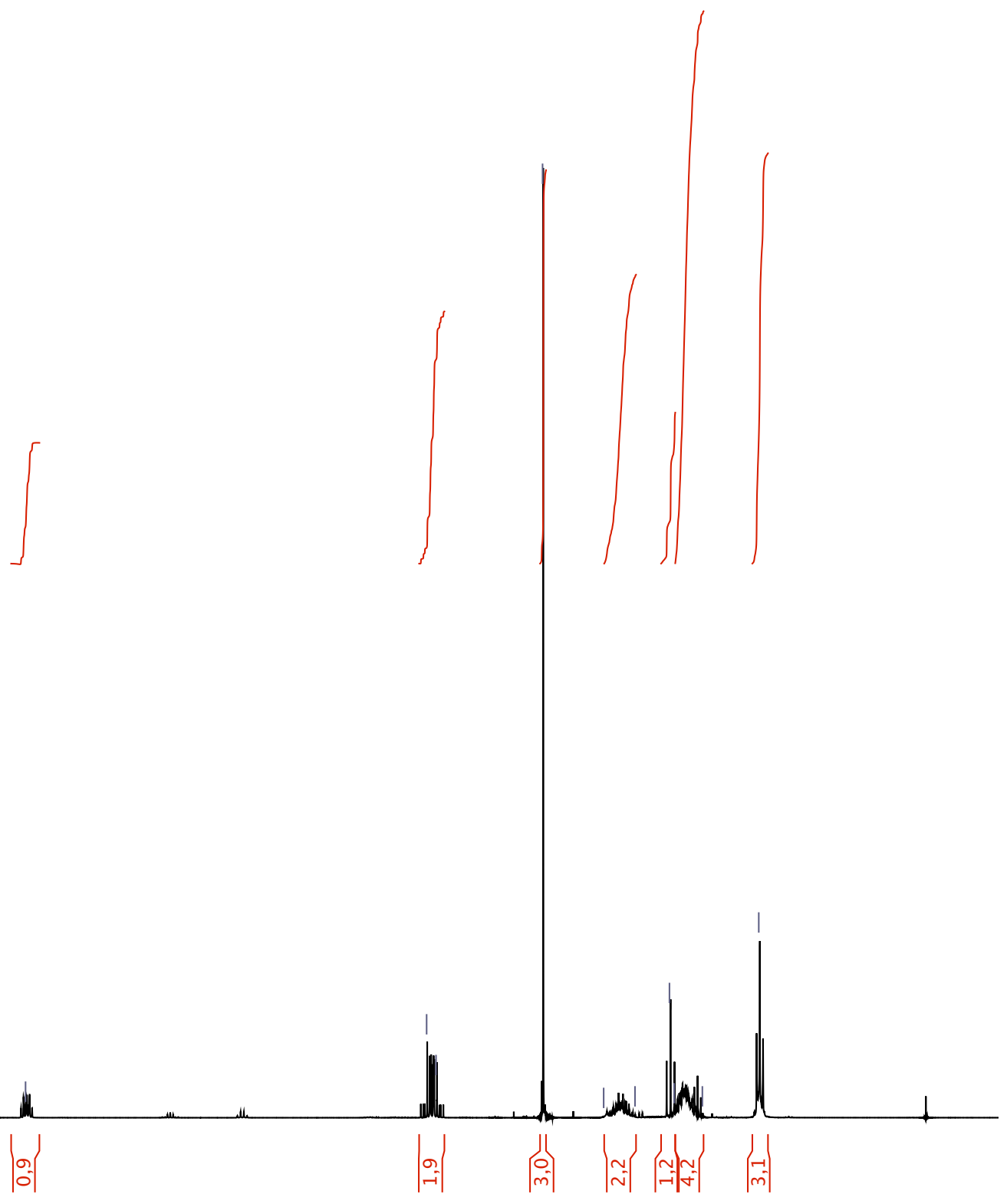




$$
{ }_{\left({ }^{13} \mathrm{C} \mathrm{NMR}, 100 \mathrm{MHz}, \mathrm{CDCl}_{3}\right)}^{\mathrm{OAC}}
$$
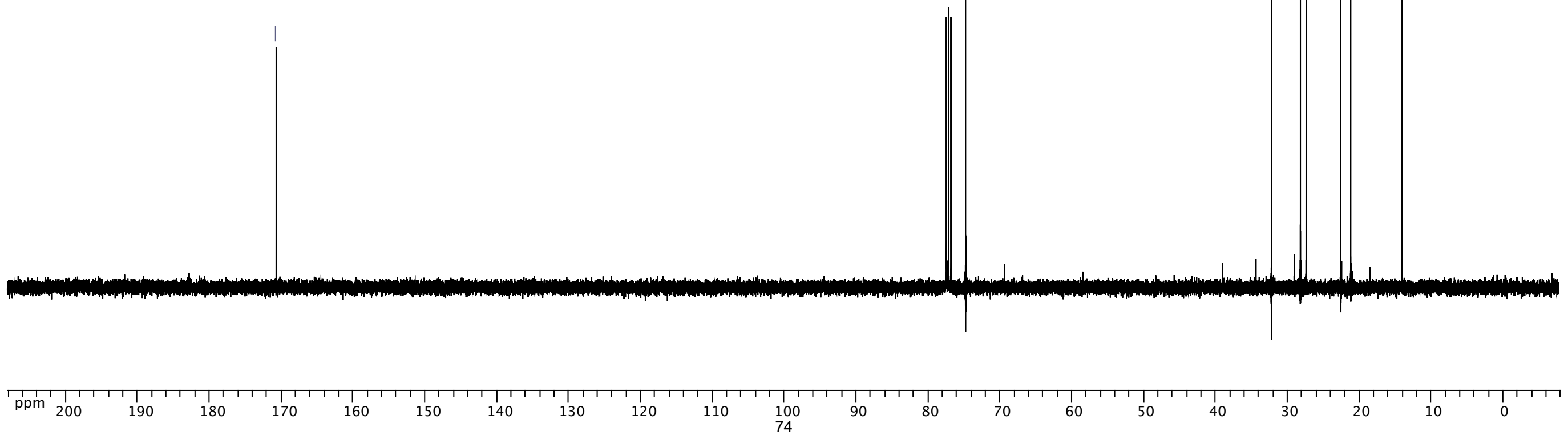


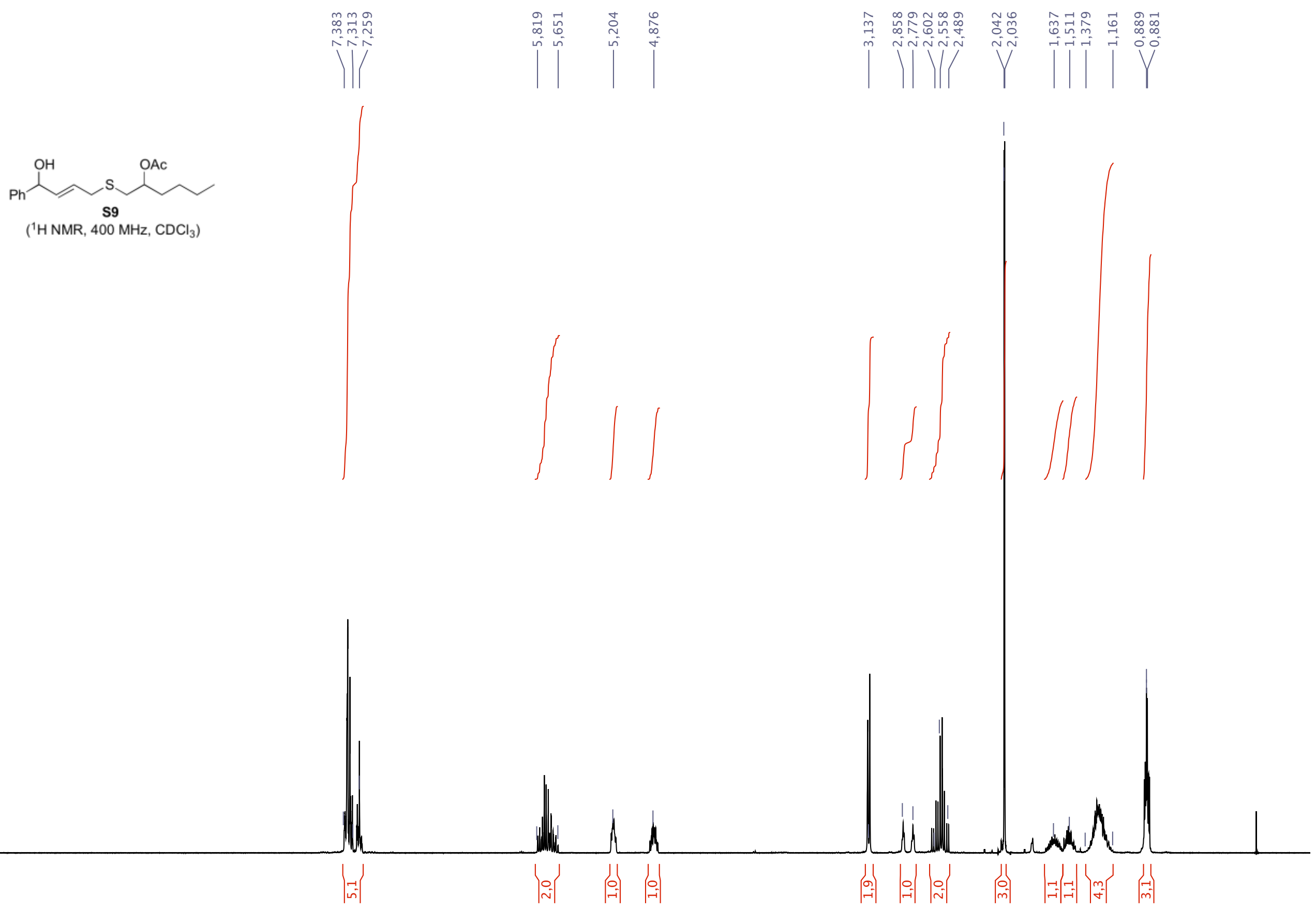




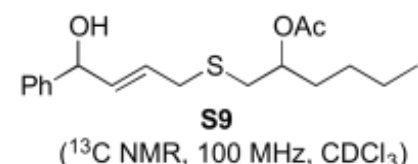

$\left({ }^{13} \mathrm{C} \mathrm{NMR}, 100 \mathrm{MHz}, \mathrm{CDCl}_{3}\right)$

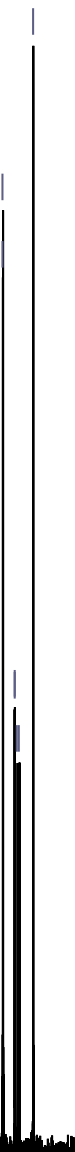



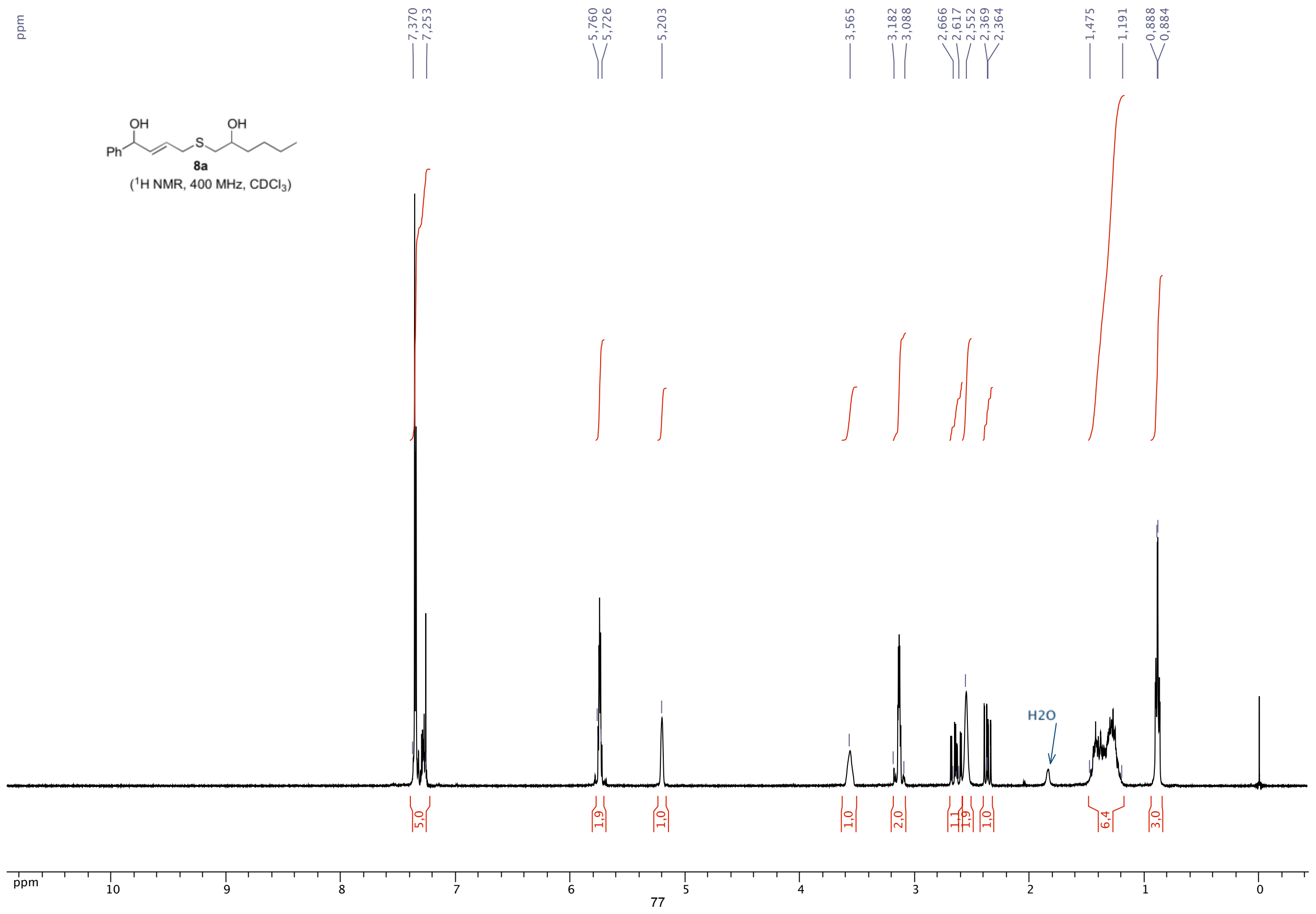

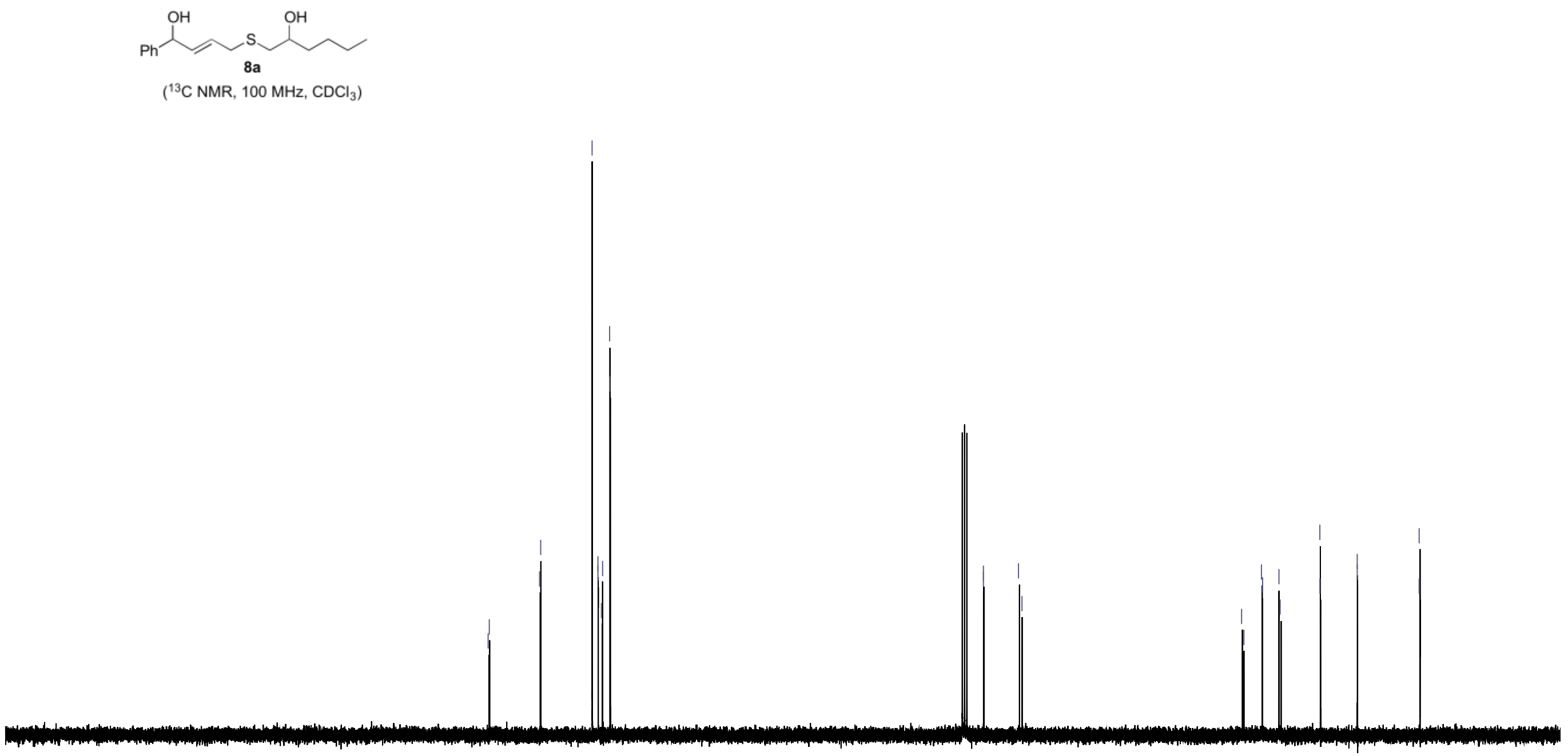


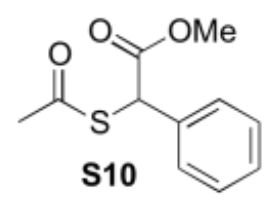

( ${ }^{1} \mathrm{H} \mathrm{NMR}, 400 \mathrm{MHz}, \mathrm{CDCl}_{3}$ )
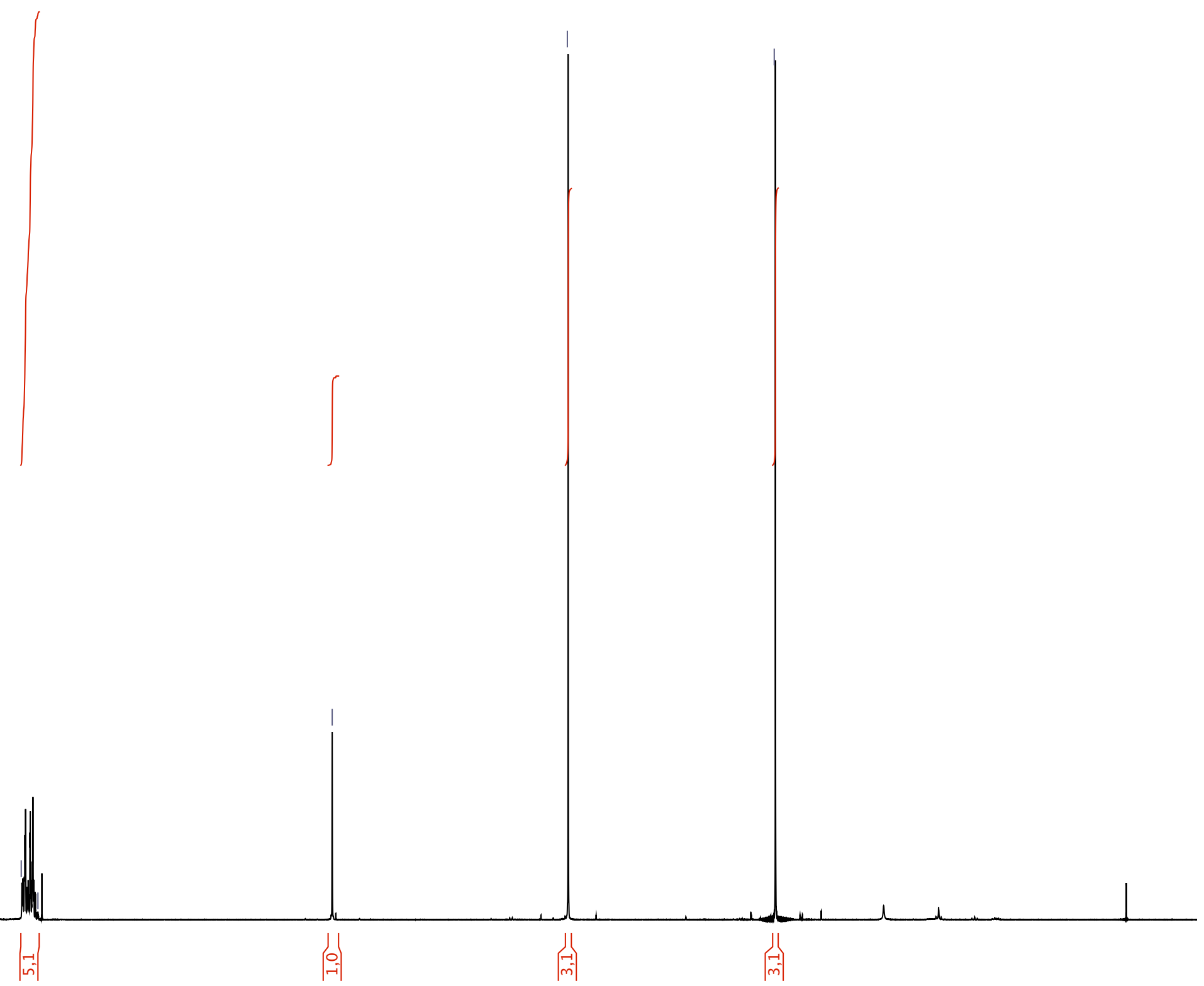


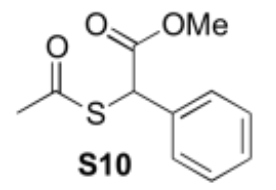

$\left({ }^{13} \mathrm{C}\right.$ NMR, $\left.100 \mathrm{MHz}, \mathrm{CDCl}_{3}\right)$

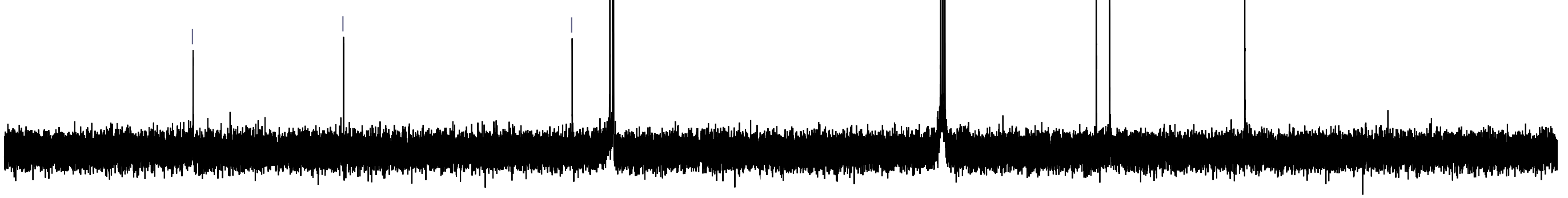


틈

$\mid$

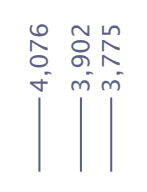

$\overbrace{\substack{\infty \\ i}}^{\substack{n \\ i}}$
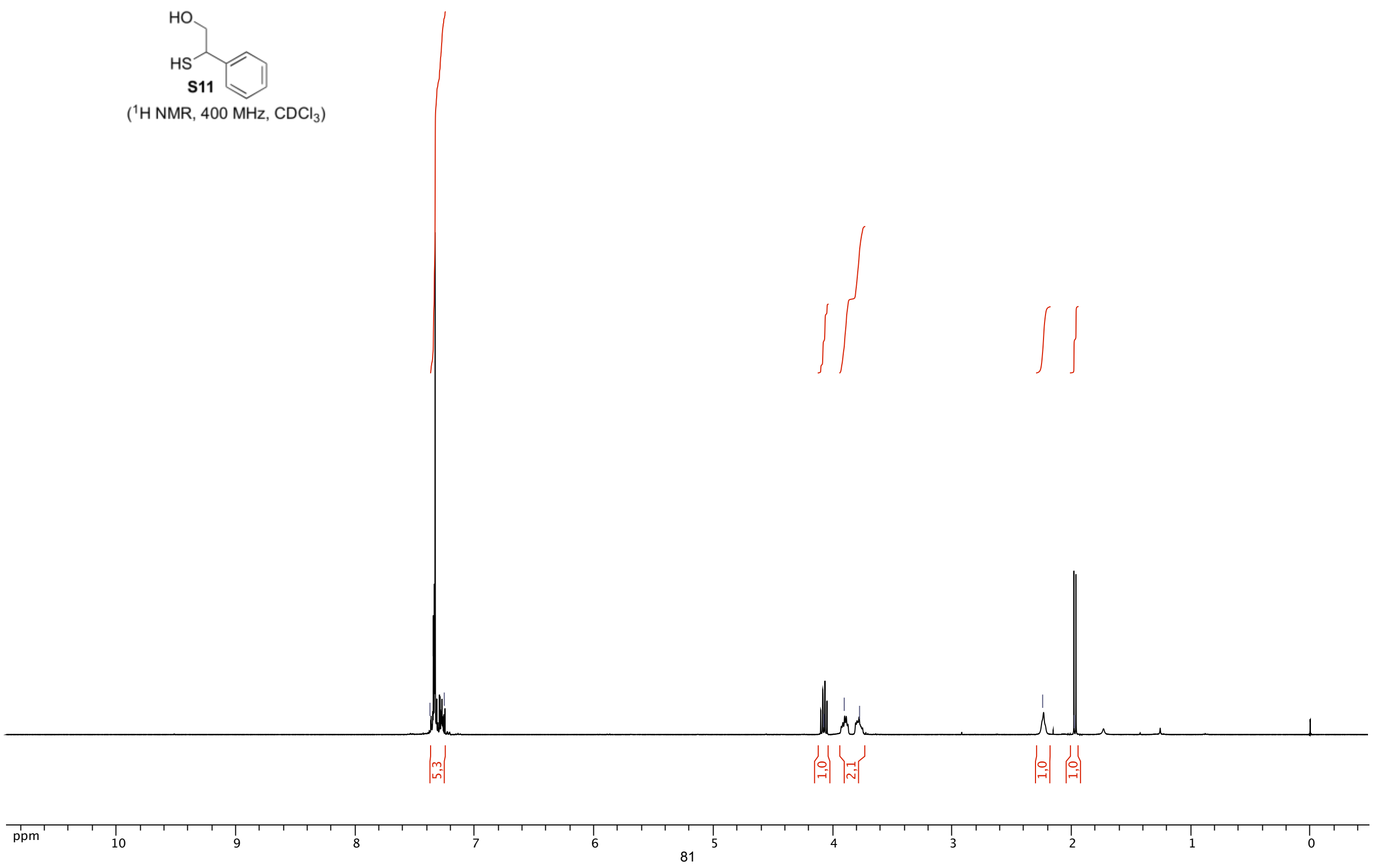


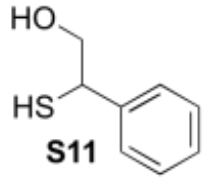

$\left({ }^{13} \mathrm{C} \mathrm{NMR}, 100 \mathrm{MHz}, \mathrm{CDCl}_{3}\right)$

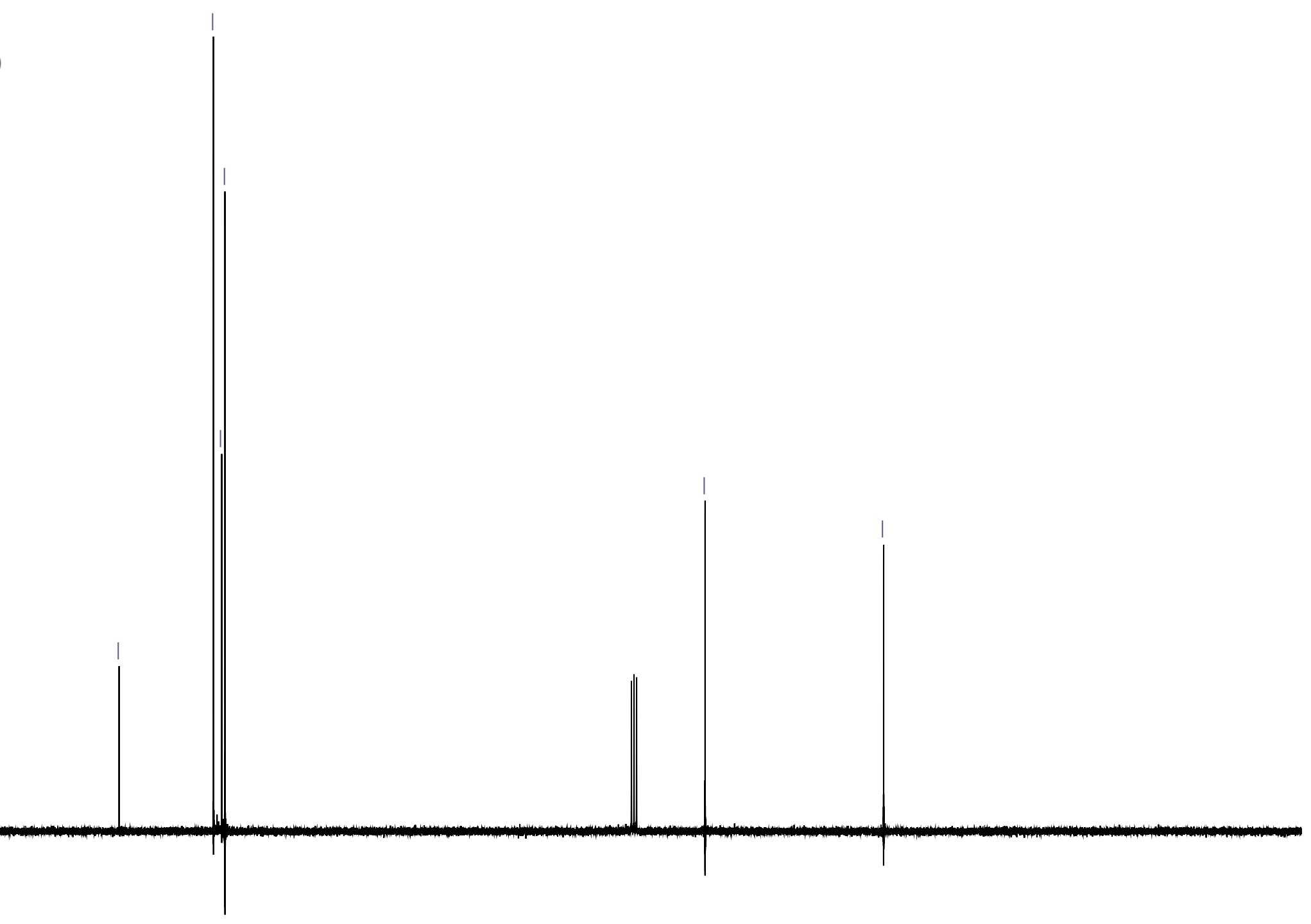




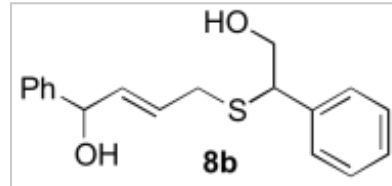

$\left({ }^{1} \mathrm{H} \mathrm{NMR}, 400 \mathrm{MHz}, \mathrm{CDCl}_{3}\right)$
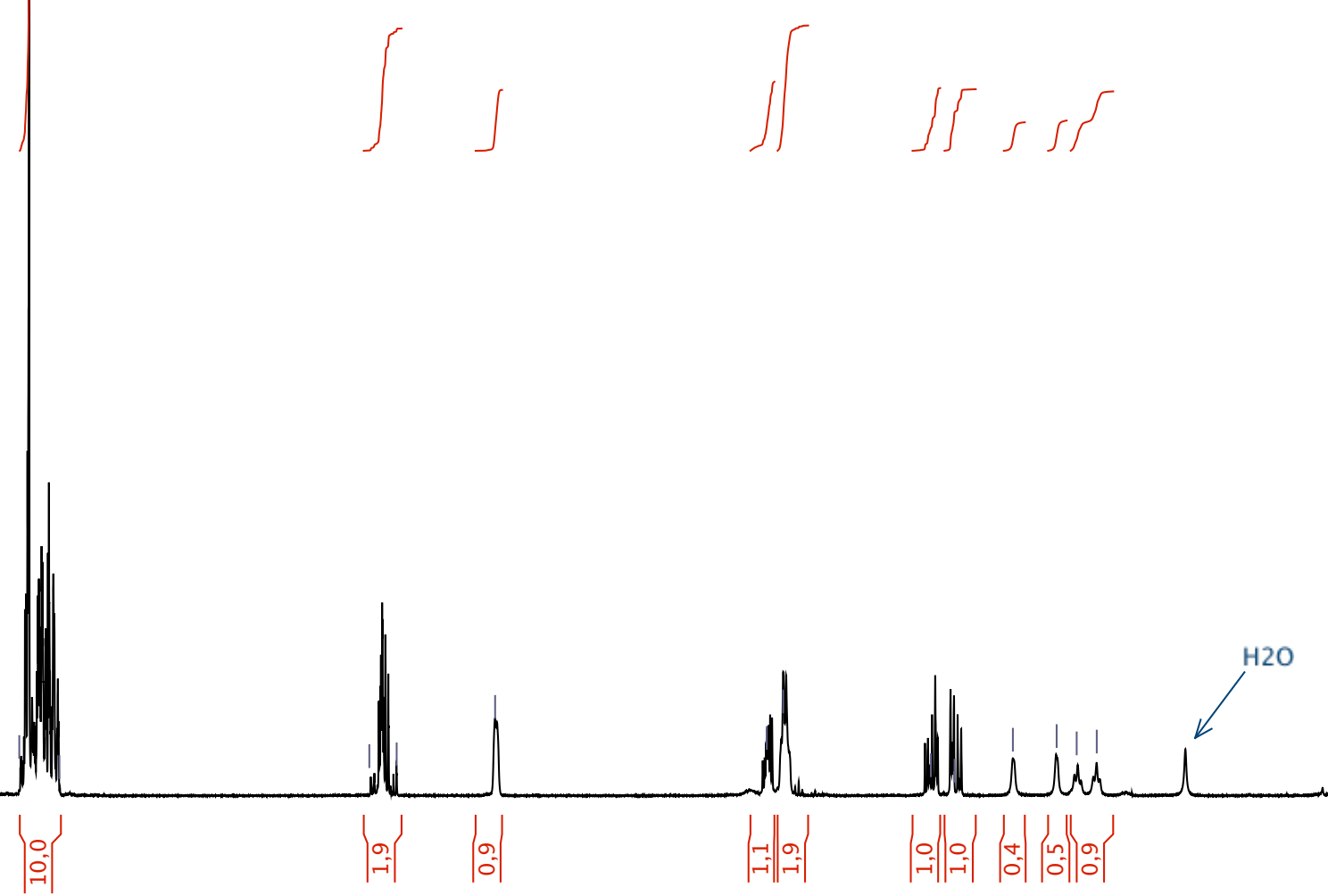


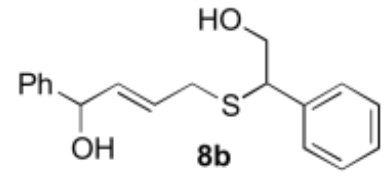

$\left({ }^{13} \mathrm{C} \mathrm{NMR}, 100 \mathrm{MHz}, \mathrm{CDCl}_{3}\right)$

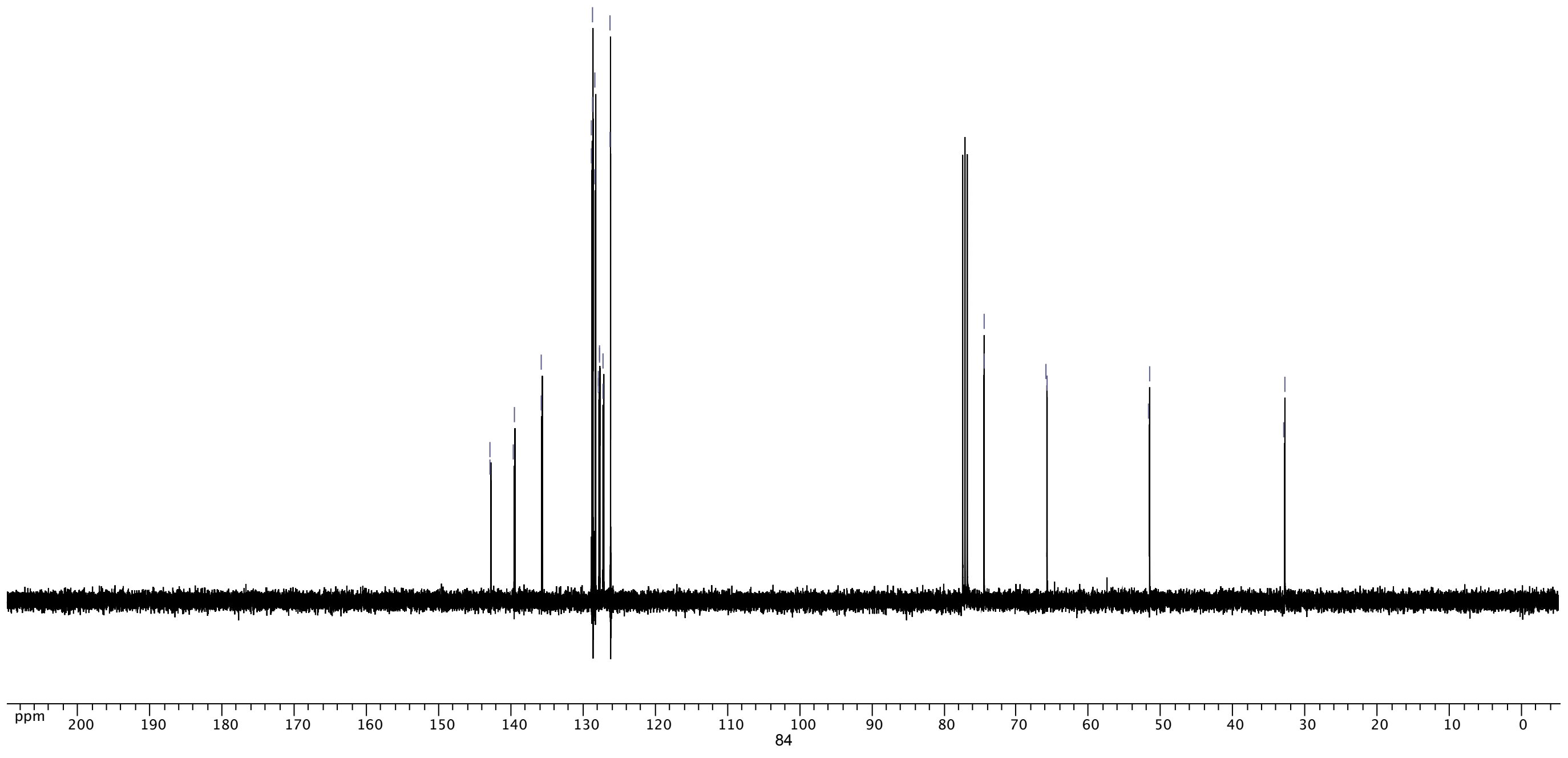




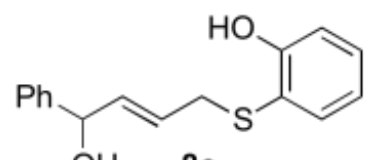

$\mathrm{OH} \quad 8 \mathrm{c}$

( ${ }^{1} \mathrm{H} \mathrm{NMR}, 400 \mathrm{MHz}, \mathrm{CDCl}_{3}$ )

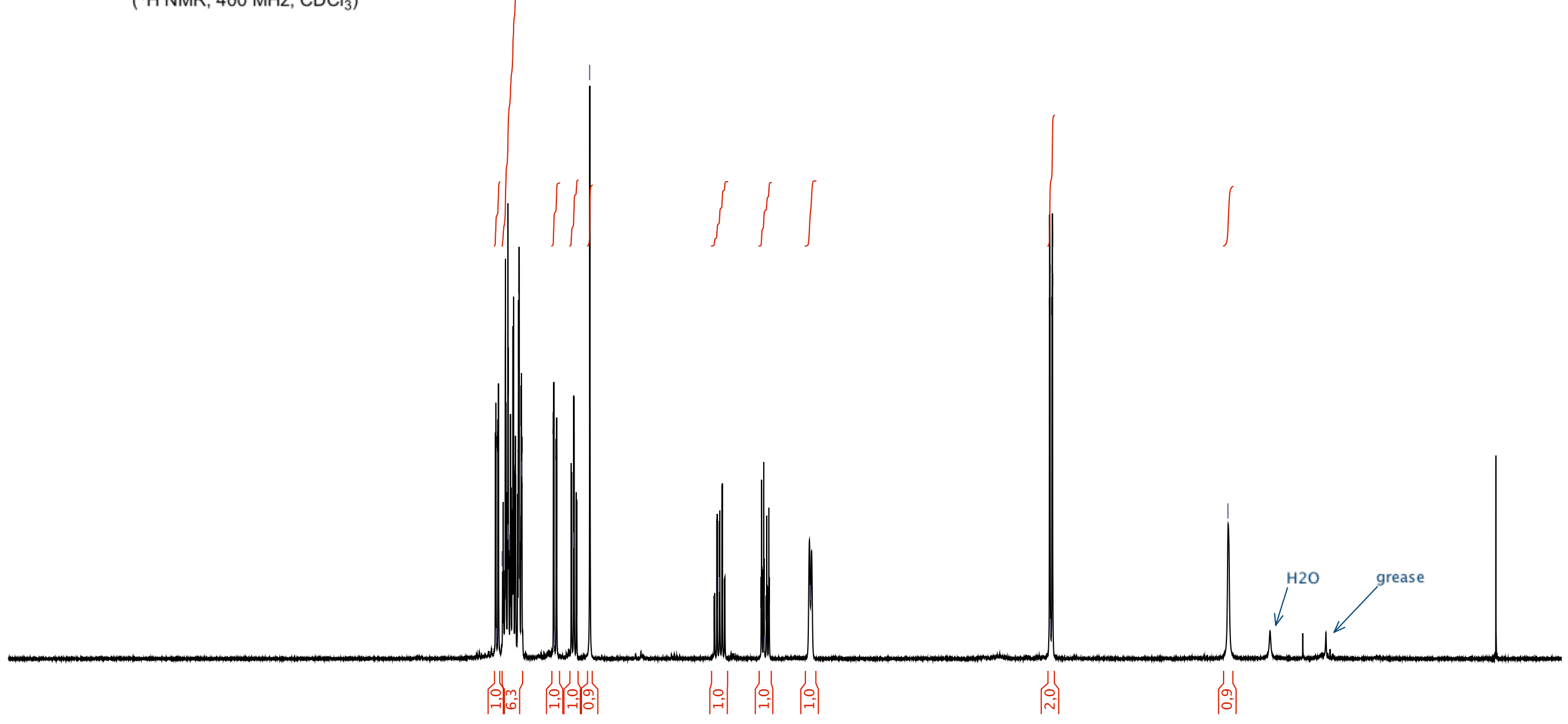




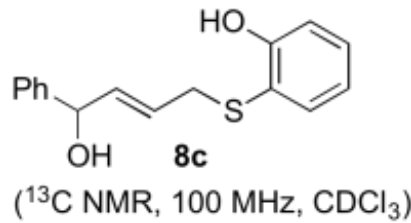

$\left({ }^{13} \mathrm{C} \mathrm{NMR}, 100 \mathrm{MHz}, \mathrm{CDCl}_{3}\right)$ 

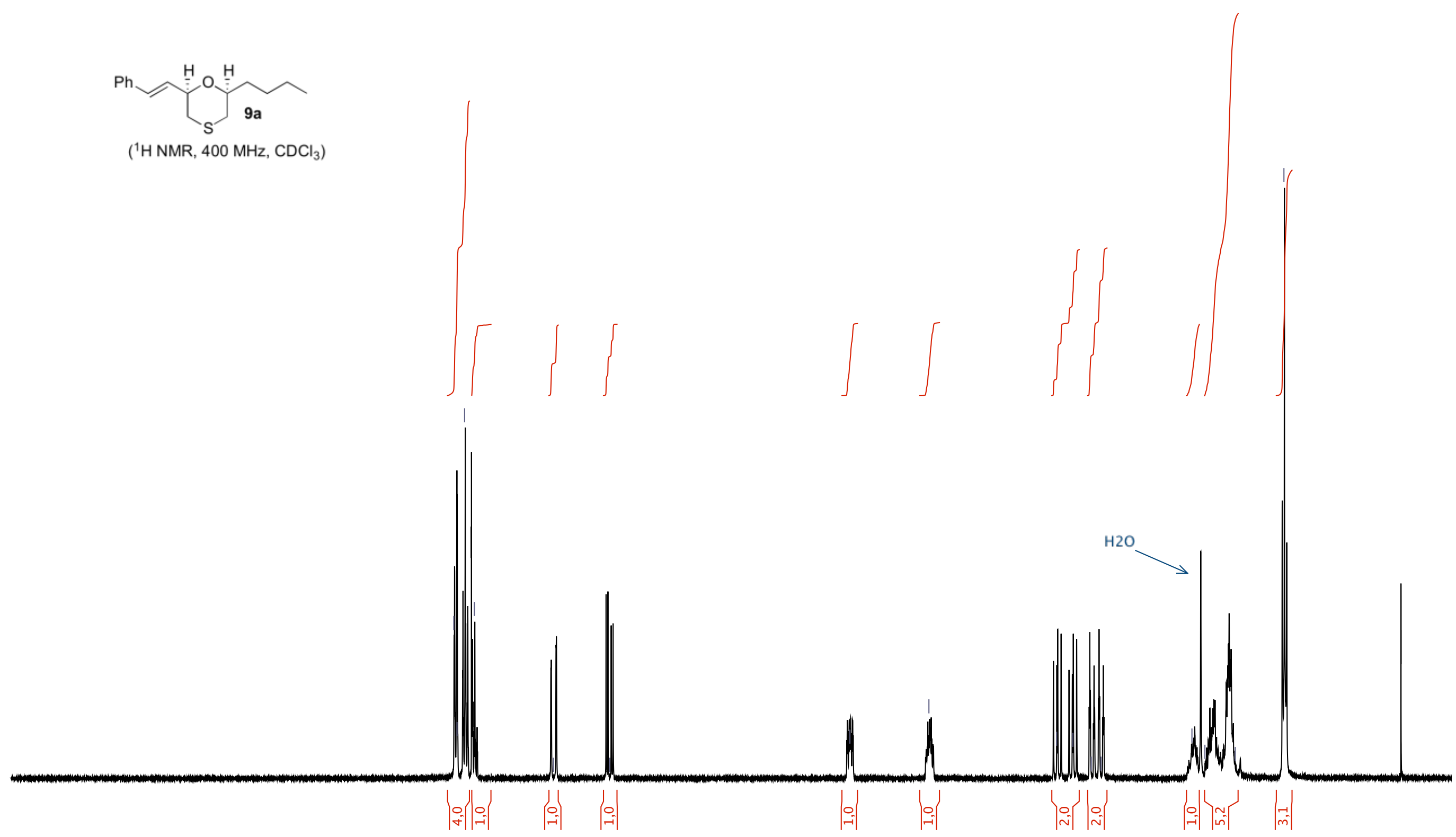


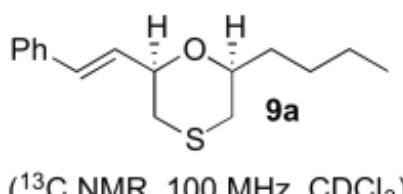

$\left({ }^{13} \mathrm{C}\right.$ NMR, $\left.100 \mathrm{MHz}, \mathrm{CDCl}_{3}\right)$ 


$$
\mathrm{Ph}
$$$$
{ }_{9 b}^{O} C_{P h}
$$

( ${ }^{1} \mathrm{H} \mathrm{NMR}, 400 \mathrm{MHz}, \mathrm{CDCl}_{3}$ )

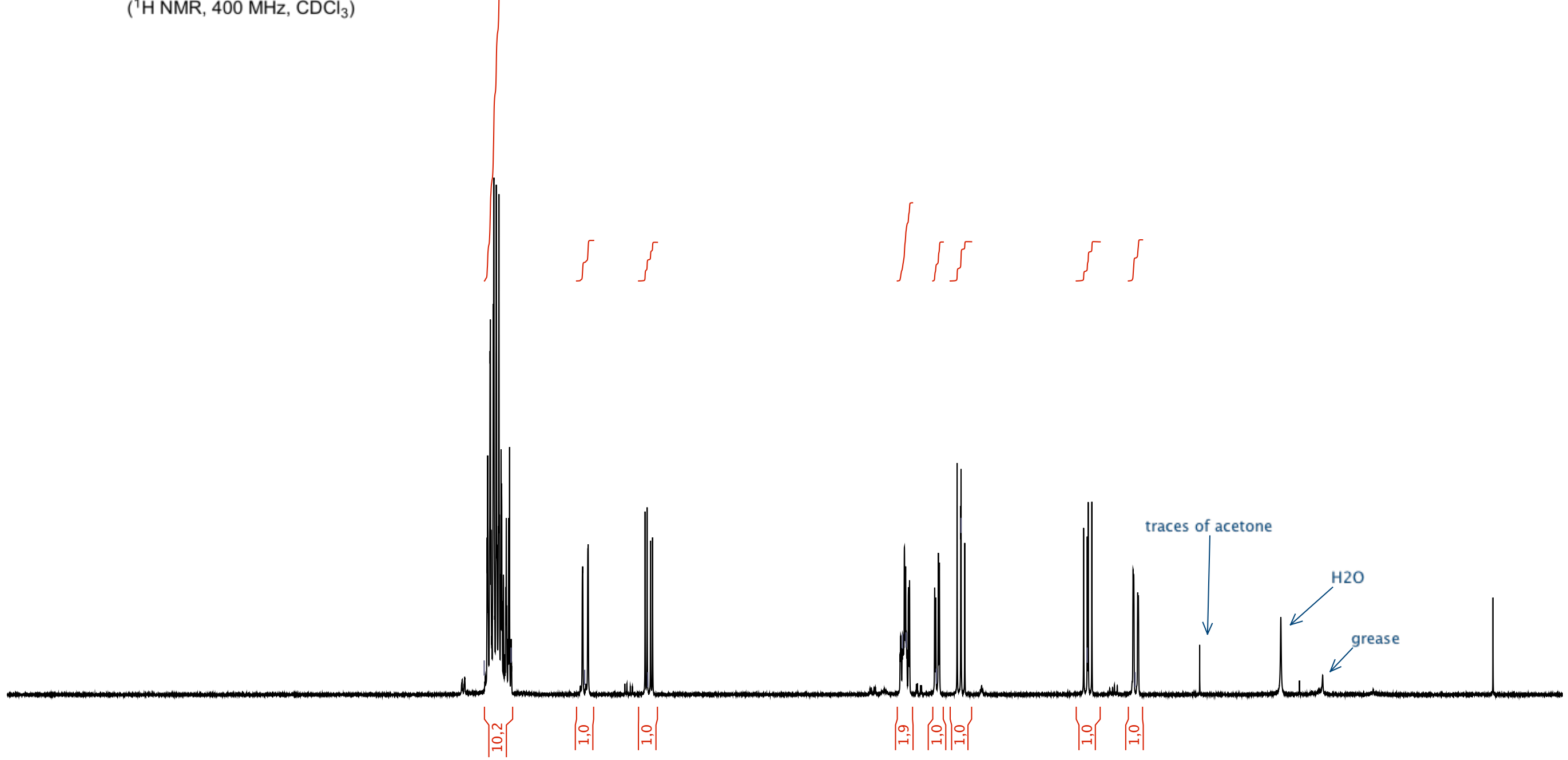


$\mathrm{Ph}$

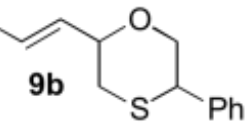

$\left({ }^{13} \mathrm{C}\right.$ NMR, $\left.100 \mathrm{MHz}, \mathrm{CDCl}_{3}\right)$

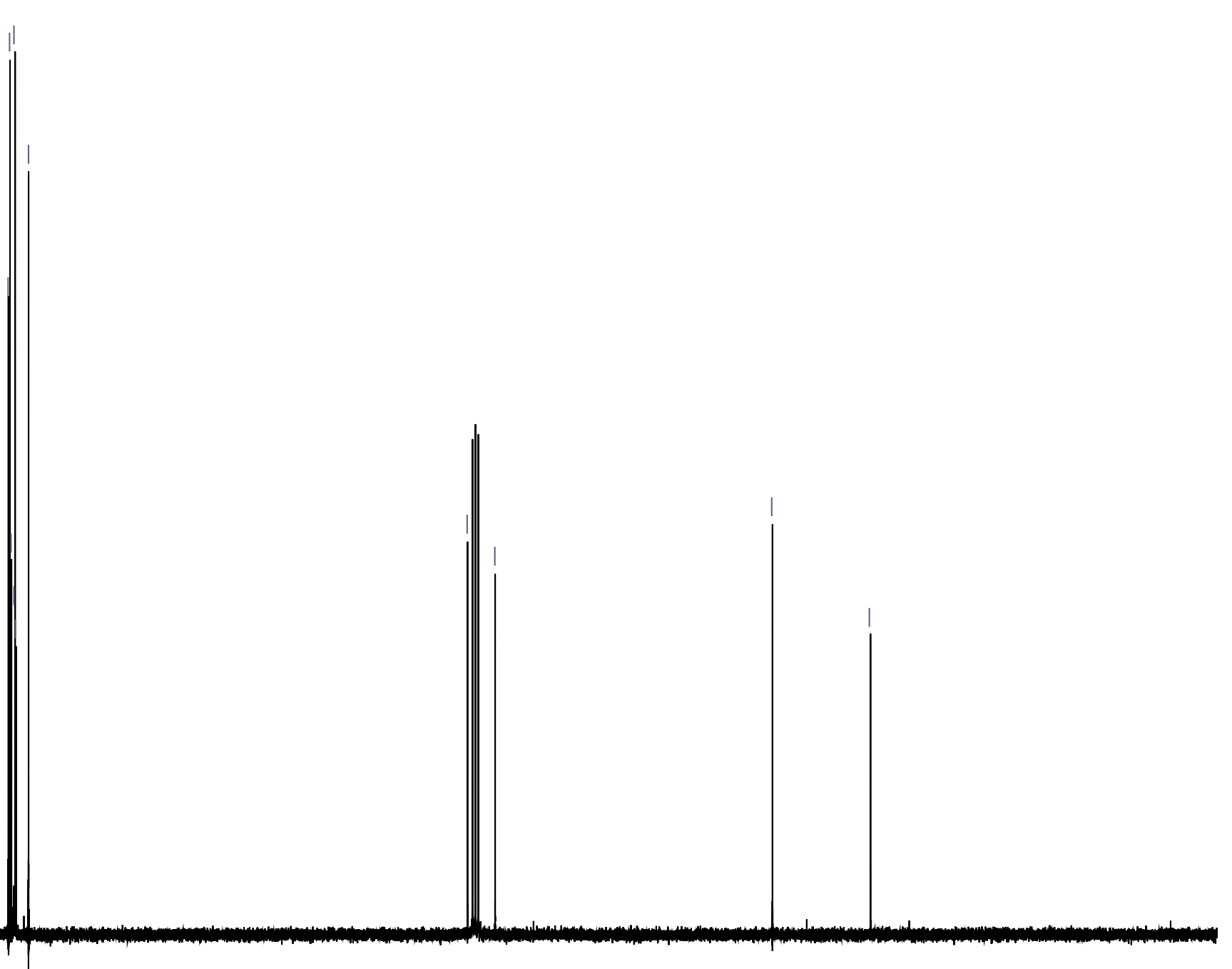


$$
\mathrm{Ph}
$$<smiles>CC1CC[C+]Sc2ccccc2C1</smiles>

$\left({ }^{1} \mathrm{H} \mathrm{NMR}, 400 \mathrm{MHz}, \mathrm{CDCl}_{3}\right.$ )
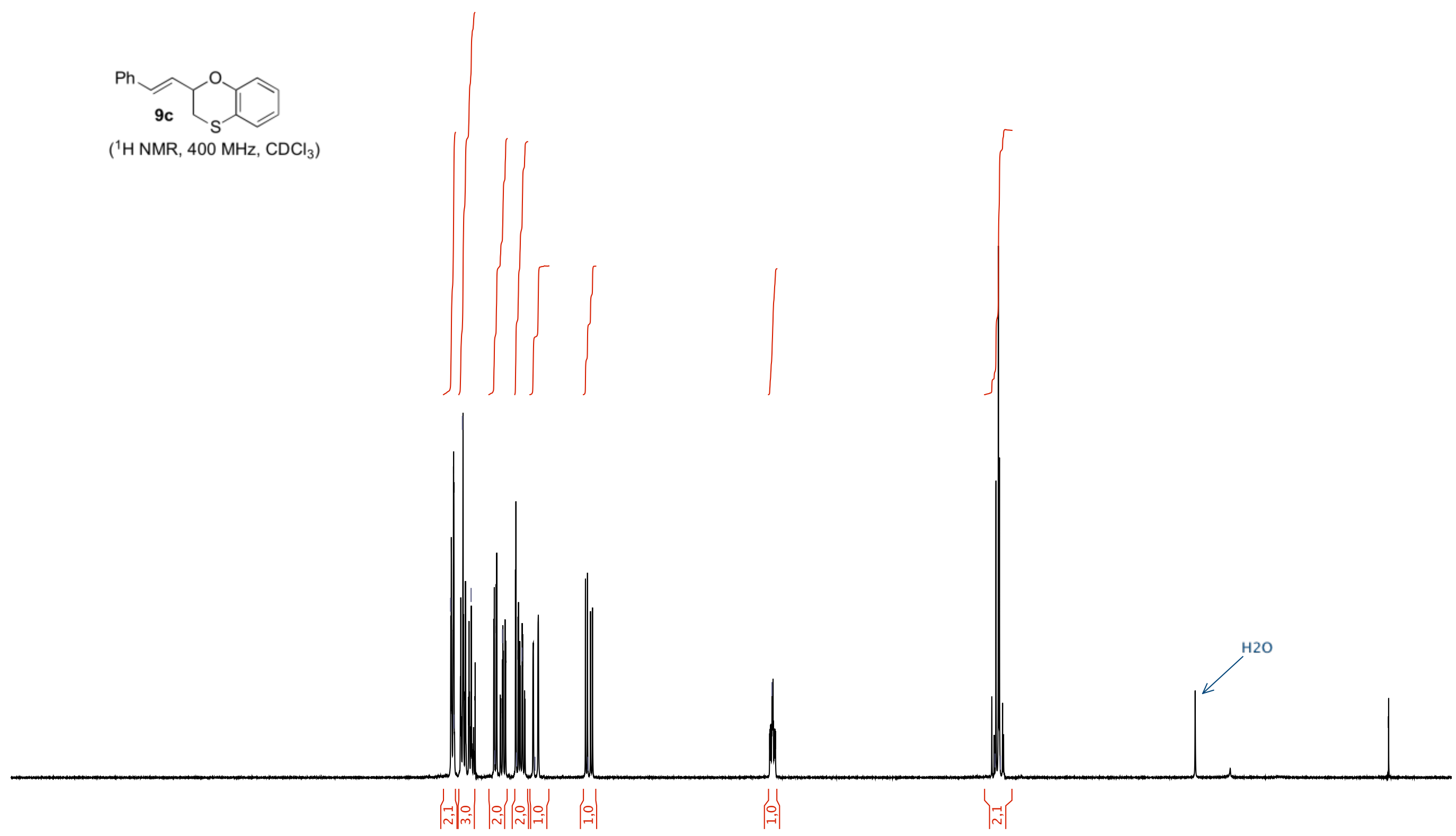
흘
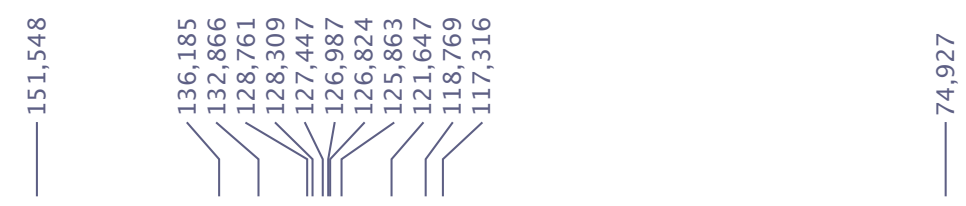

$\left({ }^{13} \mathrm{C} \mathrm{NMR}, 100 \mathrm{MHz}, \mathrm{CDCl}_{3}\right)$

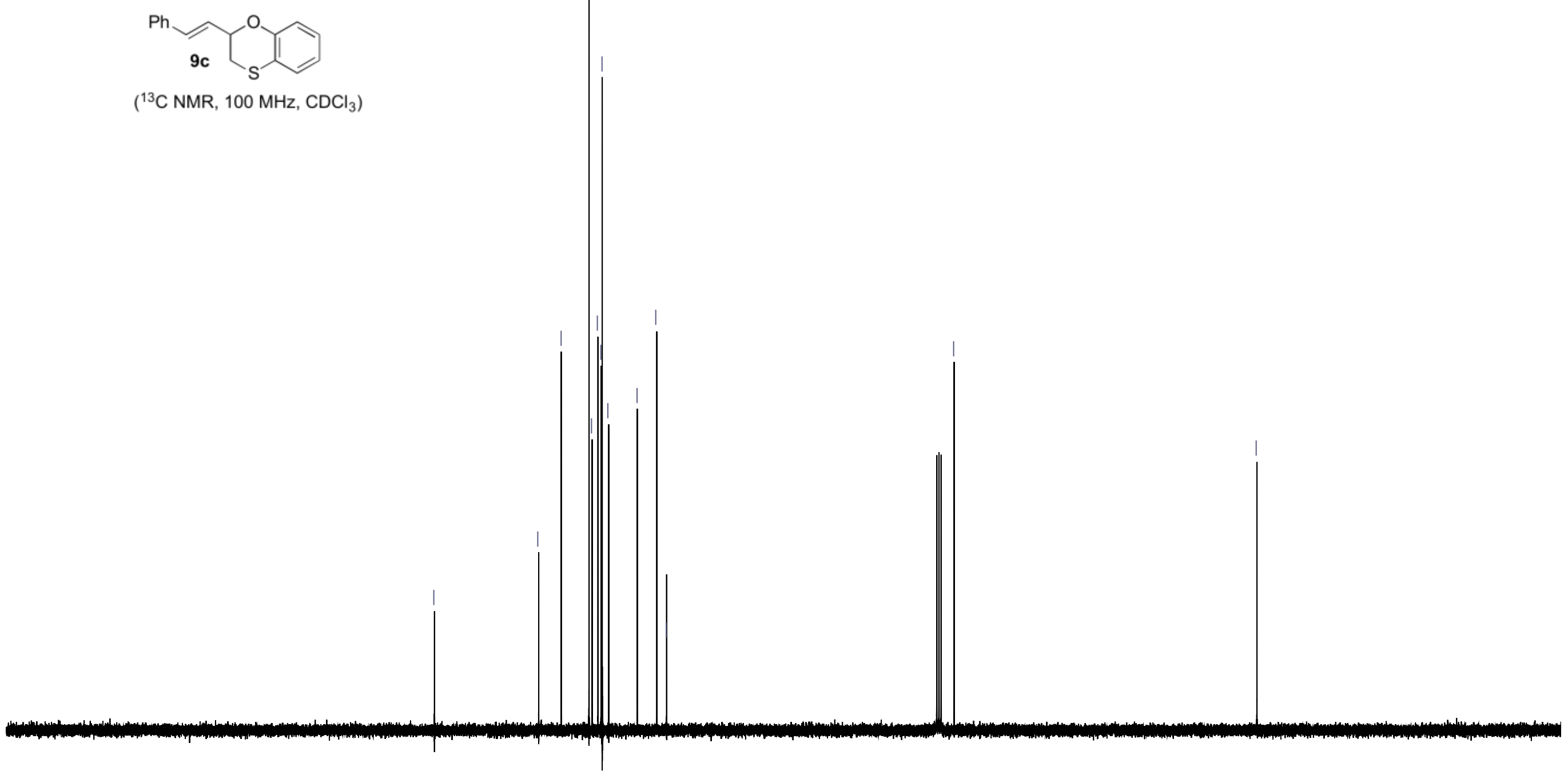

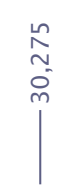

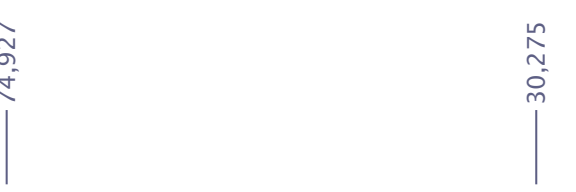

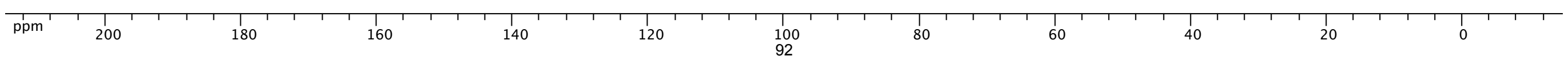




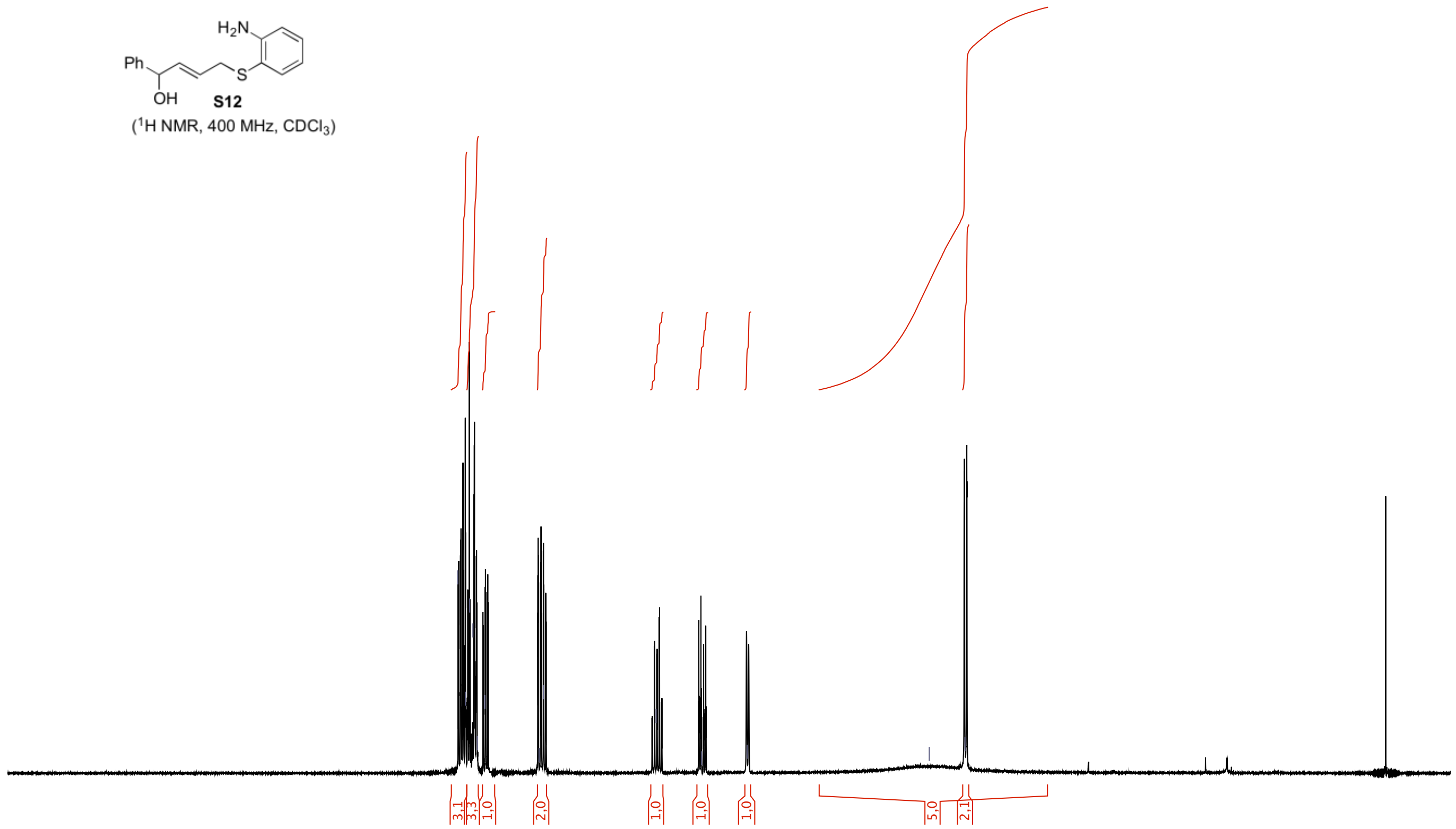




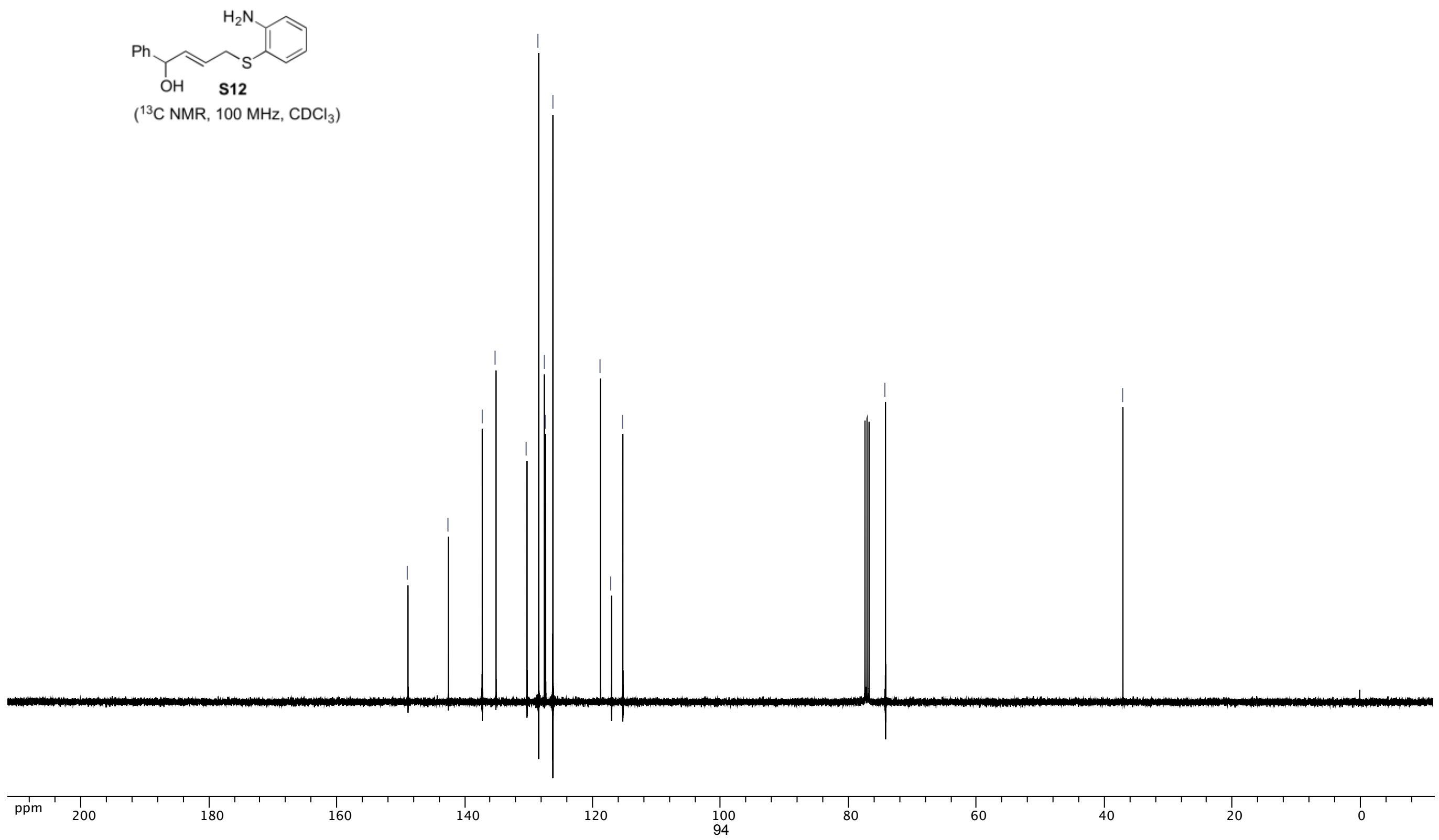




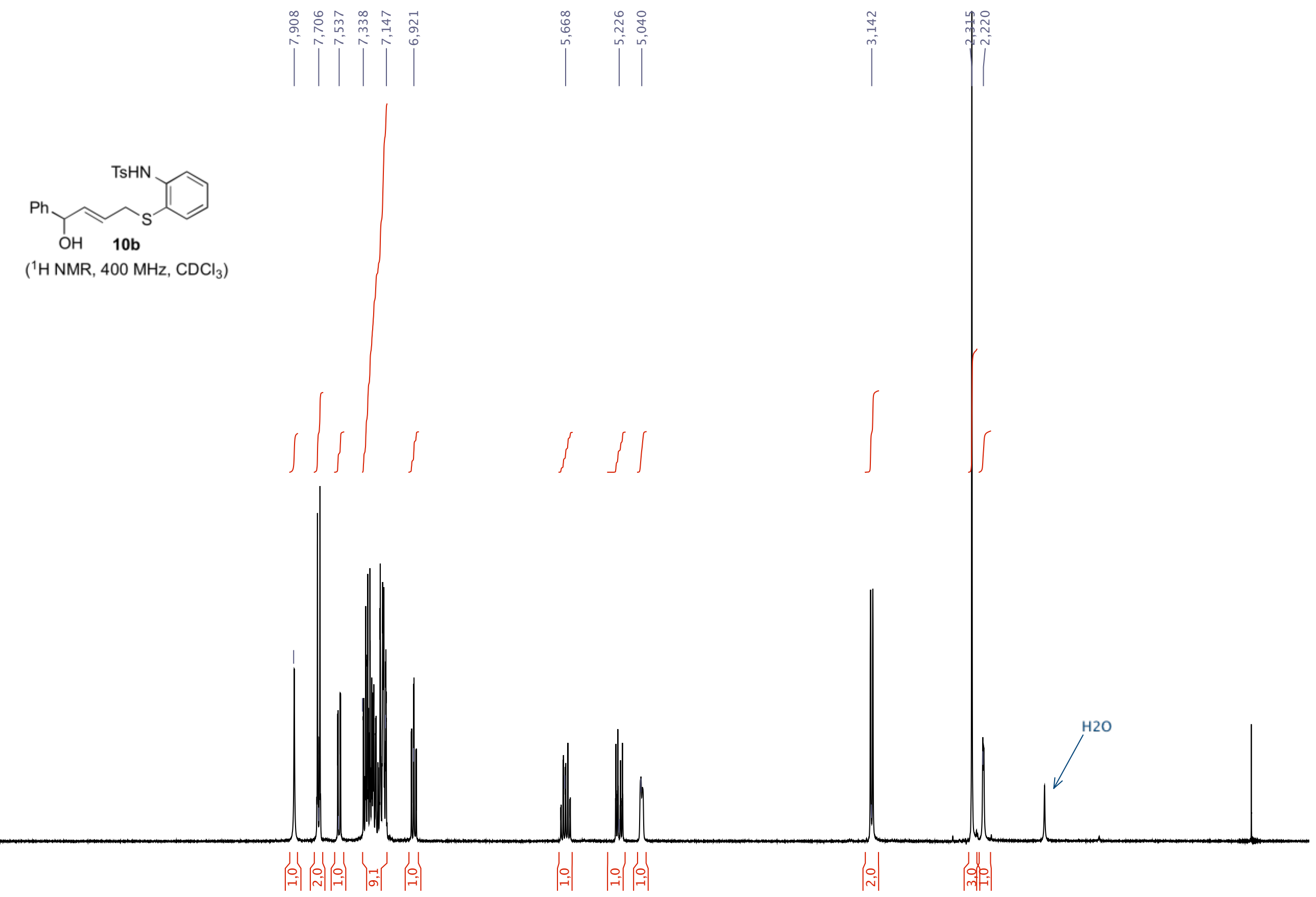




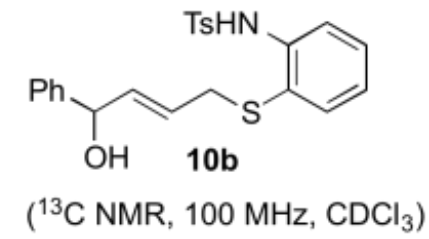

$\left({ }^{13} \mathrm{C}\right.$ NMR, $\left.100 \mathrm{MHz}, \mathrm{CDCl}_{3}\right)$ 
OTBS

$\mathrm{Pr}$

$\left({ }^{1} \mathrm{H} \mathrm{NMR}, 400 \mathrm{MHz}, \mathrm{CDCl}_{3}\right)$

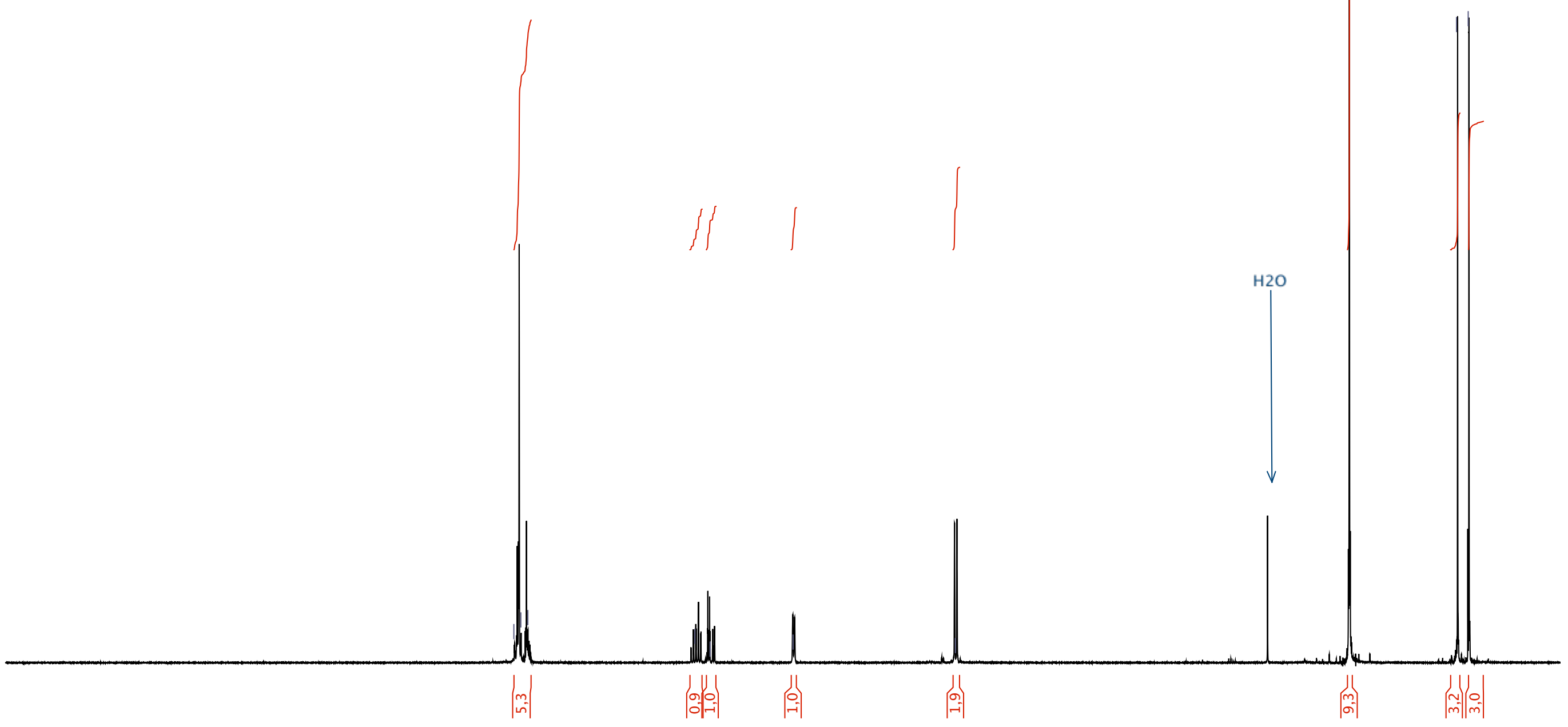




$$
\text { Br }
$$

$\left({ }^{13} \mathrm{C} \mathrm{NMR}, 100 \mathrm{MHz}, \mathrm{CDCl}_{3}\right)$

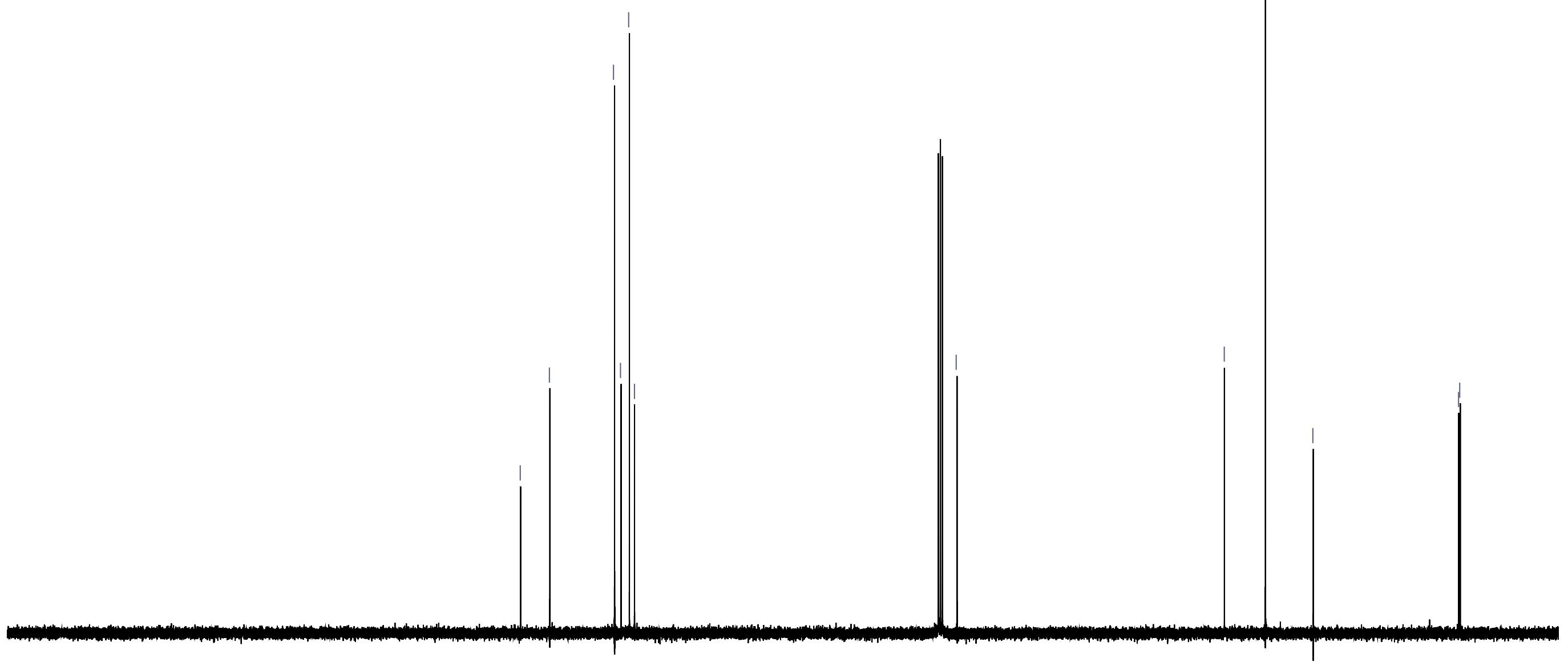




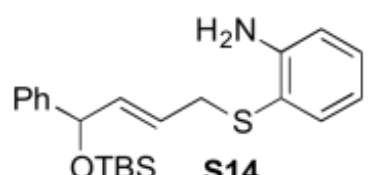

OTBS $\mathbf{5 1 4}$

( ${ }^{1} \mathrm{H}$ NMR, $400 \mathrm{MHz}, \mathrm{CDCl}_{3}$ )
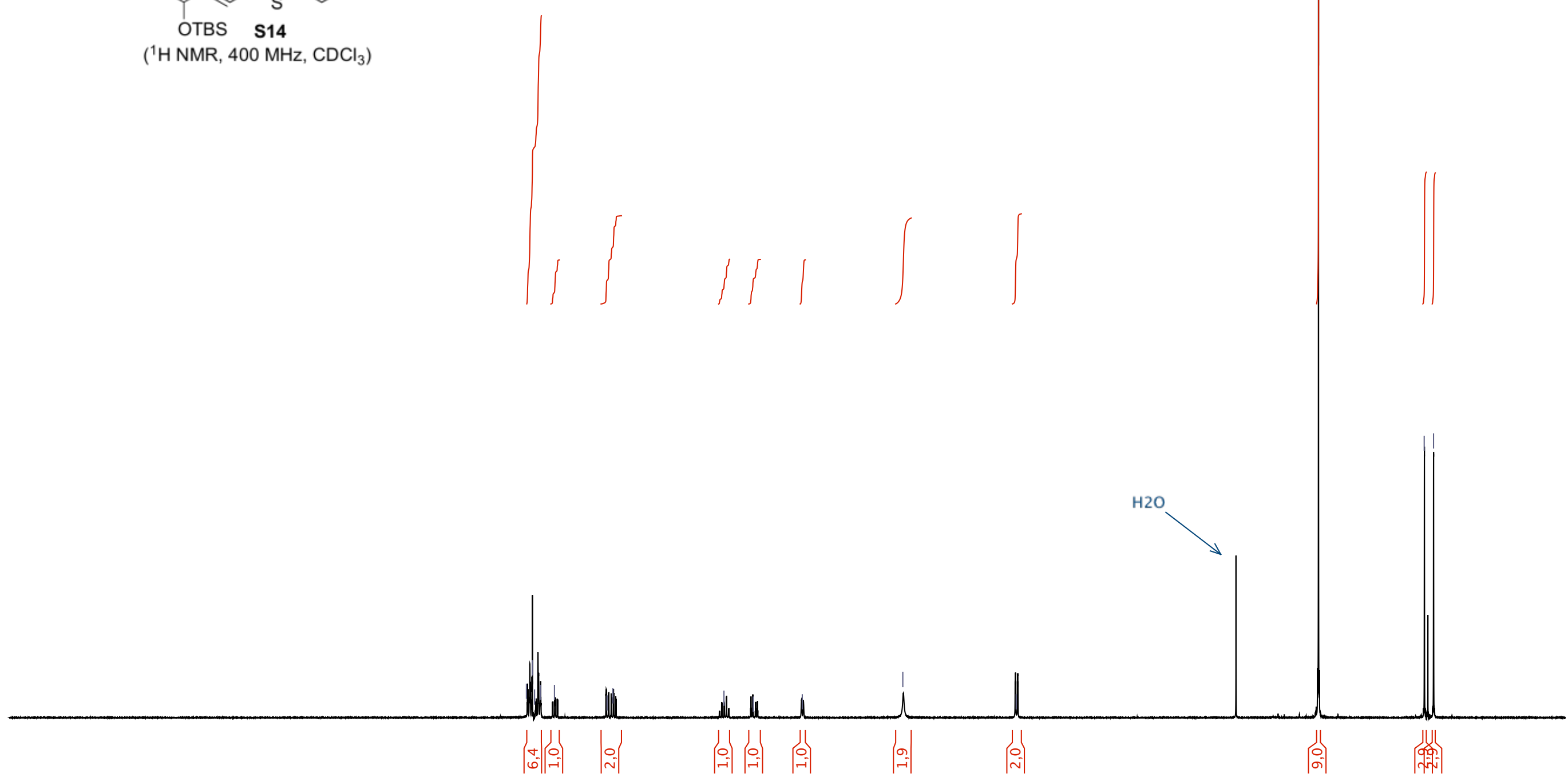


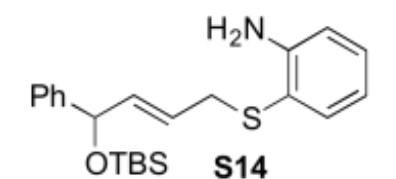

${ }^{3} \mathrm{C}$ NMR, $100 \mathrm{MHz}, \mathrm{CDCl}_{3}$ ) 

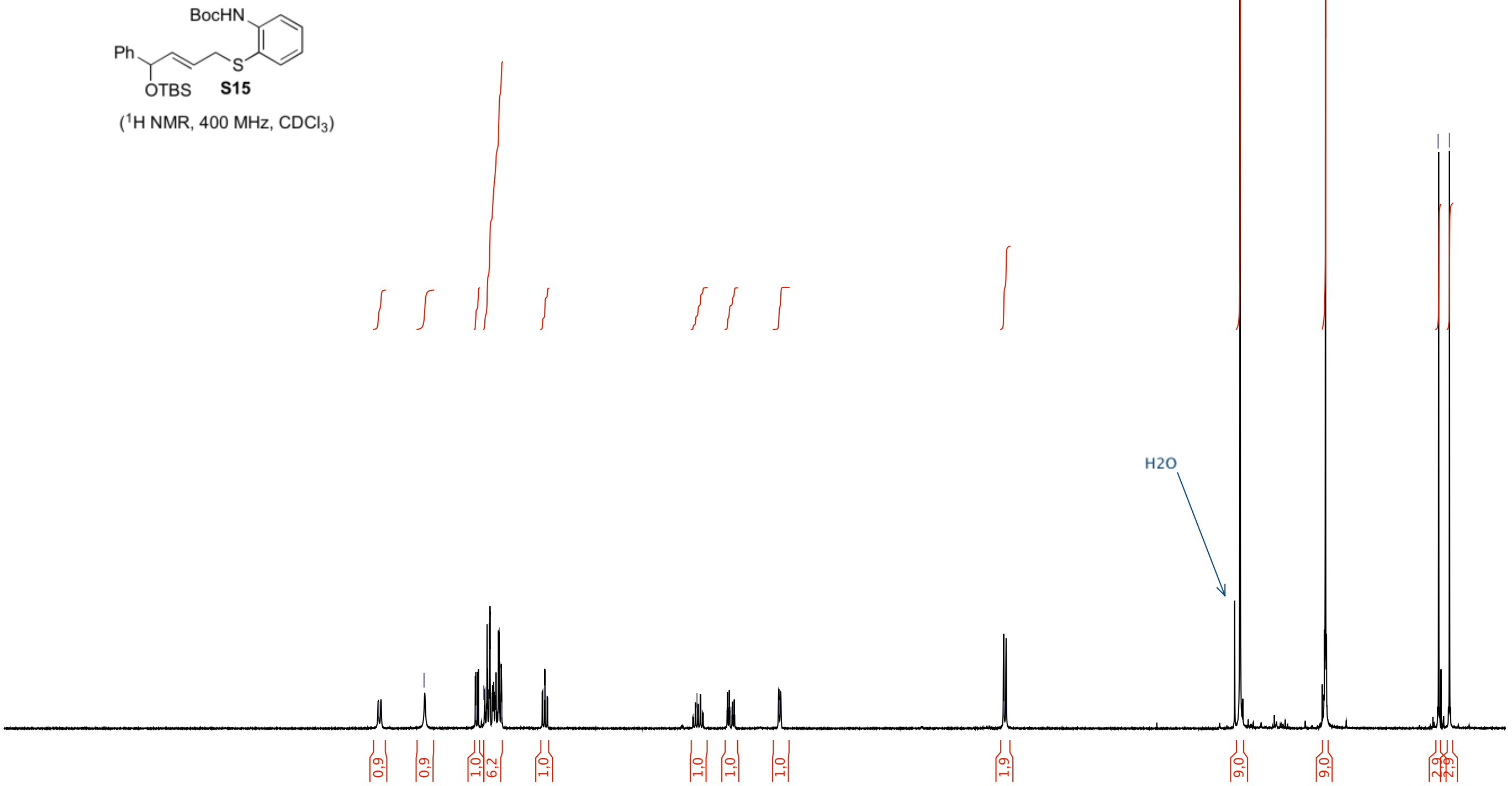


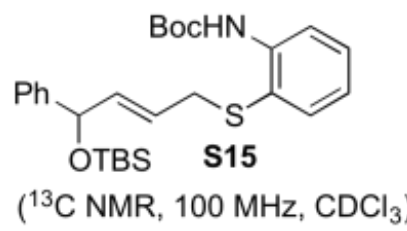

$\left({ }^{13} \mathrm{C} \mathrm{NMR}, 100 \mathrm{MHz}, \mathrm{CDCl}_{3}\right)$ 


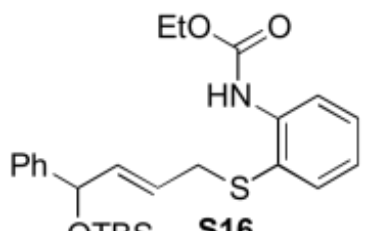

OTBS $\mathbf{S 1 6}$

( ${ }^{1} \mathrm{H}$ NMR, $400 \mathrm{MHz}, \mathrm{CDCl}_{3}$ )
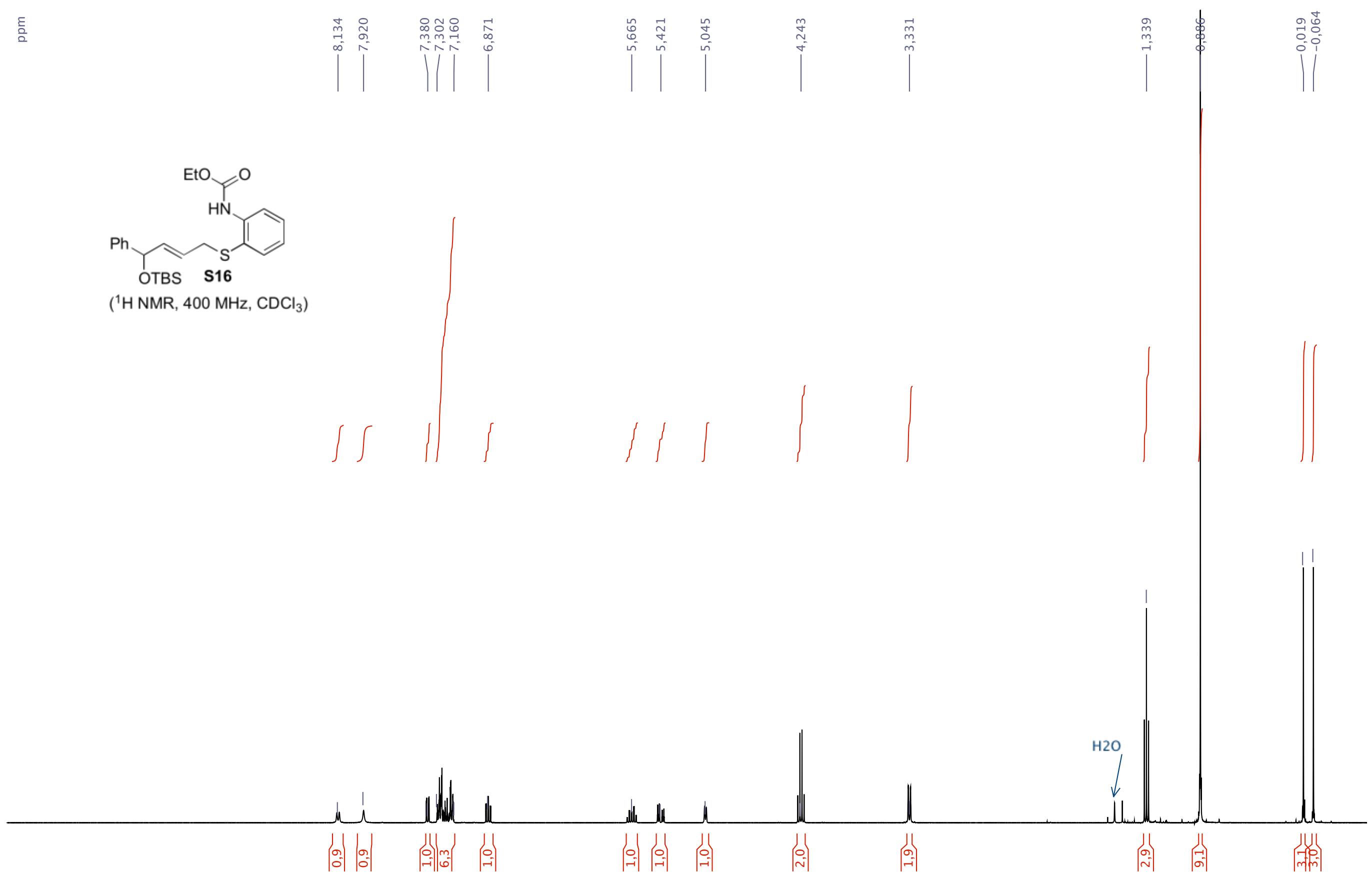


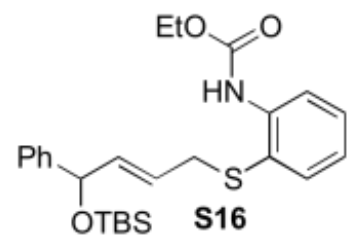

$\left({ }^{13} \mathrm{C} \mathrm{NMR}, 100 \mathrm{MHz}, \mathrm{CDCl}_{3}\right)$ 


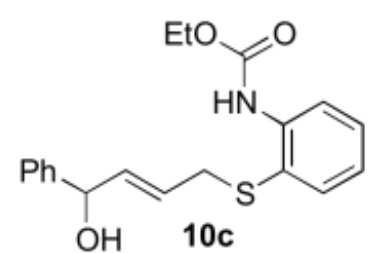

$\left({ }^{1} \mathrm{H} \mathrm{NMR}, 400 \mathrm{MHz}, \mathrm{CDCl}_{3}\right.$ )
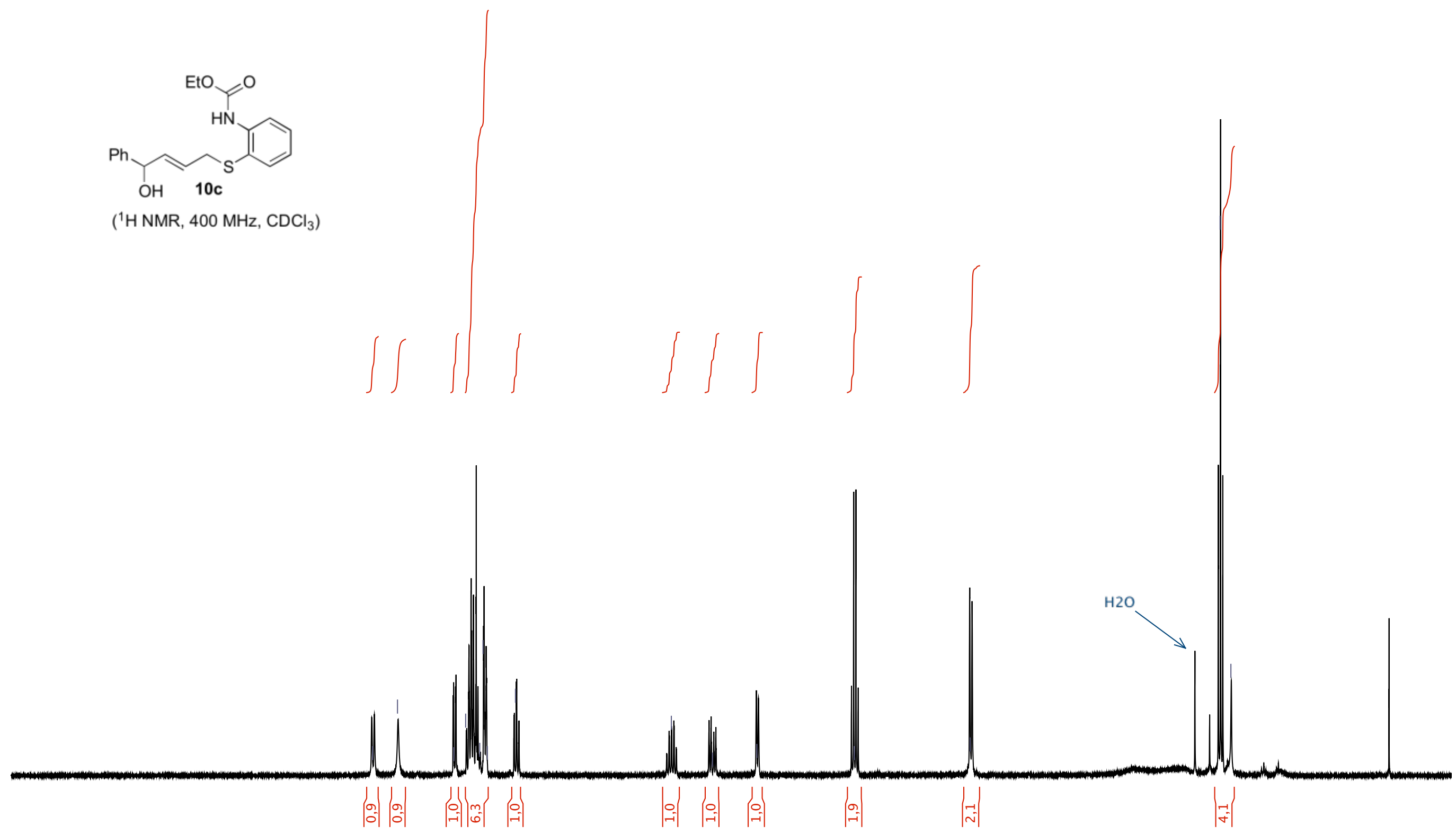
흠
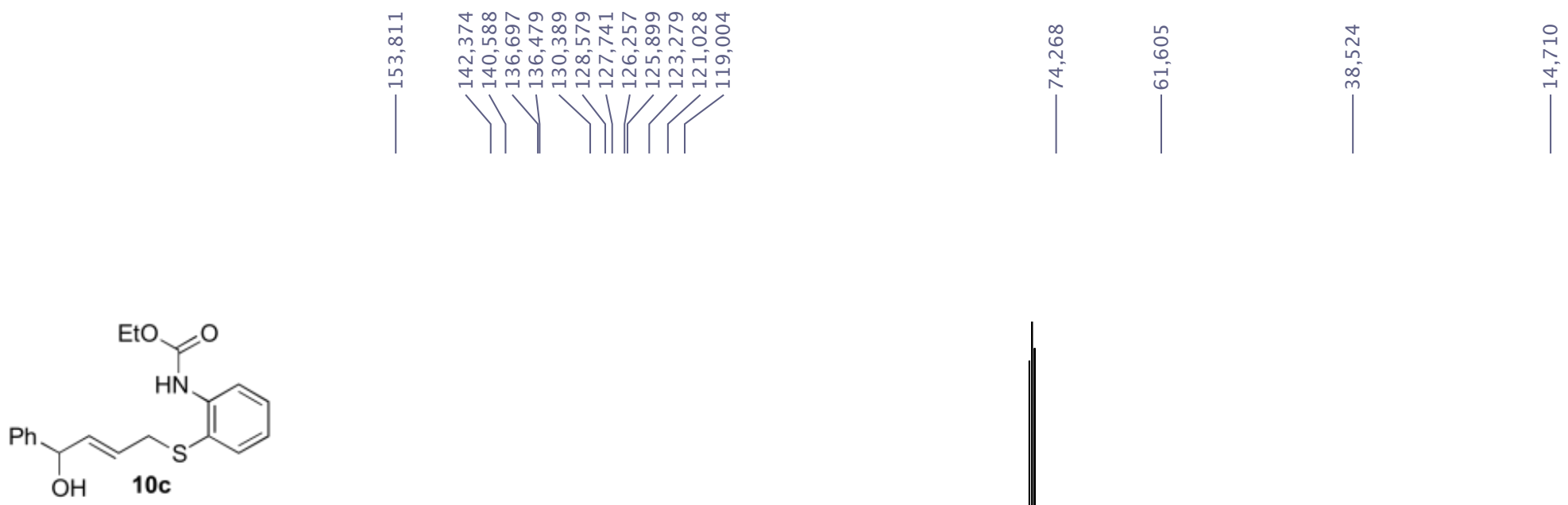

$\left({ }^{13} \mathrm{C} \mathrm{NMR}, 100 \mathrm{MHz}, \mathrm{CDCl}_{3}\right)$

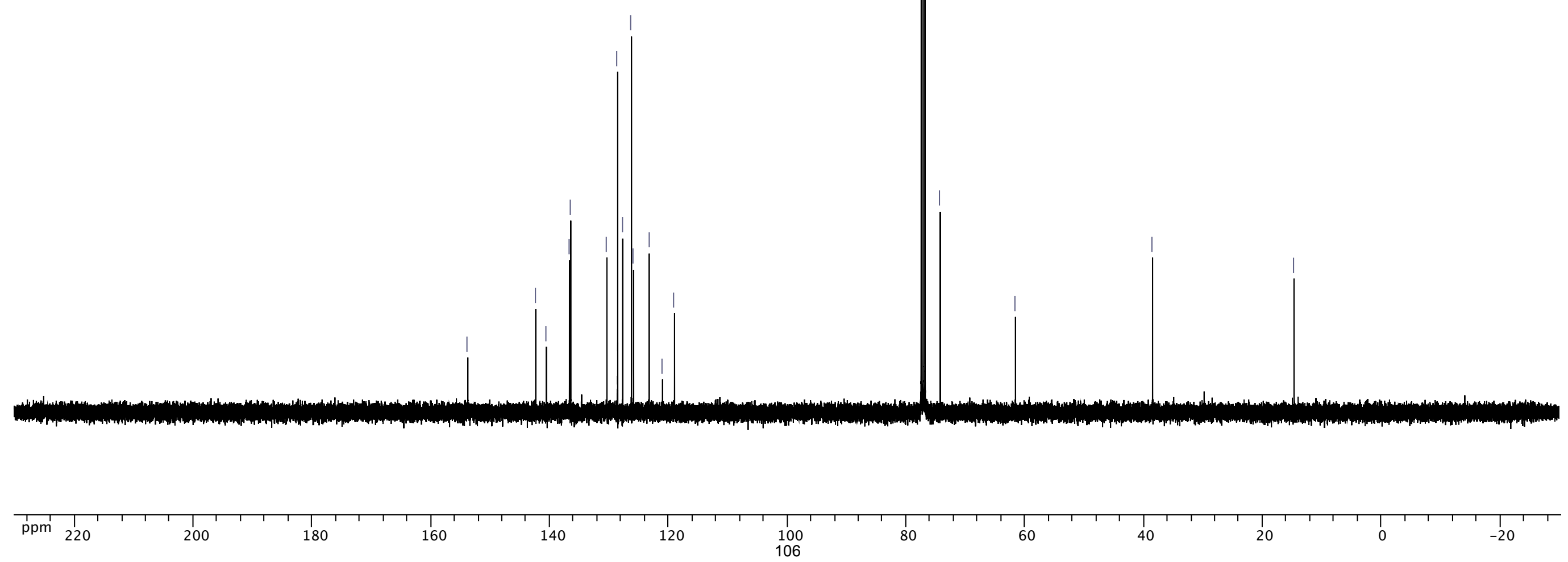




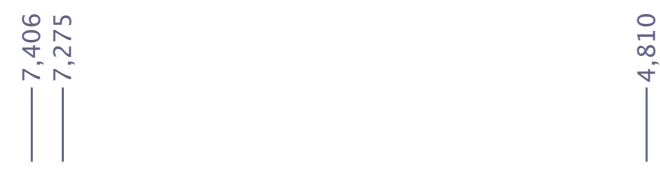

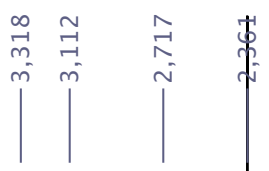

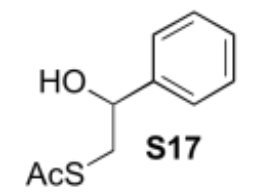

$\left({ }^{1} \mathrm{H} \mathrm{NMR}, 400 \mathrm{MHz}, \mathrm{CDCl}_{3}\right)$
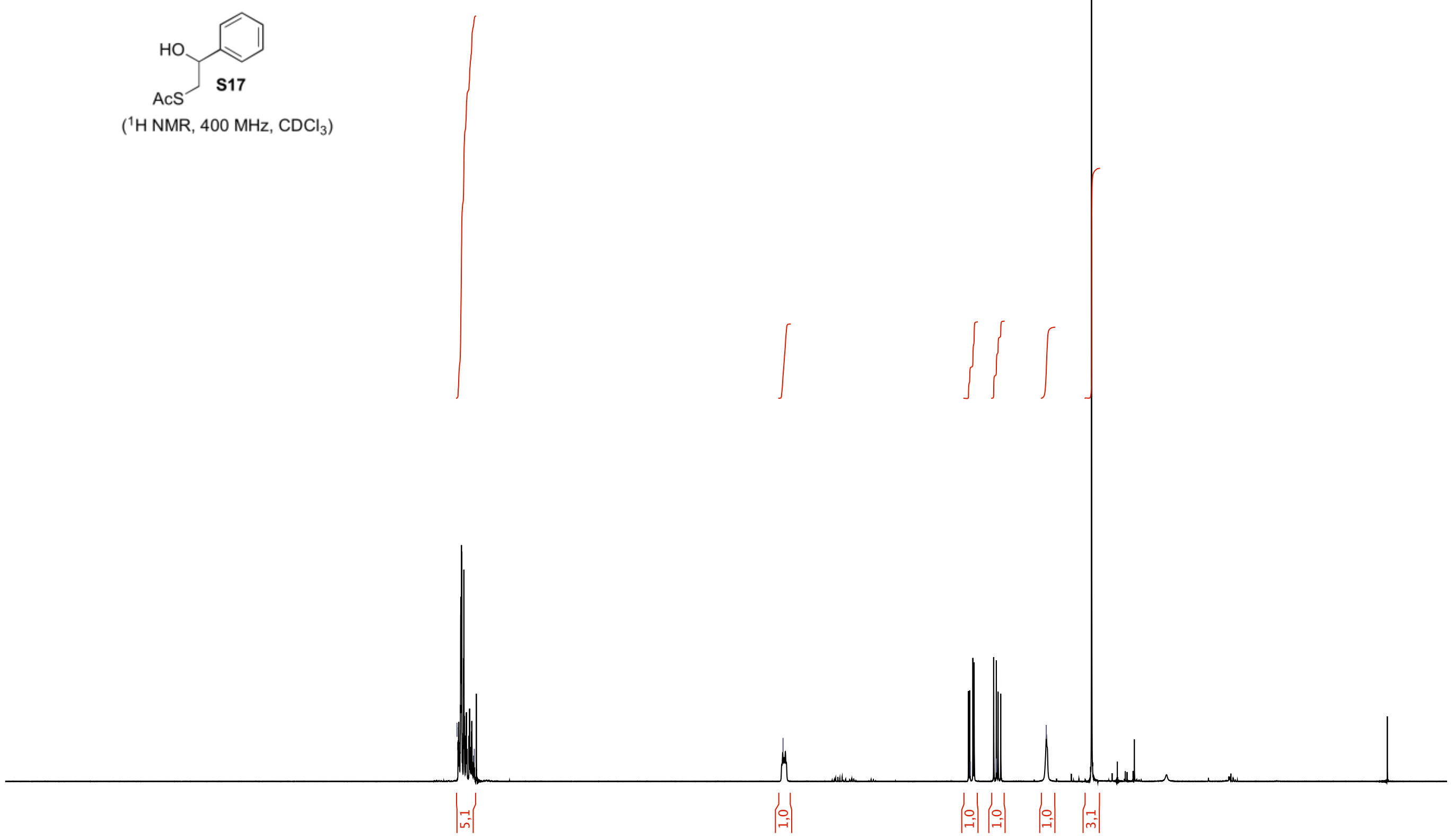


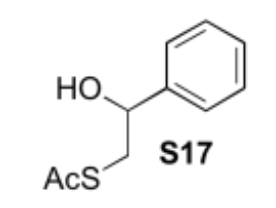

$\left({ }^{13} \mathrm{C} \mathrm{NMR}, 100 \mathrm{MHz}, \mathrm{CDCl}_{3}\right)$

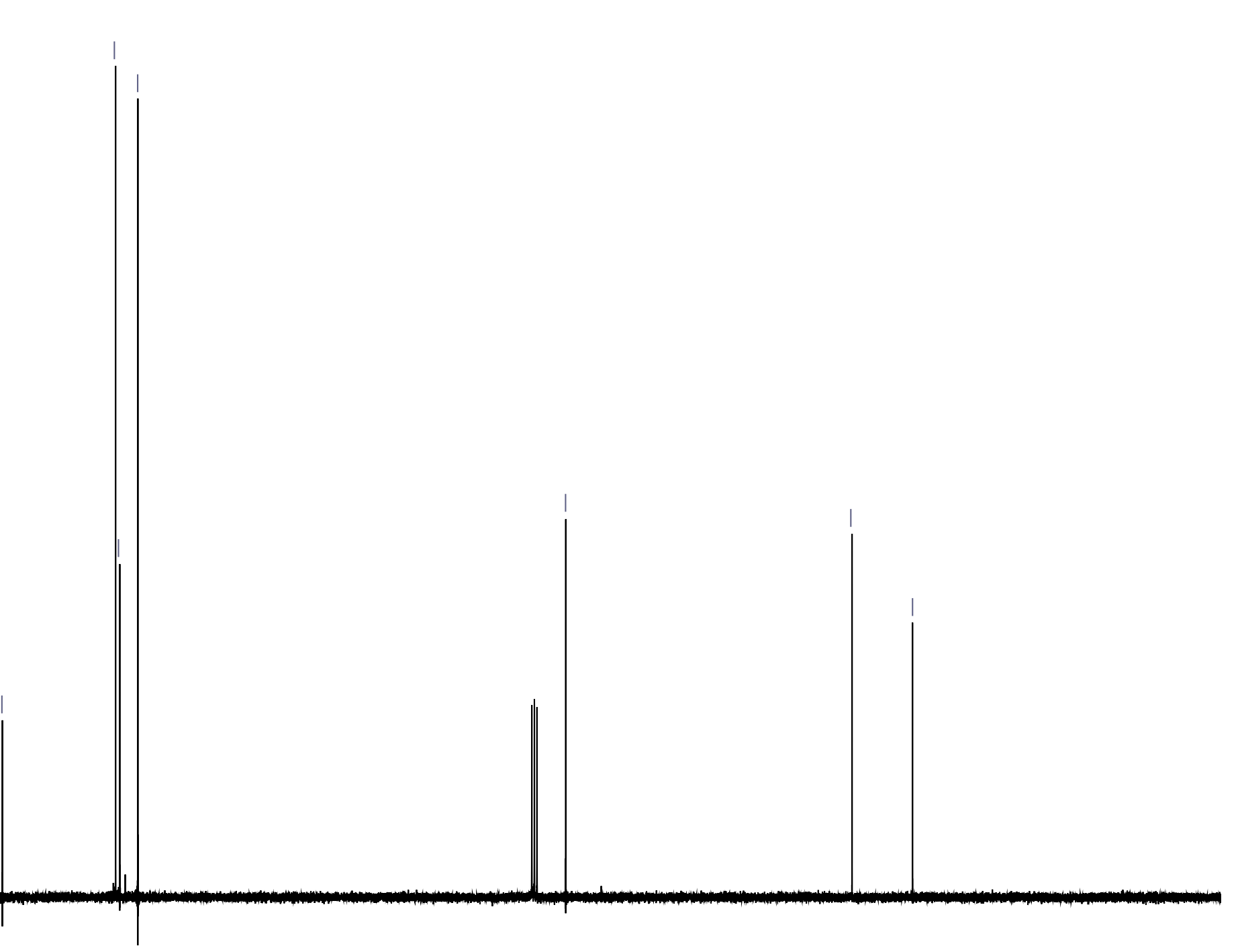



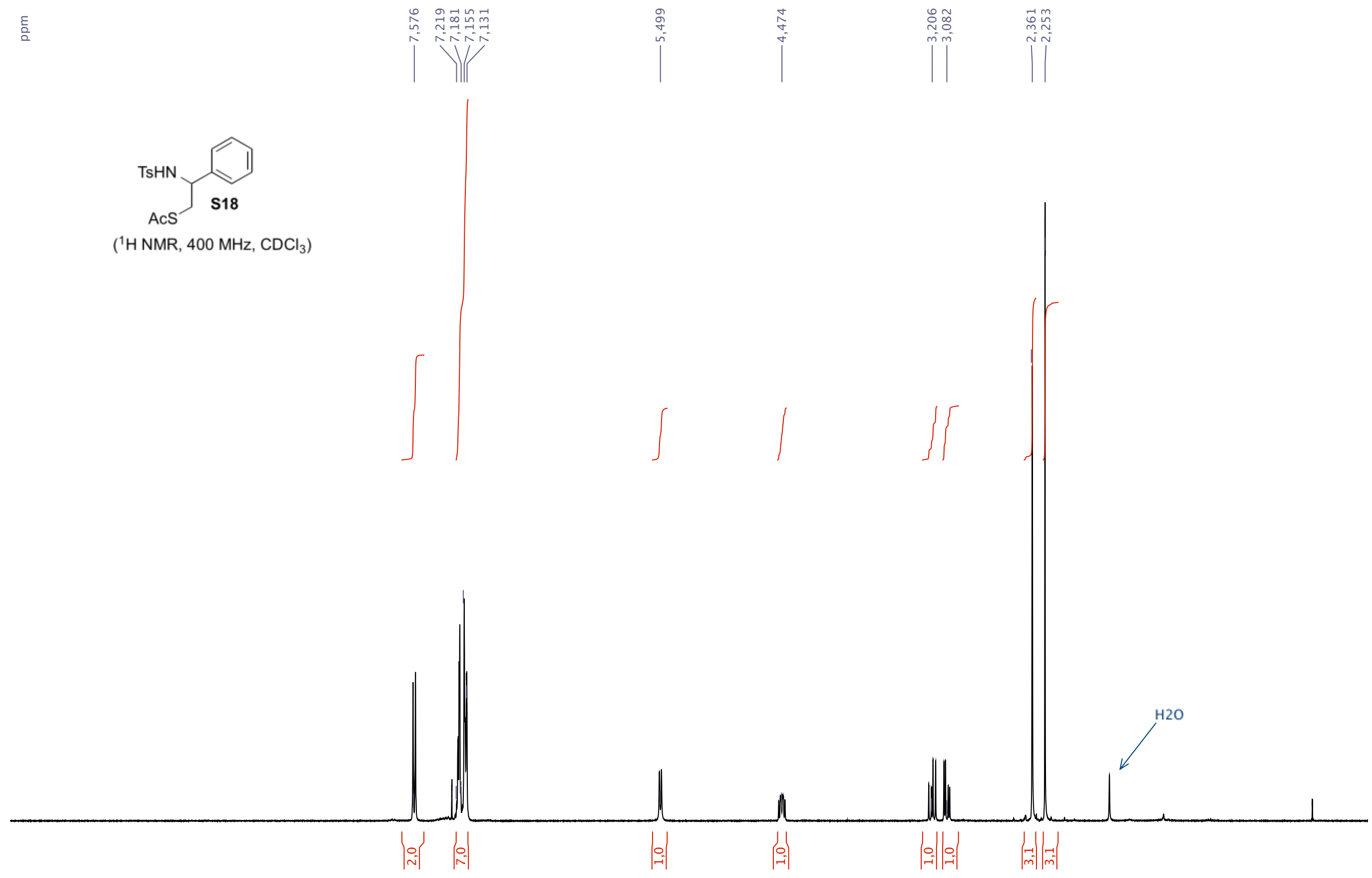


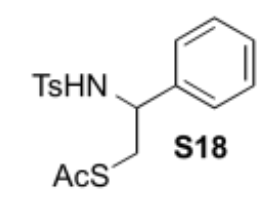

$\left({ }^{13} \mathrm{C} \mathrm{NMR}, 100 \mathrm{MHz}, \mathrm{CDCl}_{3}\right)$

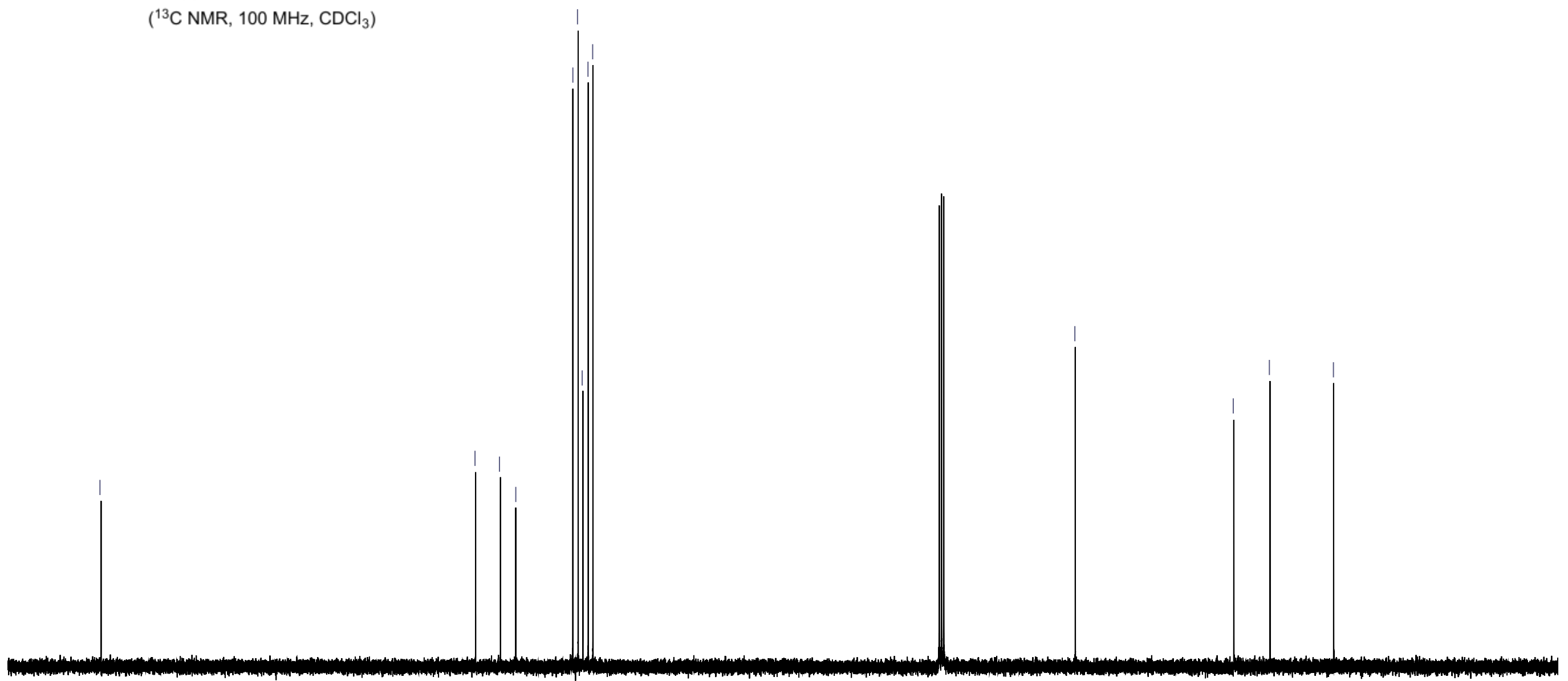




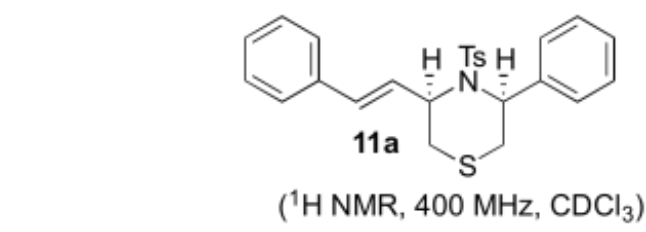

( ${ }^{1} \mathrm{H} \mathrm{NMR}, 400 \mathrm{MHz}, \mathrm{CDCl}_{3}$ )

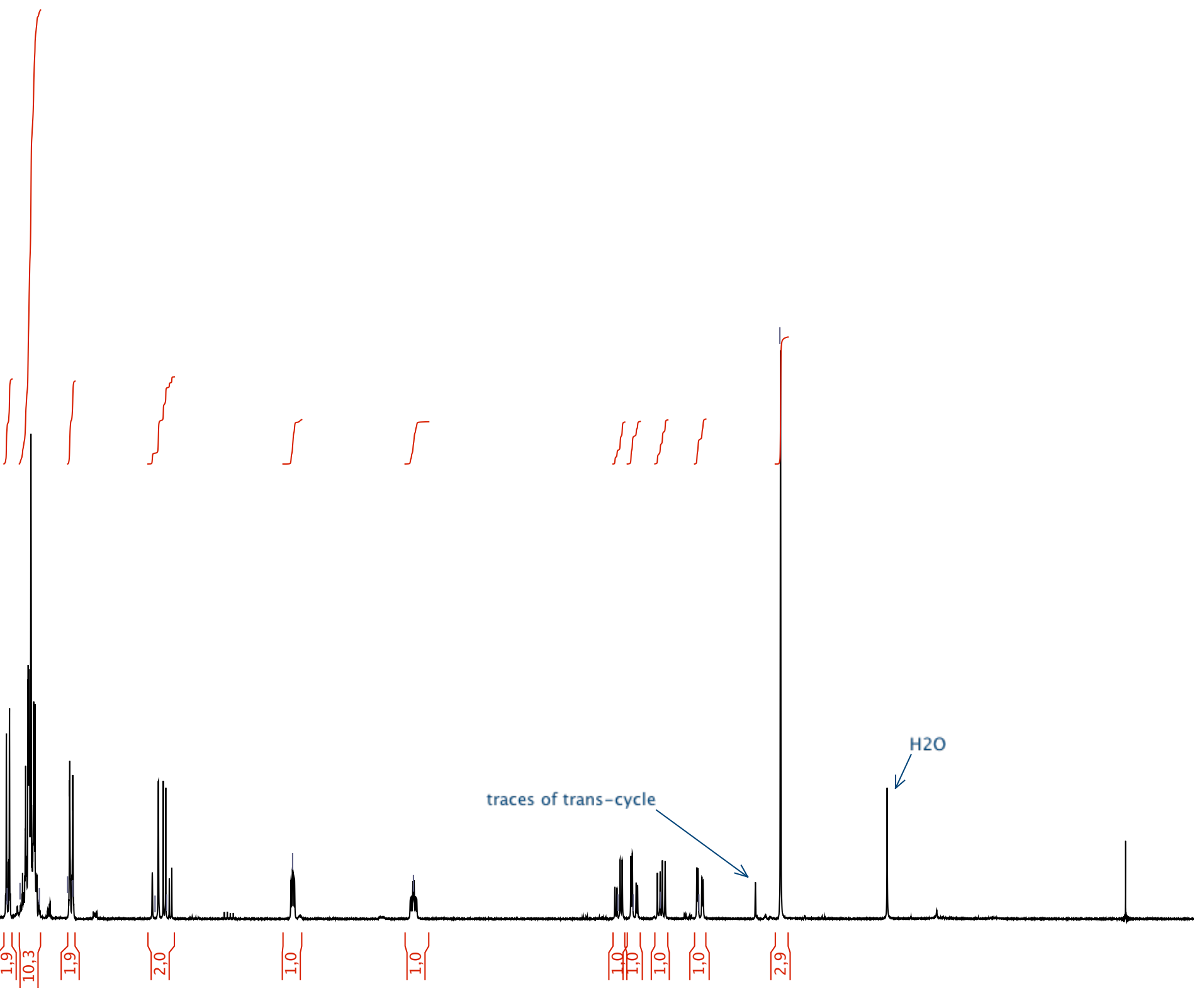




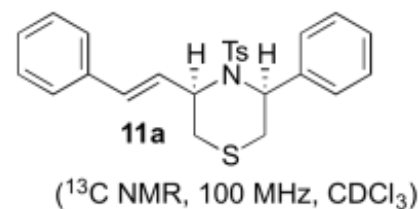

$\left({ }^{13} \mathrm{C} \mathrm{NMR}, 100 \mathrm{MHz}, \mathrm{CDCl}_{3}\right)$

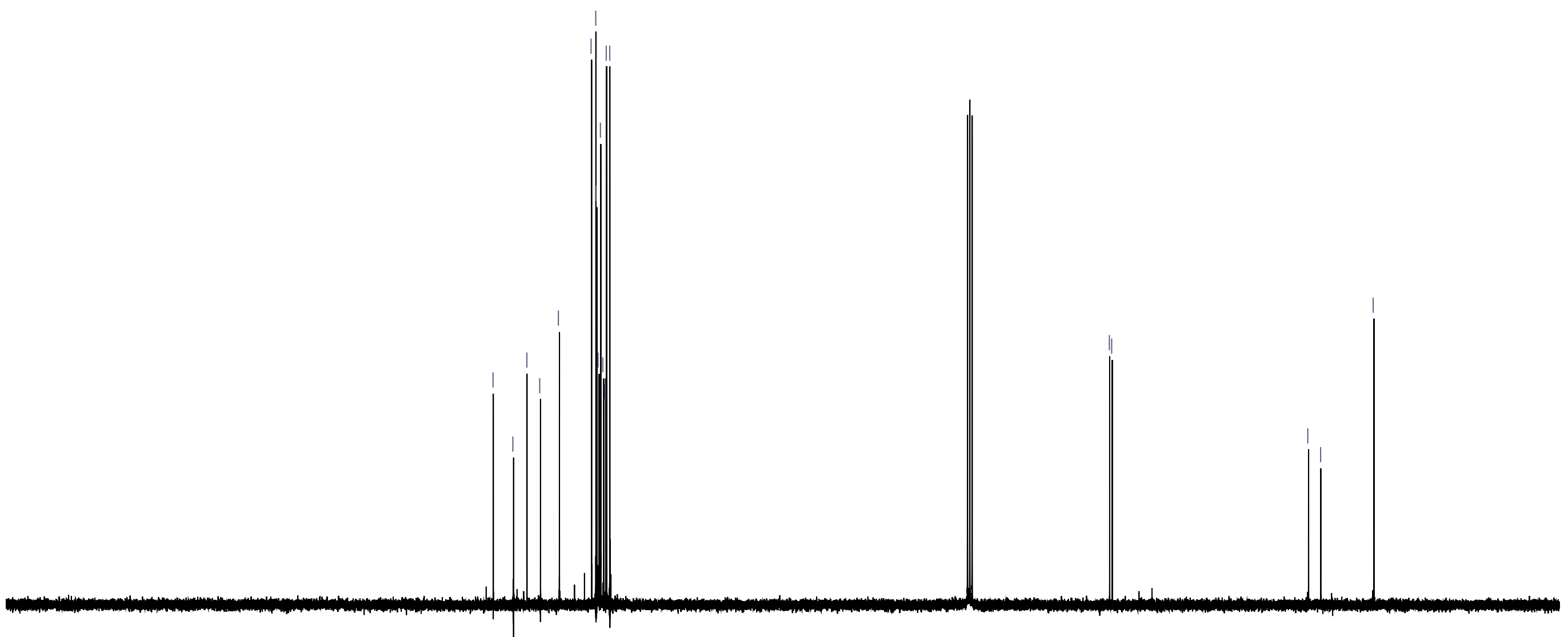




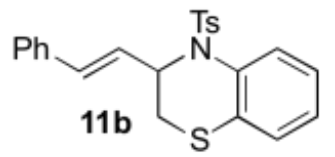

$\left({ }^{1} \mathrm{H} \mathrm{NMR}, 400 \mathrm{MHz}, \mathrm{CDCl}_{3}\right)$

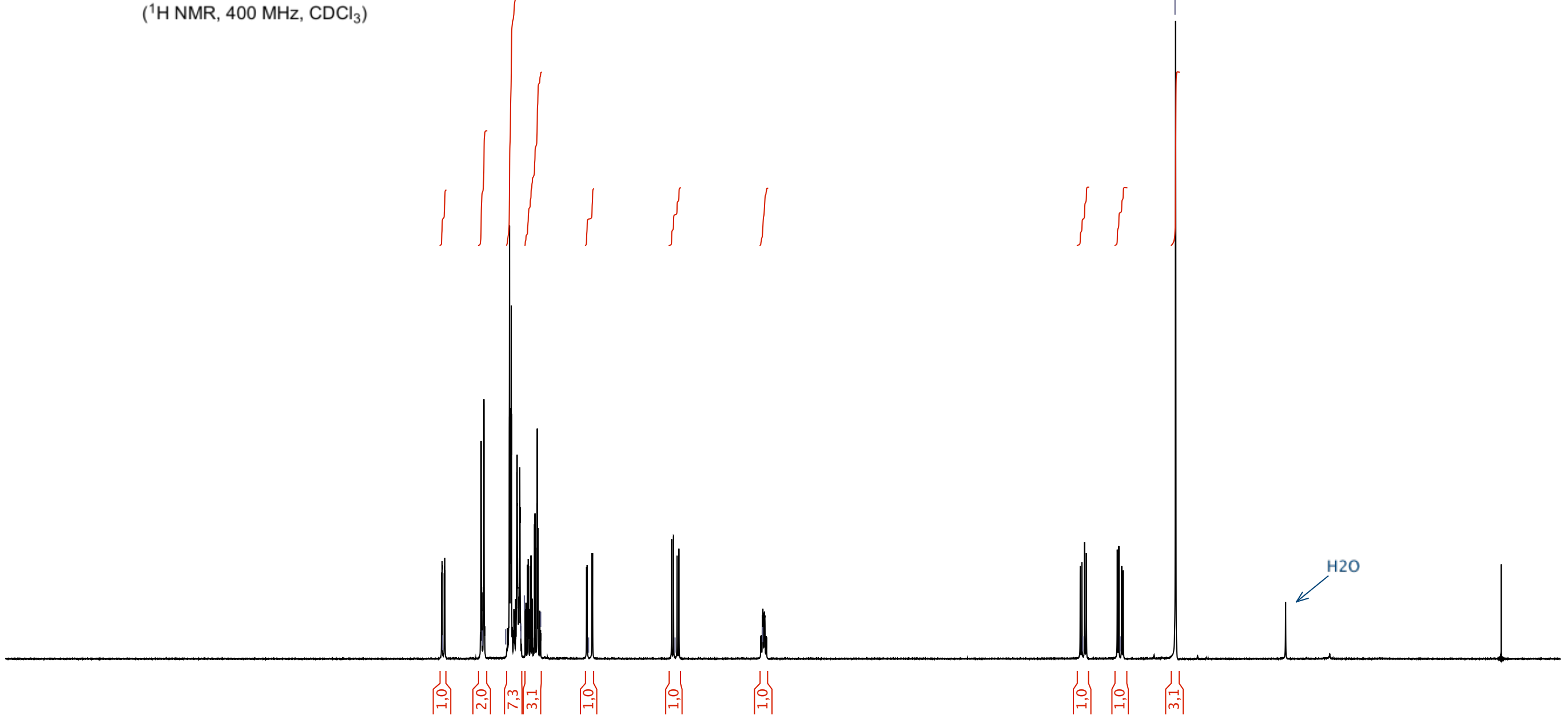




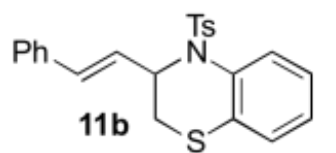

$\left({ }^{13} \mathrm{C}\right.$ NMR, $\left.100 \mathrm{MHz}, \mathrm{CDCl}_{3}\right)$

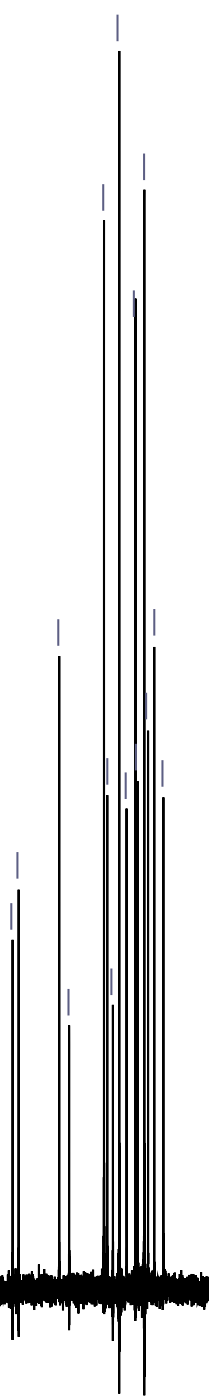




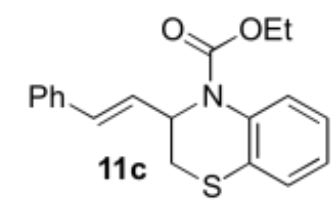

( ${ }^{1} \mathrm{H}$ NMR, $400 \mathrm{MHz}, \mathrm{CDCl}_{3}$ )

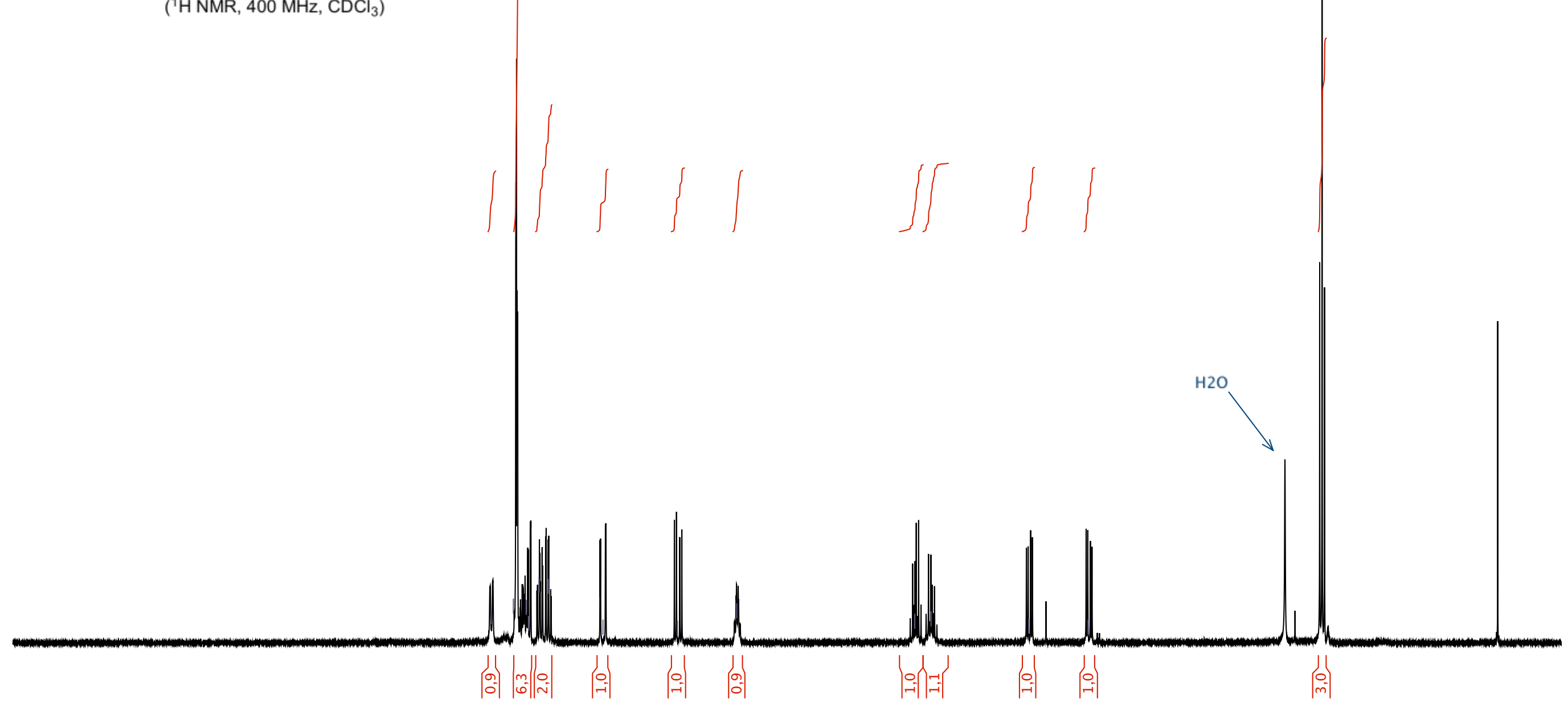


틈
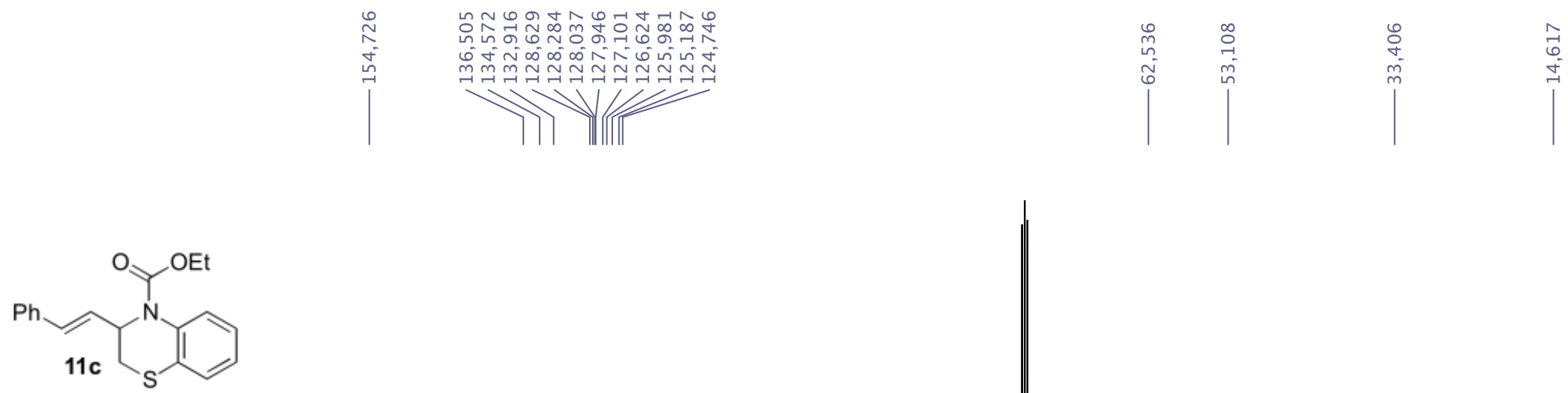

$\left({ }^{13} \mathrm{C} \mathrm{NMR}, 100 \mathrm{MHz}, \mathrm{CDCl}_{3}\right)$
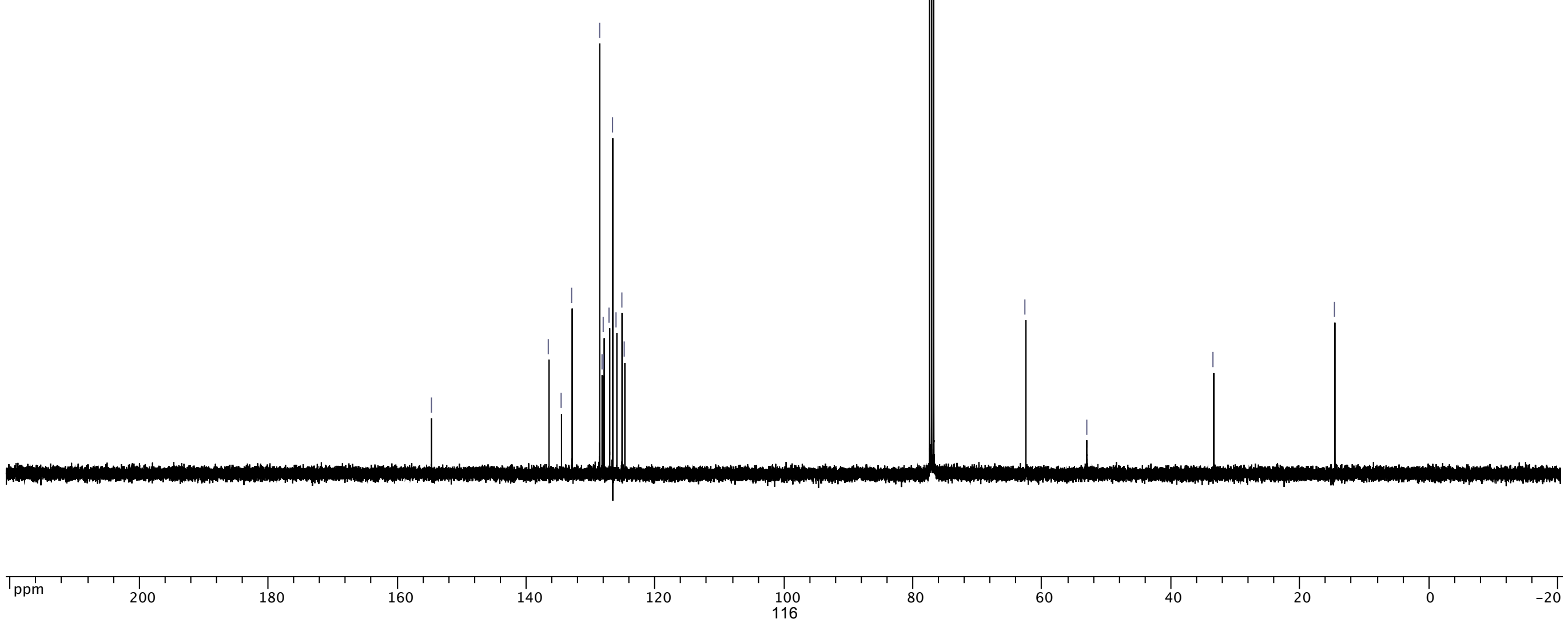


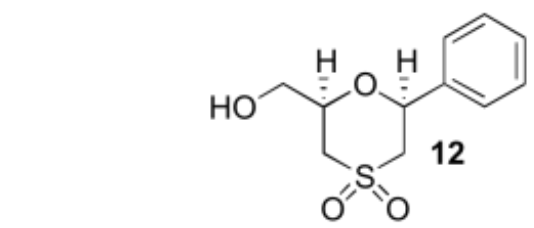

$\left({ }^{1} \mathrm{H} \mathrm{NMR}, 400 \mathrm{MHz}, \mathrm{CDCl}_{3}\right.$ )

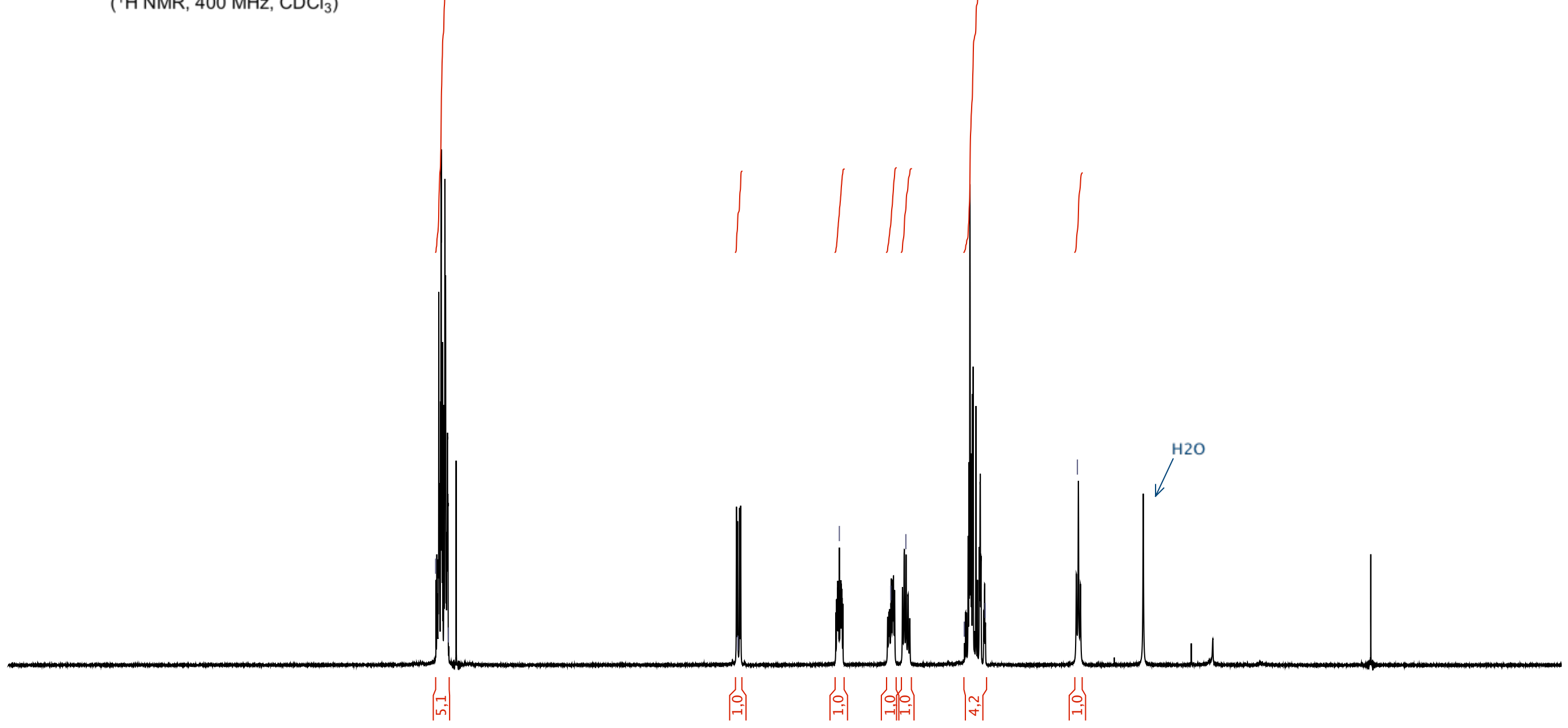




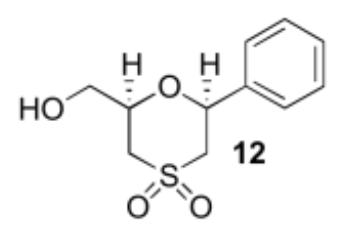

$\left({ }^{13} \mathrm{C} \mathrm{NMR}, 100 \mathrm{MHz}, \mathrm{CDCl}_{3}\right)$ 
틈

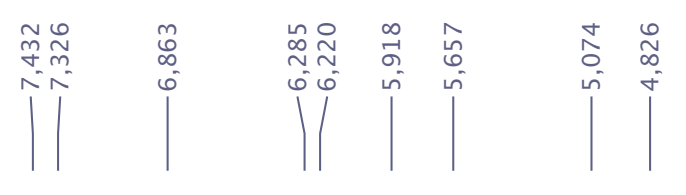
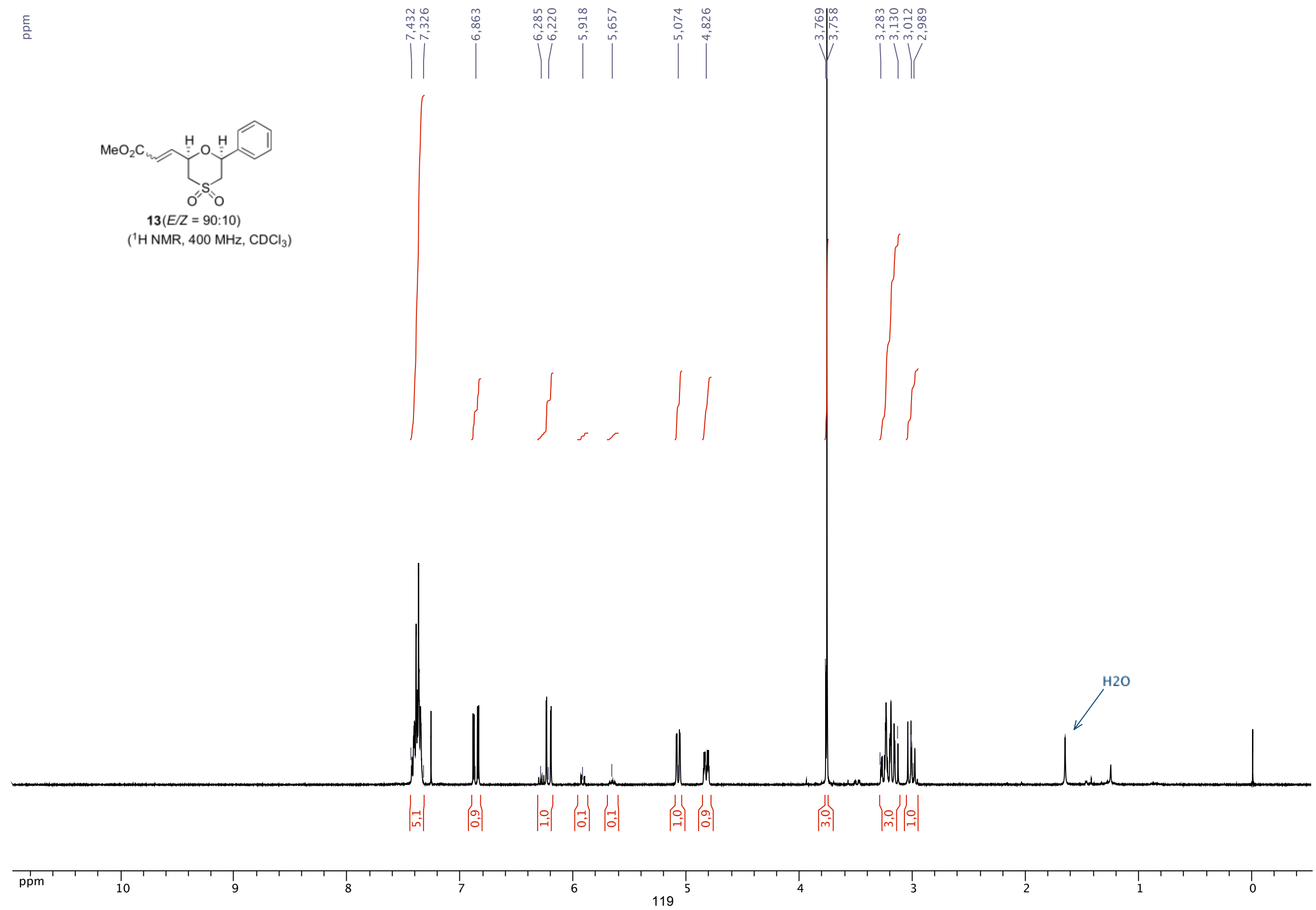

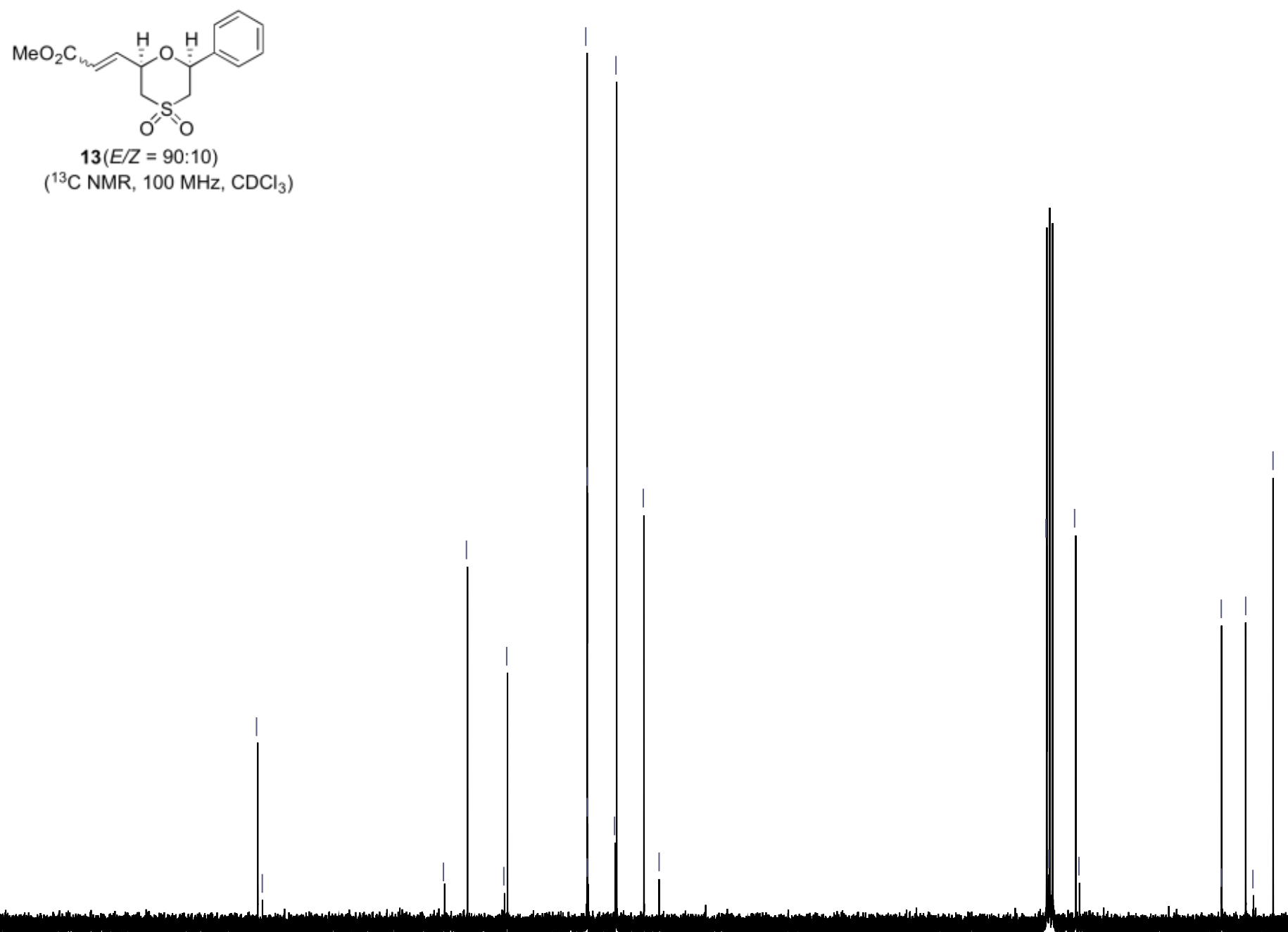

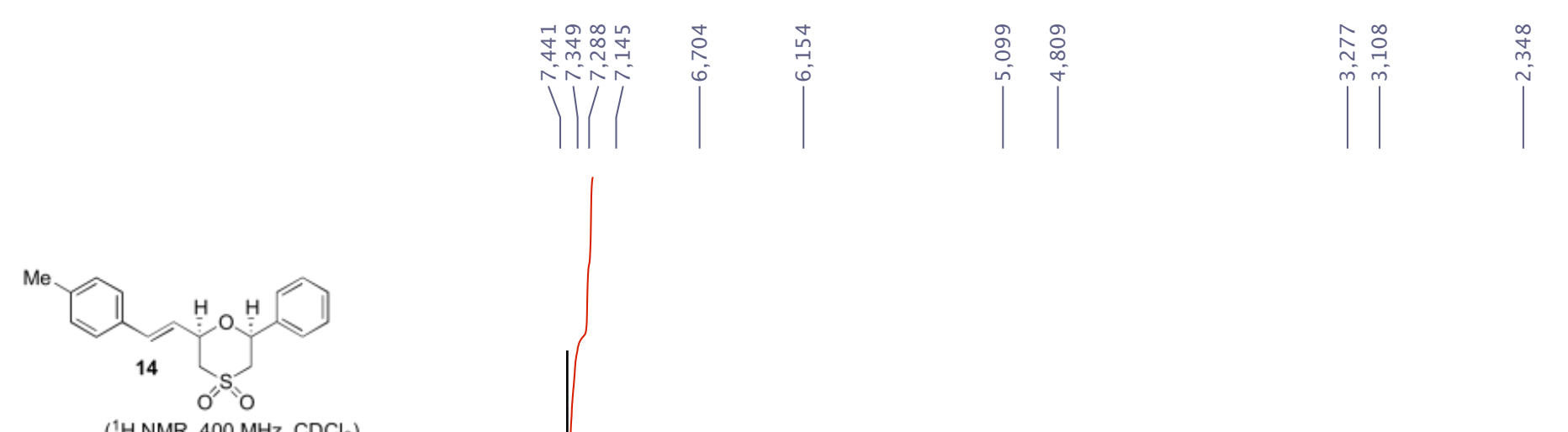

( ${ }^{1} \mathrm{HNMR}, 400 \mathrm{MHz}, \mathrm{CDCl}_{3}$ )

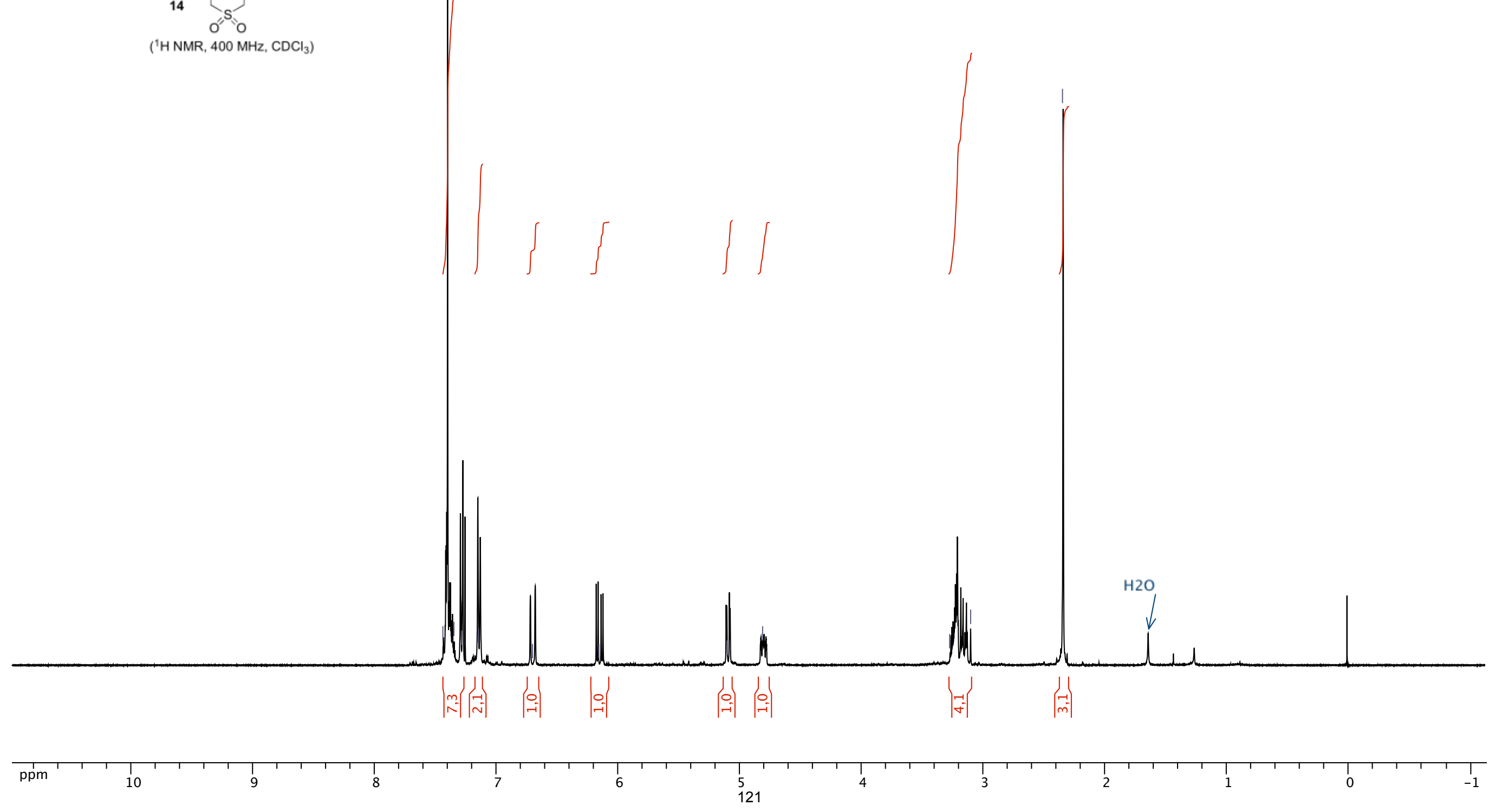


Me

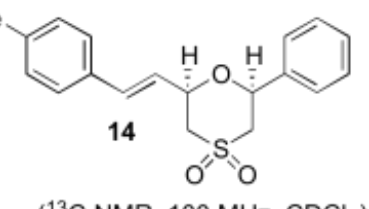

$\left({ }^{13} \mathrm{C} \mathrm{NMR}, 100 \mathrm{MHz}, \mathrm{CDCl}_{3}\right)$
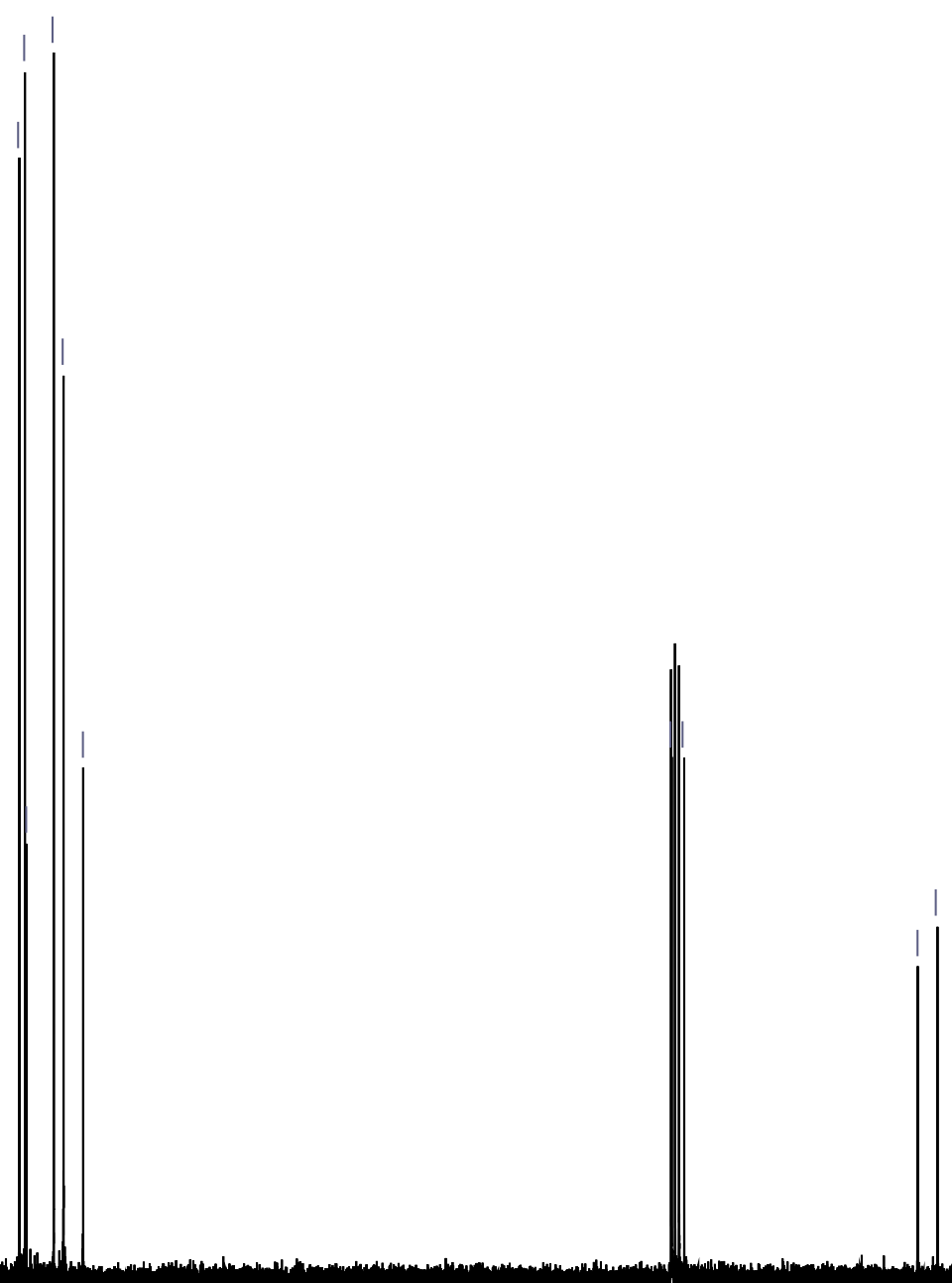

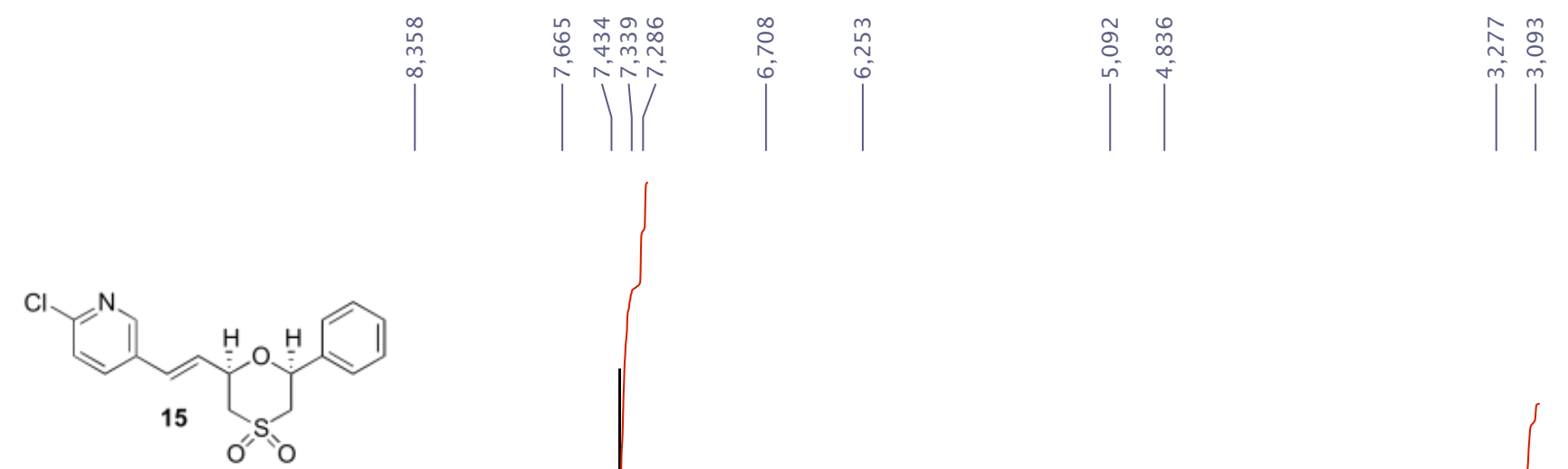

( ${ }^{1} \mathrm{H} \mathrm{NMR}, 400 \mathrm{MHz}, \mathrm{CDCl}_{3}$ )

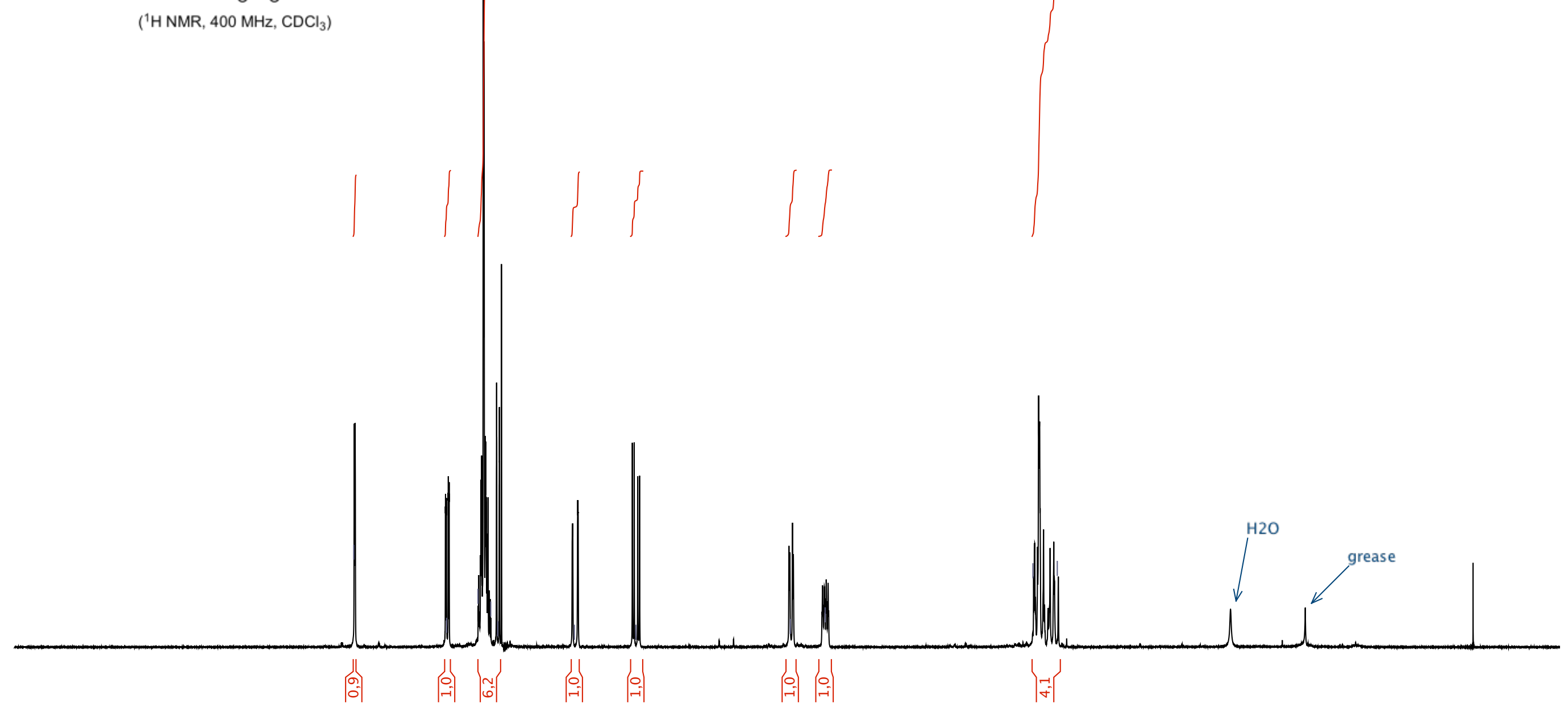



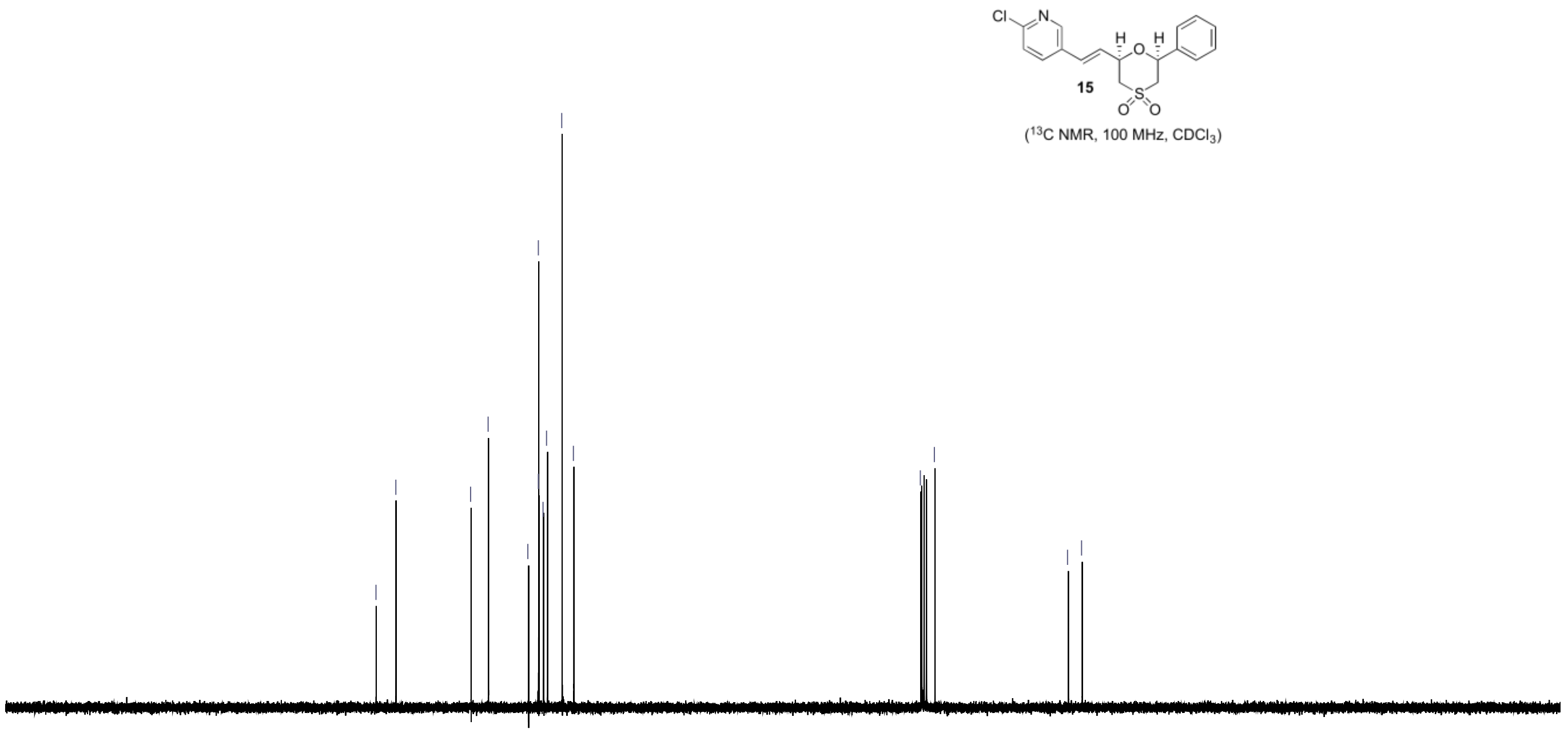


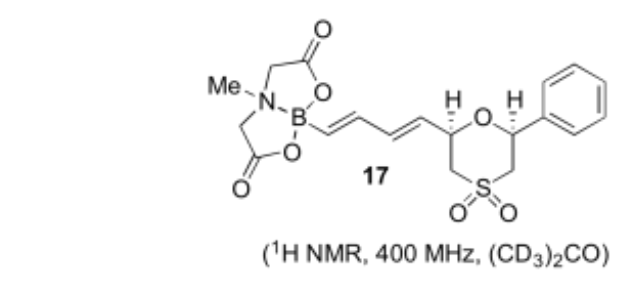

( $\left.{ }^{1} \mathrm{H} \mathrm{NMR}, 400 \mathrm{MHz},\left(\mathrm{CD}_{3}\right)_{2} \mathrm{CO}\right)$
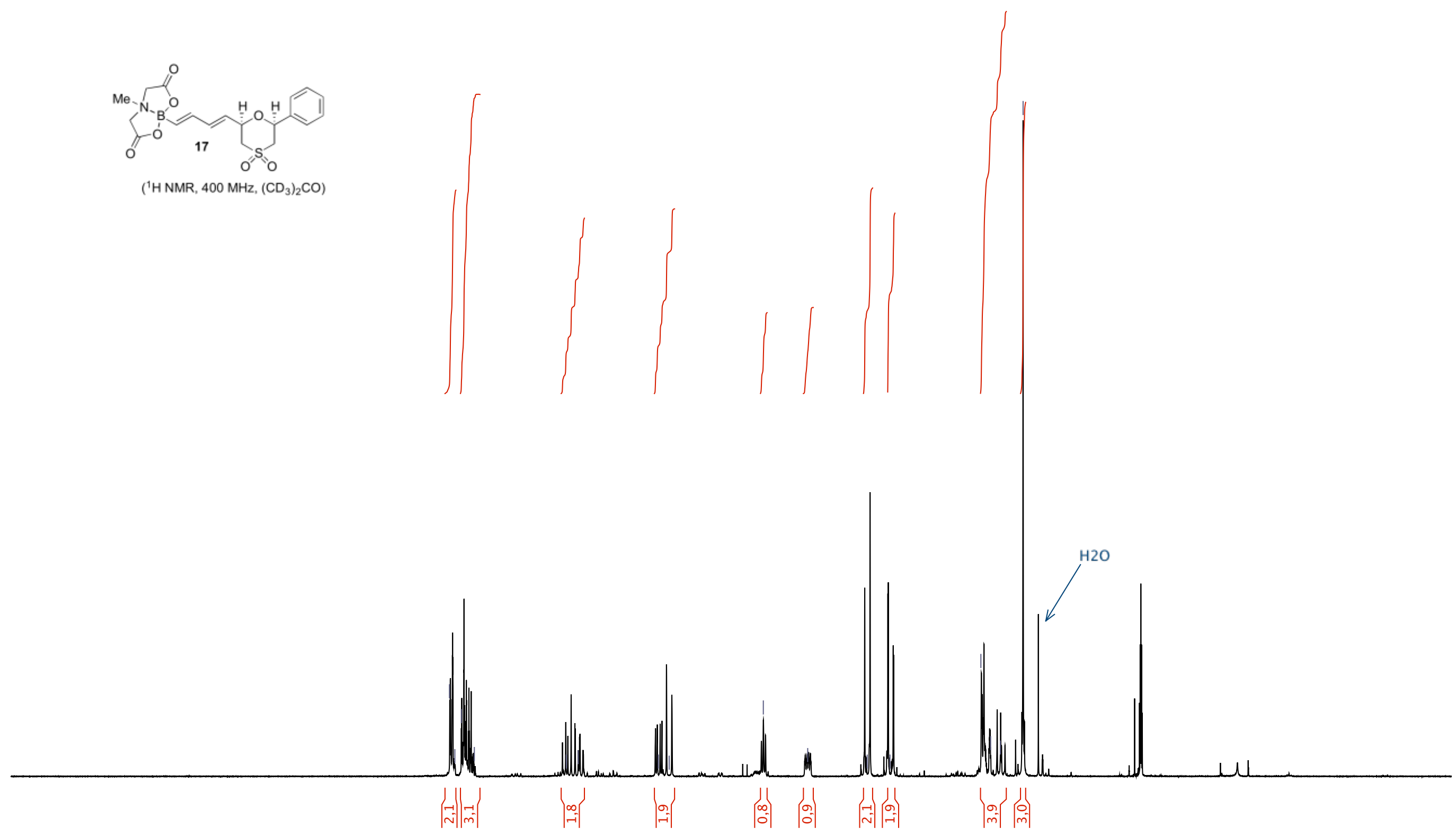

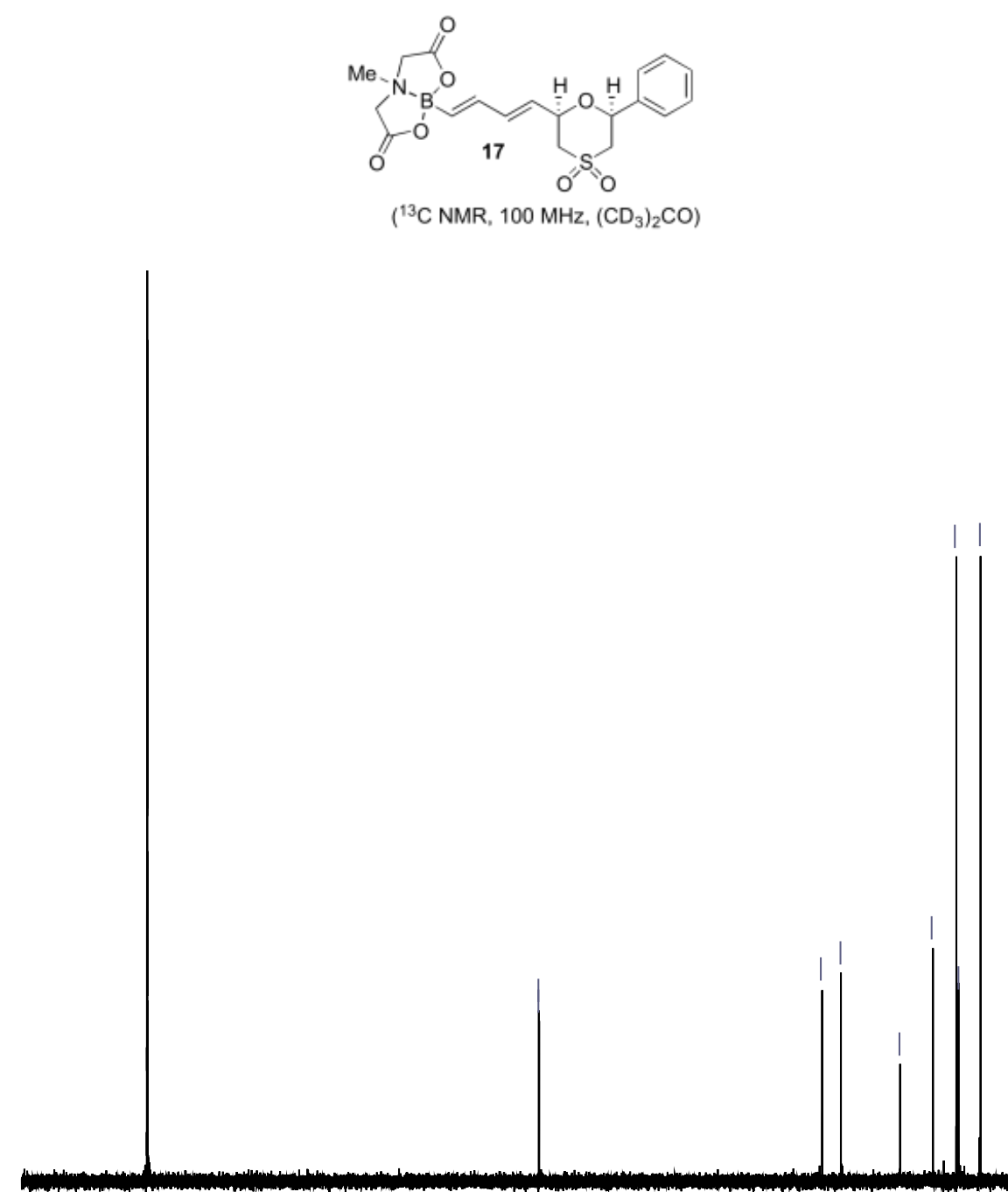